\title{
SPLINES GENERALIZADOS Y SOLUCIÓN NODAL EXACTA EN EL MÉTODO DE ELEMENTOS FINITOS
}

\author{
(GENERALIZED SPLINES AND EXACT NODAL SOLUTION BY FINITE ELEMENT \\ METHOD)
}

José L. Romero, Dr. Ingeniero de Caminos. Facultad de Informática, U. P. M.

Miguel A. Ortega, Ingeniero de Caminos

ESPAÑA

Fecha de recepción: 7-VII-99

$400-22$

\begin{abstract}
RESUMEN
Se desarrolla en este trabajo un método de construcción de splines generalizados que está basado en una interpretación matricial o estructural de la teoría matemática de dichas funciones. Se sugiere, a lo largo del desarrollo realizado,que tanto la terminología como los métodos de análisis en cálculo de estructuras y resistencia de materiales son muy naturales $y$, por tanto, idóneos para este campo de los splines. El método propuesto permite abordar, con una única y sencilla metodología, el tratamiento de tipos de splines muy diferentes:cambio de las caracteristicas del spline de unos subintervalos a otros (modificación de pesos, parámetros de tensión, etc.), diferentes condiciones de interpolación en los distintos nodos, etc. Se destaca como aportación de interés la consideración de nuevas condiciones de interpolación definidas como acciones de tipo puntual (cargas). Asimismo, se interpreta y demuestra que la solución de problemas de contorno unidimensionales por el método de elementos finitos es nodalmente exacta cuando se utilizan ciertos espacios de aproximación de dimensión finita engendrados por splines generalizados. También se desarrolla el concepto de acción equivalente como generalización del de acción nodal equivalente. Finalmente se ilustra la aplicación de la metodología desarrollada, basada en la interpretación matricial citada, con ejemplos de splines en el campo de los gráficos, en el análisis de vigas continuas sobre fundación elástica, sometidas a flexión y tracción o compresión y en problemas dinámicos.
\end{abstract}

\section{SUMMARY}

This paper discusses a method for constructing generalized splines, which is based on a matrix or structural interpretation of the mathematical theory on these functions. It is suggested throughout the paper that both the terminology and the methods of analysis in structures and strength of materials are very natural and, therefore, apt for this field of splines. The proposed method allows a wide range of splines to be addressed by means of a single and simple methodology: changing the characteristics of the spline from some subintervals to others (modification of weights, tension parameters, etc.), different conditions of interpolation in different nodes, etc. A noteworthy contribution is that new conditions of interpolation are considered, which are defined as individual actions (loads). Furthermore, it is found and shown that the solution of one-dimensional boundary value problems using the finite elements method is exact at the nodal points when certain spaces of finite dimension approximation engendered by generalized splines are used. The concept of equivalent action is developed as a generalization of the notion of equivalent nodal action (equivalent nodal loads). Finally, an illustration is given of how the developed methodology, based on the aforesaid matrix interpretation, can be applied, including examples of splines in the field of graphics, analysis of continuous beams on an elastic foundation, subjected to bending moment and tēnsion or compression, and in dynamic problems. 


\section{Introducción}

Las funciones splines (splines) constituyen, desde hace un tiempo, una herramienta de gran interés en la resolución de problemas de ingenieria relacionados con el ajuste y trazado de curvas y superficies y diseño gráfico en general, así como con el cálculo y análisis por elementos finitos ([1] a [20]). Tal y como se indica en las notas históricas del texto de Schumaker ([3]), las funciones splines fueron ya utilizadas en trabajos de Runge en 1901, de Eagle en 1928, de Quade y Collatz en 1938 y Favard en 1940. Sin embargo, en ninguno de ellos se daba ese nombre a dichas funciones, pues éstas eran denominadas, de manera un tanto genérica, como funciones de interpolación osculatoria. El término "spline" , aparece por primera vez en un trabajo de Schoenberg en 1946, en el cual se justifica dicho término por la relación que hay entre la gráfica de ciertas funciones polinómicas a trozos y la deformada del dispositivo mecánico de trazado, utilizado por los delineantes, denominado, en la terminología inglesa, con el nombre de spline. En definitiva, se está haciendo referencia al spline cúbico que, como es conocido, constituye el modelo matemático de la elástica para el caso de acciones puntuales, cuando se aproxima linealmente la curvatura mediante la derivada segunda del desplazamiento.

En el momento actual la teoria de splines se encuentra en una fase de desarrollo muy avanzada, habiendo seguido, en este sentido, un proceso análogo al de la teoría de elementos finitos, pues, en cierto modo, es parte integrante de esta última. Sin embargo, por lo que se puede apreciar en la bibliografia correspondiente, la teoría de splines no ha heredado de la misma forma que los elementos finitos la terminología y, especialmente, la metodología del análisis matricial de estructuras. Asimismo y considerando el caso elemental de los splines cúbicos para el cual la mayor parte de los autores citan la conexión obvia con la teoría de vigas, los métodos de cálculo utilizados rara vez hacen uso, al menos de modo explícito, de las técnicas de cálculo matricial de estructuras, a pesar de la versatilidad y sencillez de éstas.

Desde el punto de vista histórico y limitándonos también al caso de los splines cúbicos, llama la atención la ausencia de citas que hagan referencia a la ecuación de los tres momentos establecidas por Bertot y Clapeyron ([21, p.145]), a mediados del pasado siglo. Dicha ecuación se relaciona trivialmente con cada una de las ecuaciones que permiten determinar el spline cúbico en términos de las derivadas segundas (momentos). Por otra parte, referencias detalladas a los métodos energéticos iniciados por Clapeyron y desarrollados con profundidad por Castigliano en 1875 y 1879 y que equivalen a la propiedad de mínima norma en teoría de splines establecida en el teorema de Holladay en 1957 ([1, p. 3]), son muy limitadas en textos tan conocidos y que documentan con tanto grado de detalle la teoria de splines, como, por ejemplo, los indicados en las referencias siguientes: $[1,2,3,4,5,6]$.

Todo lo anterior viene a señalar que, quizás, no se haya explotado suficientemente la interpretación matricial o estructural de esta teoria, especialmente en lo referente a la construcción, desarrollo y análisis de los diferentes tipos de splines. Una razón para lo anterior podría ser la existencia de técnicas de cálculo de splines basadas en la representación de dichas funciones mediante bases de B-splines ([6]), las cuales, por otra parte, han demostrado ser muy eficientes desde el punto de vista computacional, especialmente en el caso de splines polinómicos.

El propósito fundamental de este artículo es precisamente el de exponer algunos aspectos de la teoría de splines utilizando, como herramienta, técnicas de cálculo inspiradas en el análisis matricial de estructuras. En particular se aborda la construcción de los splines generalizados, considerando dichas funciones como soluciones, en el sentido de las distribuciones, de ciertos problemas de contorno autoadjuntos, para los que el término independiente de la ecuación diferencial viene definido por funciones que representan, en términos físicos, acciones de tipo puntual definidas mediante deltas de Dirac y sus derivadas. Se obtiene la ecuación matricial del spline con formulaciones en desplazamientos y se comentan, asimismo, algunos aspectos del método de compatibilidad en relación con el cálculo de splines.

Entre las diferentes consecuencias de la formulación matricial que se propone, señalamos en primer lugar el hecho de abordar, con una única y sencilla metodologia el tratamiento de muy diferentes tipos de splines. En segundo lugar y como resultado de interés destacamos que la construcción matricial de los splines generalizados, conduce, de manera natural, a un cálculo por elementos finitos que permite obtener soluciones nodalmente exactas en ciertos problemas de contorno, obteniendo, en este sentido, los mismos resultados que $P$. Tong ([22]), aunque desde un planteamiento inicial diferente. En este último aspecto, la metodologia que se propone guarda gran relación con el método de Trefft ([23]), y con lo que podría ser una versión matricial de la función de Green o método de los elementos de contorno en dimensión uno. Asimismo el planteamiento seguido nos ha conducido al concepto de acción (repartida) equivalente. Por otra parte hay que indicar que muchos de los aspectos que se tratan en este trabajo con la metodologia matricial propuesta, han sido estudiados extensamente por otras vias, en diversos artículos sobre splines generalizados y problemas de contorno autoadjuntos para n puntos $([24,25,26])$. Sin embargo, el carácter sistemático del enfoque matricial que proponemos, además de las interpretaciones que de manera directa permite establecer, consideramos que puede tener ciertas ventajas respecto a otros desarrollos 
condiciones relativas a acciones puntuales. Estas posibilidades se ilustran al final del trabajo con aplicaciones en el campo de los gráficos, en análisis de vigas sobre fundación elástica y en problemas dinámicos.

Finalmente indicamos que en anteriores publicaciones $[27,28,29]$ se aborda, con un planteamiento diferente al del presente trabajo, el cálculo de splines generalizados mediante técnicas de relajación inspiradas en los métodos de Cross y Kani de cálculo de estructuras, proporcionando algoritmos, que minimizan cierto funcional cuadrático de tipo eliptico relativo a la energia de deformación del spline y que permiten obtener, en la práctica y de manera sencilla, no sólo splines de grado impar arbitrario sino tambièn cierta clase de splines generalizados. En dichas referencias está patente la analogía estructural, aunque los métodos que alli se plantean están orientados desde el inicio hacia un cálculo de tipo iterativo de los splines.

\section{Preliminares}

Se exponen a continuación algunas propiedades de los operadores diferenciales de orden $m$ y $2 m$, que son utilizadas posteriormente. Dichas propiedades son consecuencia, de una manera más o menos directa, de la fórmula de integración por partes.

Sea el operador diferencial $L$ de orden $m(m \geq 1)$ definido en el espacio de funciones $C^{m}[a, b]$

$$
L(u)=a_{m} D^{m} u+a_{m-1} D^{m-1} u+. .+a_{1} D u+a_{0} u
$$

donde para $0 \leq j \leq m, D^{j} \cdot \equiv\left(\frac{d^{j} \cdot}{d x^{j}}\right) \equiv(\cdot)^{(j)}$, y $a_{j}=a_{j}(x) \in C^{j}[a, b]$, con $a_{m} \geq \delta>0$, para todo $x \in[a, b]$. Integrando por partes el producto, $L(u) v$, en el intervalo $[a, b]$, donde $v$ es una función genérica del espacio $C^{m}[a, b]$, se obtiene ([30, p. 211], [31, p. 168]) la relación conocida como fórmula de Green:

$$
\int_{a}^{b} L(u) v d x=\left.P(u, v)\right|_{a} ^{b}+\int_{a}^{b} L^{*}(v) u d x
$$

que es equivalente a la siguiente expresión, en forma diferencial, conocida como identidad de Lagrange

$$
L(u) v=\frac{d}{d x}\{P(u, v)\}+L^{*}(v) u
$$

donde $L^{*}$ es el operador adjunto (adjunto formal) de $L$ y $P$ es la llamada concomitante bilineal. Las expresiones de $L^{*}(v)$ y $P(u, v)$ son respectivamente

$$
\begin{gathered}
L^{*}(v)=\sum_{j=0}^{m}(-1)^{j} D^{j}\left(a_{j} v\right)= \\
=(-1)^{m} \cdot D^{m}\left(a_{m} v\right)+(-1)^{m-1} D^{m-1}\left(a_{m-1} v\right)+. .+(-1) D\left(a_{1} v\right)+a_{0} v \\
P(u, v)=\sum_{i=0}^{m-1} u^{(i)} p_{i}(v)
\end{gathered}
$$

donde

$$
\begin{aligned}
& p_{0}(v)=(-1)^{m-1} D^{m-1}\left(a_{m} v\right)+(-1)^{m-2} D^{m-2}\left(a_{m-1} v\right)+\ldots+(-1) D\left(a_{2} v\right)+a_{1} v \\
& p_{1}(v)=(-1)^{m-2} D^{m-2}\left(a_{m} v\right)+(-1)^{m-3} D^{m-3}\left(a_{m-1} v\right)+\ldots+(-1) D\left(a_{3} v\right)+a_{2} v \\
& \quad \vdots \\
& p_{m-2}(v)=(-1) D\left(a_{m} v\right)+a_{m-1} v \\
& p_{m-1}(v)=a_{m} v
\end{aligned}
$$

es decir, los operadores $p_{i}$ vienen definidos por la expresión

$$
p_{i}(v)=\sum_{j=0}^{m-1-i}(-1)^{j} D^{j}\left(a_{j+1+i} v\right)
$$


tal y como se deduce, de manera sencilla, mediante el proceso de integración por partes indicado. Es interesante, por otra parte, interpretar los operadores diferenciales $p_{i}$ como adjuntos de otros operadores de orden $m-1-i$.

En efecto, obsérvese que llamando $r_{i}(v)=\sum_{j=0}^{m-1-i} a_{j+1+i} D^{j} v$ se tiene que $p_{i}=r_{i}^{*}$ y la expresión de la concomitante puede ponerse como

$$
P(u, v)=\sum_{i=0}^{m-1} u^{(i)} r_{i}^{*}(v)
$$

La expresión (2.2) de la fórmula de Green puede ponerse en la forma

$$
\int_{a}^{b} L(u) v d x=\sum_{i=1}^{2 m} l_{i}(u) \bar{l}_{i}(v)+\int_{a}^{b} L^{*}(v) u d x
$$

donde $l_{i}(u)$ y $\bar{l}_{i}(v)$ son las formas lineales siguientes

$$
\begin{aligned}
& l_{k}(u)=u^{(k-1)}(a), \quad l_{k+m}(u)=u^{(k-1)}(b) \\
& \bar{l}_{k}(v)=-p_{k-1}(v)_{x=a}, \quad \bar{l}_{k+m}(v)=p_{k-1}(v)_{x=b} \\
& k=1, . ., m_{l}
\end{aligned}
$$

y son las que en la terminologia de elementos finitos y elementos de contorno generan respectivamente las llamadas condiciones de contorno esenciales y naturales ([32, p. 117], [33, p. 15]).

Propiedades de interés, para nuestros propósitos, de los operadores diferenciales $L$ y $L^{*}$ son las siguientes ([30, pp. 126,131]:

(I) El operador adjunto de $L^{*}$ es $L$, es decir $\left(L^{*}\right)^{*}=L$. Asimismo el adjunto de la composición de dos operadores es la composición de los adjuntos en orden contrario, es decir $\left(L_{1} L_{2}\right)^{*}=L_{2}^{*} L_{1}^{*}$

Cuando un operador diferencial coincide con su adjunto se dice que es autoadjunto. Se tienen para este caso y limitándonos a orden par, los siguientes resultados:

\section{( II) Todo operador diferencial $L$ autoadjunto de orden $2 m$ puede ponerse en la forma} $L(u)=\sum_{j=0}^{m}(-1)^{j} D^{j}\left(a_{j} D^{j} u\right)$. Asimismo el citado operador L puede también ser expresado en forma factorizada como $L=L_{1}^{*} L_{1}$ donde $L_{1}$ es un operador de orden $m$.

Veamos a continuación una aplicación de la fórmula de Green al caso donde el operador diferencial $L$ es autoadjunto.

Consideremos en primer lugar el caso donde el operador está en la forma autoadjunta usual

$$
L(u)=\sum_{j=0}^{m}(-1)^{j} D^{j}\left(a_{j} D^{j} u\right)
$$

Integrando por partes el producto $L(u) v$ en el intervalo $[a, b]$, donde $v$ es una función arbitraria de la clase $C^{2 m}$ en el intervalo citado, resulta

$$
\int_{a}^{b} L(u) v d x=\left.\left((-1) \sum_{i=0}^{m-1} v^{(i)} P_{i}(u)\right)\right|_{a} ^{b}+a(u, v)
$$

donde, de manera análoga a (2.3), los operadores $P_{i}$ están definidos como

$$
\begin{aligned}
& P_{0}(u)=(-1)^{m-1} D^{m-1}\left(a_{m} D^{m} u\right)+(-1)^{m-2} D^{m-2}\left(a_{m-1} D^{m-1} u\right)+. .+a_{1} D u \\
& P_{1}(u)=(-1)^{m-2} D^{m-2}\left(a_{m} D^{m} u\right)+(-1)^{m-3} D^{m-3}\left(a_{m-1} D^{m-1} u\right)+. .+a_{2} D^{2} u=
\end{aligned}
$$




$$
\begin{aligned}
& P_{m-3}(u)=(-1)^{2} D^{2}\left(a_{m} D^{m} u\right)+(-1) D\left(a_{m-1} D^{m-1} u\right)+a_{m-2} D^{m-2} u \\
& P_{m-2}(u)=(-1) D\left(a_{m} D^{m} u\right)+a_{m-1} D^{m-1} u \\
& P_{m-1}(u)=a_{m} D^{m} u
\end{aligned}
$$

y $a(u, v)$ es la forma bilineal simétrica definida como

$$
a(u, v)=\int_{a}^{b}\left(\sum_{j=0}^{m} a_{j} D^{j} u D^{j} v\right) d x
$$

La expresión (2.6) puede ponerse también en la forma siguiente

$$
\int_{b}^{b} L(u) v d x=-\sum_{i=1}^{2 m} l_{i}(v) \bar{l}_{i}(u)+\int_{i}^{b} \sum_{j=0}^{m} a_{j} D^{j} u D^{j} v d x
$$

que se reduce a esta otra, integrando por partes el segundo sumando del segundo miembro

$$
\int_{a}^{b} L(u) v d x=\sum_{i=1}^{2 m} l_{i}(u) \bar{l}_{i}(v)-\sum_{i=1}^{2 m} l_{i}(v) \bar{l}_{i}(u)+\int_{b}^{b} L(v) u d x
$$

donde para (2.9) y (2.10) las formas lineales $l_{i}$ son las mismas que en (2.4), sin embargo las $\bar{l}_{i}$ están definidas ahora por

$$
\begin{gathered}
\bar{l}_{k}(u)=-P_{k-1}(u)_{x=a}, \quad \bar{l}_{k+m}(u)=P_{k-1}(u)_{x=b} \\
k=1, . ., m
\end{gathered}
$$

Obsérvese que cuando en (2.10) $u$ y $v$ son funciones pertenecientes al núcleo del operador $L$ resulta la siguiente expresión de reciprocidad

$$
\sum_{i=1}^{2 m} l_{i}(u) \bar{l}_{i}(v)=\sum_{i=1}^{2 m} l_{i}(v) \bar{l}_{i}(u)
$$

Por otra parte, en el caso de que $u$ y $v$ verifiquen en $[a, b], L(u)=f, L(v)=g$ se tiene de (2.9)

$$
\begin{aligned}
a(u, v)-\sum_{i=1}^{2 m} l_{i}(v) \bar{l}_{i}(u)= & \int_{a}^{b} f v d x, a(v, u)-\sum_{i=1}^{2 m} l_{i}(u) \bar{l}_{i}(v)=\int_{b}^{b} g u d x, \text { y de la simetría de } a(u, v) \\
& \sum_{i=1}^{2 m} l_{i}(v) \bar{l}_{i}(u)+\int_{d}^{b} f v d x=\sum_{i=1}^{2 m} l_{i}(u) \bar{l}_{i}(v)+\int_{a}^{b} g u d x
\end{aligned}
$$

En un apartado posterior, se interpretarán las relaciónes anteriores (2.12) y (2.13), en términos del teorema de reciprocidad ([34, p. 149], [21, pp. 207 y 320]).

Finalmente para el caso donde el operador diferencial viene dado en la forma autoadjunta factorizada

$$
L(u)=L_{1}^{*} L_{1}(u)
$$

con $L_{1}$ de la forma indicada en (2.1) y $a_{j} \in C^{m}[a, b], 0 \leq j \leq m$, integrando por partes el producto $L(u) v$, donde $v$ es una función arbitraria de la clase $C^{2 m}$ en dicho intervalo, aplicando (2.2) y llamando $w=L_{1}(u)$ resulta

$$
\begin{gathered}
\int_{a}^{b} L_{1}^{*} L_{1}(u) v d x=\int_{a}^{b} L_{1}^{*}(w) v d x=-\left.P(v, w)\right|_{a} ^{b}+\int_{a}^{b} L_{1}(v) w d x= \\
=-\left.P\left(v, L_{1}(u)\right)\right|_{a} ^{b}+a_{1}(u, v)
\end{gathered}
$$

donde la forma bilineal simétrica $a_{1}(u, v)$ está definida por

De manera análoga también se tiene

$$
a_{1}(u, v)=\int_{a}^{b} L_{1}(u) L_{1}(v) d x
$$

$$
\int_{a}^{b} L_{1}^{*} L_{1}(v) u d x=-\left.P\left(u, L_{1}(v)\right)\right|_{a} ^{b}+\int_{a}^{b} L_{1}(v) L_{1}(u) d x
$$


resultando de las dos expresiones anteriores que

$$
\int_{a}^{b} L_{1}^{*} L_{1}(u) v d x=-\left.P\left(v, L_{1}(u)\right)\right|_{a} ^{b}+\left.P\left(u, L_{1}(v)\right)\right|_{a} ^{b}+\int_{a}^{b} L_{1}^{*} L_{1}(v) u d x
$$

Análogamente al caso anterior, las expresiones (2.15) y (2.16) pueden ponerse de la siguiente forma

$$
\begin{gathered}
\int_{a}^{b} L_{1}^{*} L_{1}(u) v d x=-\sum_{i=1}^{2 m} l_{i}(v) \bar{l}_{i}\left(L_{1}(u)\right)+\int_{a}^{b} L_{1}(v) L_{1}(u) d x \\
\int_{a}^{b} L_{1}^{*} L_{1}(u) v d x=\sum_{i=1}^{2 m} l_{i}(u) \bar{l}_{i}\left(L_{1}(v)\right)-\sum_{i=1}^{2 m} l_{i}(v) \bar{l}_{i}\left(L_{1}(u)\right)+\int_{a}^{b} L_{1}^{*} L_{1}(v) u d x
\end{gathered}
$$

En este caso las formas lineales $l_{i}$ y $\bar{l}_{i}$ vienen definidas por las expresiones dadas en (2.4).

En las mismas condiciones que antes para $u$ y $v$ se tienen los resultado de reciprocidad análogos a (2.12) y (2.13)

$$
\begin{gathered}
\sum_{i=1}^{2 m} l_{i}(u) \bar{l}_{i}\left(L_{1}(v)\right)=\sum_{i=1}^{2 m} l_{i}(v) \bar{l}_{i}\left(L_{1}(u)\right) \\
\sum_{i=1}^{2 m} l_{i}(u) \bar{l}_{i}\left(L_{1}(v)\right)+\int_{a}^{b} g u d x=\sum_{i=1}^{2 m} l_{i}(v) \bar{l}_{i}\left(L_{1}(u)\right)+\int_{a}^{b} f v d x
\end{gathered}
$$

\section{Sobre el concepto de spline generalizado}

Como es bien conocido, un spline es una función definida a trozos sobre subintervalos con cierto grado de regularidad, esto es, se tiene continuidad de la función y de las derivadas hasta las de un orden determinado. El tipo de spline más extendido es el spline ordinario o función spline de grado $k$, definido por polinomios de grado menor o igual que $k$ en cada subintervalo, de modo que la función es continua y con derivadas continuas hasta la de orden $k-1$. Para los splines generalizados, cuya definición precisa se da después, la función a trozos viene definida por funciones en general no polinómicas, que son soluciones en cada subintervalo de una ecuación diferencial homogénea.

La construcción de los splines polinómicos de grado $k$ para $k$ pequeño (menor o igual que tres) es sencilla y puede abordarse de manera directa a partir de la definición. Para el caso cúbico conviene, como se sabe, emplear como incógnitas, en lugar de los coeficientes de los polinomios cúbicos de los distintos subintervalos, bien a las derivadas primeras o bien a las derivadas segundas de la función en los nodos de separación de los subintervalos. Esta elección de incógnitas auxiliares no es fortuita, pues, además de reducir el número de incógnitas a aproximadamente la cuarta parte, tiene la ventaja de poder realizar una interpretación estructural de las mismas en términos de giros y momentos flectores de una cierta elástica.

Para splines de grado superior, la construcción directa de tales funciones a partir de la definición, mediante la simple manipulación algebráica, no resulta tan inmediata como en los casos citados, incluso la obtención de las propiedades fundamentales puede resultar un proceso complejo y artificioso. Véase, a modo de ejemplo, la construcción y estudio de propiedades de los splines de quinto grado, realizado en [1, p. 143], [35], [36] y [7, p. 181]. Por otra parte, hay que decir que la determinación de splines mediante bases de B-splines ([6]) y, en particular, la construcción de dichas bases mediante la fórmula de recurrencia de Cox-de Boor ha facilitado, en gran medida, la obtención de funciones splines, especialmente en el caso de splines polinómicos. Aun así se puede apreciar en los textos más conocidos ([1, 2, 3, 5, 6]) y en la bibliografia consultada, la ausencia explícita de ciertos aspectos, que permitirian un tratamiento más unificado y sistemático de la teoría de splines. Nos referimos a una interpretación matricial o estructural de los splines. Dicho enfoque resulta, tal y como se ha señalado en la introducción, muy natural si consideramos el origen de la teoria de splines y la proximidad de ésta con la de elementos finitos $y$, particularmente, con la de elementos de contorno en dimensión uno.

De una manera esquemática, la teoria de splines, tal y como se indica en [1] puede abordarse desde dos puntos de vista: el algebraico y el variacional. El primero se basa en construir, a partir de la definición y mediante manipulaciones algebraicas, el sistema que define al spline y el segundo punto de vista se basa en realizar un estudio previo de propiedades de tipo intrínseco o variacional conocidas como relaciones integrales. En el caso de splines polinómicos, el segundo enfoque suele restringirse a los splines de grado impar.

Nuestro planteamiento en este trabajo se basa, fundamentalmente, en el segundo punto de vista y se realiza para los denominados splines generalizados o L-splines ([1, p. 191], [37]), los cuales incluyen como caso particular a los splines polinómicos usuales. 
A continuación pasamos a definir los splines generalizados, relajando, algo más de lo que es habitual, los requerimientos de regularidad.

Consideremos el intervalo $\left[x_{1}, x_{n}\right]$ descompuesto en la unión de subintervalos $\left[x_{i}, x_{i+1}\right]$, donde $i=1, . ., n-2$, es decir

$$
\left[x_{1}, x_{n}\right]=\bigcup_{i=1}^{n-1}\left[x_{i}, x_{i+1}\right]
$$

donde los nodos $x_{i}$ verifican la condición

$$
x_{1}<x_{2}<. .<x_{i}<x_{i+1}<. .<x_{n}
$$

y sea $L$ el operador diferencial autoadjunto de orden $2 m$ dado en (2.5) o equivalentemente el dado en (2.14), donde cada subintervalo $\left[x_{i}, x_{i+1}\right]$ es un caso particular del intervalo de extremos $a y$ $b$ considerado en el apartado anterior.

Con las condiciones anteriores, denominamos spline generalizado de orden $2 \mathrm{~m}$ o $L$-spline a toda función $u(x)$ de la clase $C^{m-1}\left[x_{1}, x_{n}\right]$ que satisface la ecuación diferencial $L(u(x))=0$ en cada subintervalo abierto $\left(x_{i}, x_{i+1}\right)$, es decir

$$
u \in C^{m-1}\left[x_{1}, x_{n}\right] \quad L(u)=0, \quad x \in \bigcup_{i=1}^{n-1}\left(x_{i}, x_{i+1}\right)
$$

Obsérvese de la definición que la función $u$ es continua y con tcdas las derivadas continuas, al menos, hasta la de orden $m-1$ en todo el intervalo $\left[x_{1}, x_{n}\right]$, sin embargo $u$ en cada subintervalo abierto $\left(x_{i}, x_{i+1}\right)$ es de la clase $C^{2 m}$. Como puede verse un spline generalizado es una función definida a trozos formada por elementos de $N(L)$ (núcleo del operador $L$ ) en cada subintervalo. Por otra parte, al contrario de lo que es usual, las condiciones de regularidad de los coeficientes que definen al operador $L$, las referimos aqui a cada subintervalo $\left(x_{i}, x_{i+1}\right), i=1, . ., n-1$, es decir

$$
a_{j}=a_{j}(x) \in C^{j}\left(\bigcup_{i=1}^{n-1}\left(x_{i}, x_{i+1}\right)\right), j=0, . ., m
$$

con $a_{m} \geq \delta>0$ para todo $x \in \bigcup_{i=1}^{n-1}\left(x_{i}, x_{i+1}\right)$ (para el caso de operador en la forma factorizada $\left.a_{j}=a_{j}(x) \in C^{m}\left(\bigcup_{i=1}^{n-1}\left(x_{i}, x_{i+1}\right)\right)\right)$, aunque se exige al mismo tiempo que los coeficientes deben verificar condiciones de regularidad adicionales como la existencia y finitud en cada subintervalo de los limites siguientes: $\lim _{x \rightarrow c} a_{j}^{(k)}(x), c=x_{i}^{+}, x_{i+1}^{-}, k=0, . ., j$ con objeto de garantizar la existencia y finitud también de los límites $u^{(k)}\left(x_{i}^{+}\right)=\lim _{x \rightarrow x_{i}^{+}} u^{(k)}(x), u^{(k)}\left(x_{i+1}^{-}\right)=\lim _{x \rightarrow x_{i+1}^{-}} u^{(k)}(x), k=0, . ., 2 m$ y

$$
P_{k}(u)_{x=x_{i}^{+}}=\lim _{x \rightarrow x_{i}^{+}} P_{k}(u(x)), P_{k}(u)_{x=x_{i+1}^{-}}=\lim _{x \rightarrow x_{i+1}^{-}} P_{k}(u(x))
$$

NOTA: De esta manera los resultados que se han dado en el apartado de preliminares para el intervalo cerrado $[a, b]$, se pueden extender al intervalo abierto génerico $\left(x_{i}, x_{i+1}\right)$. En lo que sigue no se precisará en exceso esta cuestión y pondremos algunas veces $P_{k}(u)_{x=x_{i}}$ y $P_{k}(u)_{x=x_{i+1}}$ en lugar de $P_{k}(u)_{x=x_{i}^{+}}$y $P_{k}(u)_{x=x_{i+1}^{-}}$por sobreentenderse el significado.

Esta relajación parcial de las condiciones de regularidad, es decir, el no exigir que $a_{j}(x) \in C^{j}\left[\varkappa_{1}, x_{n}\right]$, nos va a permitir abordar, desde un enfoque generalista y sistemático, al tiempo que elemental, casos particulares de la teoria, tales como, por ejemplo, los splines con peso y splines en tensión. Este tipo particular de splines ha sido, por otra parte, objeto de estudio en diversos artículos [39, 40,41, 43, 44, 45, 
46], pero en todos ellos el planteamiento seguido ha sido en su mayor parte algebraico y orientado de entrada a la resolución de los casos concretos que se han planteado sus autores.

Cuando las funciones $a_{j}(x)$ que definen el operador diferencial $L$, verifican la condición de ser de la clase $C^{j}$ en todo el intervalo $\left[x_{1}, x_{n}\right]$, se introduce el concepto de deficiencia $z=\left(z_{2}, z_{3}, . ., z_{n-1}\right)$, donde los valores $z_{2}, z_{3}, . ., z_{n-1}$ son enteros positivos tales que $1 \leq z_{i} \leq m$, y viene a ser una medida de la limitación del spline generalizado para satisfacer $L(u(x))=0$ en $\left[x_{1}, x_{n}\right]$, pues la deficiencia $z_{i}$ indica que en los nodos internos $x_{i}, i=2, . ., n-1$, las sucesivas derivadas por la izquierda $y$ derecha son iguales hasta la de orden $2 m-1-z_{i}$, es decir

$$
\begin{aligned}
& u^{(k)}\left(x_{i}^{-}\right)=u^{(k)}\left(x_{i}^{+}\right), \quad 0 \leq k \leq 2 m-1-z_{i} \\
& i=2, . ., n-1
\end{aligned}
$$

Asi el spline ordinario (deficiencia $z_{i}=1, i=2, . ., n-1$ ) es una función de la clase $C^{2 m-2}$ en $\left[x_{1}, x_{n}\right]$ ya que en los nodos interiores puede haber discontinuidad en la derivada de orden $2 m-1$. Por otra parte cuando la deficiencia es máxima $\left(z_{i}=m, i=2, . ., n-1\right)$, los splines son funciones de Hermite (no polinómicas en general) a trozos. Los caso anteriores, cuando el operador diferencial $L$ es de la forma particular $L=(-1)^{m} D^{2 m}$ se reducen respectivamente, a los splines polinómicos ordinarios de grado $2 m-1$ y a los polinomios de Hermite a trozos de grado $2 m-1$.

Es importante señalar que los splines genaralizados no siempre existen para todo operador $L$ sobre cualquier mallado de nodos $x_{i}, i=1, . ., n$ ([1, p.196]). La razón para tal situación se debe a que no siempre es posible obtener a partir de un sistema fundamental de soluciones de la ecuación diferencial homogénea $L(u)=0$ una base de Lagrange en cada subintervalo $\left[x_{i}, x_{i+1}\right] i=1, . ., n-1$ para resolver el problema de interpolación de Hermite generalizado relativo a los datos

$$
u^{(k)}\left(x_{i}\right), u^{(k)}\left(x_{i+1}\right), k=0, . ., m-1 \text {. }
$$

Un ejemplo de lo anterior se tiene para el operador autoadjunto $L u=u^{\prime \prime}+u$ en el intervalo $[0,2 \pi]$, donde del sistema fundamental de soluciones $\{\operatorname{sen} x, \cos x\}$ no puede obtenerse, como es sencillo de verificar, una base de funciones $\left\{N_{1}(x), N_{2}(x)\right.$ tal que

$$
N_{1}(0)=1, N_{1}(2 \pi)=0, N_{2}(0)=0, N_{2}(2 \pi)=1 \text {. }
$$

Esta situación queda resuelta sin embargo para el mismo operador si se toma cualquier intervalo de longitud menor que $\pi$. Aunque no es propósito de este trabajo el entrar en detalles sobre las condiciones que aseguran, en el caso general, la existencia de una base de Lagrange para la interpolación de Hermite indicada, construida a partir de una base de $N(L)$, se da, al menos, el siguiente resultado, que puede derivarse de la teoria de sistemas de Tschebyscheff ([3,p. 423])

( III ) Para el operador $L$ definido en (2.5) o en (2.14) relativo al intervalo [a,b] existe una constante $\rho>0$ tal que para cualquier subintervalo $\left(a_{1}, b_{1}\right)$ de $[a, b]$ de longitud menor que $\rho$ existe la correspondiente base de Lagrange $\left\{N_{i}, i=1, . ., 2 m\right.$, formada por elementos de $N(L)$, para la interpolación definida por las formas lineales de tipo Hermite $l_{i}, i=1, . ., 2 m$, relativas a los extremos del subintervalo

$$
l_{k}(u)=u^{(k-1)}\left(a_{1}\right), l_{k+m}(u)=u^{(k-1)}\left(b_{1}\right), k=1, . ., m
$$

donde $l_{i}\left(N_{j}\right)=\delta_{i j}, i, j=1, . ., 2 m$

A modo de ilustración, se comentan a continuación diversos casos particulares de operadores diferenciales que pueden estudiarse dentro de la teoría de splines generalizados, coma ejemplos elementales de cierto interés práctico. 
1) $L(u)=-\left(A(x) u^{\prime}\right)^{\prime}, \quad$ 2) $L(u)=-\left(A(x) u^{\prime}\right)^{\prime}+B(x) u$, 3) $L(u)=\left(A(x) u^{\prime \prime}\right)^{\prime \prime}$

4) $L(u)=\left(A(x) u^{\prime \prime}\right) "-\left(B(x) u^{\prime}\right)^{\prime}$, 5) $L(u)=\left(A(x) u^{\prime \prime}\right)^{\prime \prime}+C(x) u$

6) $L(u)=\left(A(x) u^{\prime \prime}\right)^{\prime \prime}-\left(B(x) u^{\prime}\right)^{\prime}+C(x) u$

Entre las diferentes posibilidades de interpretación de la función $u$ en términos de diversas magnitudes físicas, nos vamos a limitar a las de carácter mecánico usual. Así, en los dos primeros casos, $u$ puede representar el desplazamiento longitudinal de las secciones de una barra elástica sometida a fuerzas axiles, o bien el desplazamiento transversal de las secciones de un cable sometido a una tracción variable. En el segundo caso, la barra o el cable están embebidos en un medio elástico que ofrece una resistencia proporcional al desplazamiento. El caso tercero es el que corresponde al desplazamiento transversal de una viga de rigidez variable sometida a flexión. Cuando la rigidez $A(x)$ es constante en todo el intervalo $\left[x_{1}, x_{n}\right]$ se tienen los splines cúbicos y cuando la rigidez es constante en cada subintervalo $\left(x_{i}, x_{i+1}\right)$ resultan los splines con peso. Los casos cuarto $y$ quinto corresponden respectivamente al de una viga sometida a flexión y tracción (o compresión si $B(x)<0$ ) y a flexión en un medio elástico (viga flotante con modelo de Winkler). El caso cuarto es denominado, en el campo de los splines, como splines en tensión, splines exponenciales o splines hiperbólicos. Este tipo de splines fue introducido por Schweikert ([38]) con el propósito de reducir el numéro de puntos de inflexión que aparecen algunas veces de forma artificial en problemas de interpolación cuando se emplean los splines cúbicos ordinarios.

Por otra parte el caso sexto corresponde al de una viga sometida a flexión y tracción en un medio elástico de Winkler, apareciendo también en los modelos elaborados por Vlasov y Leontiev para el estudio de vigas en un medio elástico bidimensional de espesor finito ([47]). Este caso, de mayor interés en el campo del análisis estructural que en el de computación gráfica y que incluye además a los tres anteriores como casos particulares, puede ser también analizado empleando la teoria de splines generalizados, lo que permite tratar, en un mismo problema y de manera elemental, situaciones combinadas de los diferentes tipos. Asimismo esta posibilidad de abordar los problemas anteriores desde el punto de vista de la teoría de splines generalizados, permite un enfoque unificado, que aplicado, por ejemplo, al análisis de vigas sobre fundación elástica, contrasta, por su carácter generalista, con el seguido en las referencias [48, 49, $50,51]$.

Finalizamos este punto sobre la definición de los splines generalizados, indicando que la casi totalidad de las publicaciones que tratan el estudio de esta clase de funciones abordan el problema empleando, para el operador diferencial $L$ de orden $2 m$, la forma factorizada $L=L_{1}^{*} L_{1}$ (denominando al spline generalizado como $L_{1}$-spline), sin embargo en nuestro desarrollo y asimismo en las aplicaciones hemos optado por emplear la forma autoadjunta usual indicada en (2.5) por prestarse mejor a las interpretaciones mecánicas del cálculo matricial. Las dos formas de representación del operador conducen, obviamente, a situaciones equivalentes, pero las interpretaciones son diferentes en cada caso tal y como se indica más adelante.

\section{Formulación matricial de los splines generalizados}

Abordamos en este punto lo que hemos venido a denominar como formulación matricial de los splines generalizados. Se trata en definitiva de obtener la ecuación de equilibrio local y, mediante el proceso de ensamblado, la ecuación de equilibrio global del spline generalizado. A partir de dicha ecuación se definen problemas de interpolación con un cierto grado de generalidad, al incluir la posibilidad de considerar también acciones de tipo puntual.

\subsection{Ecuación de equilibrio local del spline generalizado}

Suponiendo que el intervalo genérico $[a, b]$ es de tal longitud que está garantizada la existencia de una base de Lagrange (funciones de forma locales) para la interpolación de Hermite generalizada $\left\{N_{i}, i=1, . ., 2 m\right\}$ relativa a los extremos $a, b$ (véase el resultado $I I I$ ), se tiene con dichas condiciones el siguiente resultado relativo a cada elemento de spline generalizado

( IV ) Sean $L$ el operador diferencial indicado en (2.5), $l_{k}, k=1, . ., 2 m$ las formas lineales definidas en (2.4) y $\bar{l}_{k}, k=1, . ., 2 m$ las definidas en (2.11) y $a(u, v)$ la forma bilineal stmétrica

dada en (2.8). La solución de $L(u)=0$ en $[a, b]$ tal que 


$$
l_{k}(u)=u^{(k-1)}(a)=r_{k}, l_{k+m}(u)=u^{(k-1)}(b)=r_{k+m}, k=1, . ., m
$$

donde $r_{k}, k=1, . ., 2 m$ son números reales arbitrarios dados, verifica la siguiente relación matricial que denominamos ecuación de equilibrio local o ecuación de equilibrio del elemento de spline generalizado en el intervalo $[a, b]$

$$
\widetilde{K} \tilde{u}=\widetilde{q}
$$

donde las matrices $\widetilde{K}=\left(k_{i j}\right)_{i, j=1, \ldots, 2 m}, \tilde{u}$ y $\widetilde{q}$ son

$$
\begin{aligned}
k_{i j} & =k_{j i}=\bar{l}_{i}\left(N_{j}\right)=a\left(N_{i}, N_{j}\right)=\sum_{p=0}^{m} \int_{a}^{b} a_{p}(x)\left(D^{p} N_{i}\right)\left(D^{p} N_{j}\right) d x, i, j=1, . ., 2 m \\
\tilde{u} & =\tilde{r}^{t}=\left(r_{1}, . ., r_{m}, r_{m+1}, . ., r_{2 m}\right) \\
\tilde{q}^{t} & =\left(\bar{l}_{1}(u), . ., \bar{l}_{m}(u), \bar{l}_{m+1}(u), . ., \bar{l}_{2 m}(u)\right)= \\
& =\left(-P_{0}(u)_{x=a}, . .,-P_{m-1}(u)_{x=a}, P_{0}(u)_{x=b}, . ., P_{m-1}(u)_{x=b}\right)
\end{aligned}
$$

con $\widetilde{K}$ simétrica.

En efecto, teniendo en cuenta que $u=\sum_{i=1}^{2 m} r_{i} N_{i}$, ya que $u \in N(L)$, multiplicando ambos miembros de la expresión $L u=0$ por $v=N_{j}, j=1, . ., 2 m$ (por lo que $L v=0$ ), e integrando en $[a, b]$ y aplicando (2.9) resulta

$$
0=-\sum_{i=1}^{2 m} l_{i}\left(N_{j}\right) \bar{l}_{i}(u)+\sum_{i=1}^{2 m} r_{i}\left(\sum_{p=0}^{m} \int_{i}^{b} a_{p} N_{i}^{(p)} N_{j}^{(p)} d x\right)=-\bar{l}_{j}(u)+\sum_{i=1}^{2 m} r_{i} k_{j i}
$$

ya que $l_{i}\left(N_{j}\right)=\delta_{i j}$, siendo $k_{i j}=k_{j i}=\sum_{p=0}^{m} \int_{a}^{b} a_{p} N_{i}^{(p)} N_{j}^{(p)} d x$, de donde se deduce la simetria de

$\widetilde{K}$. Obsérvese que esta simetria puede obtenerse directamente de la reciprocidad dada en (2.12) para $u=N_{i}, v=N_{j}$.

Por otra parte de (2.8), (2.9) y (2.10) para $u$ arbitraria y $v$ tal que $L v=0$ se tiene

de donde se deduce que

$$
a(u, v)=\sum_{p=1}^{2 m} l_{p}(u) \bar{l}_{p}(v)
$$

$$
a\left(N_{i}, N_{j}\right)=k_{i j}=k_{j i}=\bar{l}_{i}\left(N_{j}\right)
$$

expresión que tiene gran interés ya que permite calcular también la matriz $\widetilde{K}$ mediante derivación.

De la relación (4.3) para cada $j=1, . ., 2 m$, resulta, en consecuencia, la relación matricial (4.1). Obsérvese que dicha relación matricial se puede poner equivalentemente en la forma particionada siguiente que expresamos en términos de $u$ directamente (con la términologia correspondiente a $(a, b)$ que es más precisa, especialmente para el ensamblado posterior de los términos $\widetilde{q}\left(a^{+}\right)$y $\widetilde{q}\left(b^{-}\right)$)

$$
\left[\begin{array}{ll}
K_{11}^{(a, b)} & K_{12}^{(a, b)} \\
K_{21}^{(a, b)} & K_{22}^{(a, b)}
\end{array}\right]\left[\begin{array}{l}
\tilde{u}\left(a^{+}\right) \\
\tilde{u}\left(b^{-}\right)
\end{array}\right]=\left[\begin{array}{l}
\widetilde{q}\left(a^{+}\right) \\
\widetilde{q}\left(b^{-}\right)
\end{array}\right]
$$

donde las matrices $K_{i j}^{(a, b)}, i, j=1,2$ son de dimensión $m x m, \tilde{u}\left(a^{+}\right)=\left(u(a), . ., u^{(m-1)}(a)\right)^{t}$,

$$
\tilde{u}\left(b^{-}\right)=\left(u(b), . ., u^{(m-1)}(b)\right)^{t}, \widetilde{q}\left(a^{+}\right)=\left(\bar{l}_{1}(u), . ., \bar{l}_{m}(u)\right)^{t}, \widetilde{q}\left(b^{-}\right)=\left(\bar{l}_{m+1}(u), . ., \bar{l}_{2 m}(u)\right)^{t} .
$$

Es interesante señalar el paralelismo del desarrollo realizado, con los elementos finitos o el análisis matricial de estructuras cuando se aplica el método de los desplazamientos. En este sentido, la construcción realizada aquí no es más que una extensión de los resultados, en dichas áreas, correspondientes a los problemas de segundo y cuarto orden relativos a los operadores diferenciales indicados en (3.3). Podemos, por tal motivo, reivindicar para este campo de los splines la terminologia 
estructural y denominar a la matriz $\widetilde{K}$ como matriz de rigidez del elemento spline, a $\tilde{r}$ o $\tilde{u}$ vector de desplazamientos nodales del elemento y a $\widetilde{q}$ vector de cargas nodales de equilibrio o de contorno del elemento. Desde un punto de vista mecánico cada componente del vector de desplazamientos puede considerarse que es dual o conjugada en el sentido de una cierta energia o trabajo, de la correspondiente componente del vector de acciones nodales de equilibrio. Nótese que, a diferencia con el método de elementos finitos, aqui no se ha realizado ninguna aproximación sobre la función $u$ en $[a, b]$, al emplear directamente una base de funciones formada por elementos que verifican la ecuación diferencial homogénea $L(u)=0$. Tomando ahora $v=\sum_{i=1}^{2 m} s_{i} N_{i}$, con $s_{i}$ datos arbitrarios, puede comprobarse de manera inmediata a partir de (2.10) y de $L v=0$ que la forma bilineal simétrica $a(u, v)$ se puede poner como

$$
a(u, v)=\int_{a}^{b}\left(\sum_{j=0}^{m} a_{j} D^{j} u D^{j} v\right) d x=\sum_{i=1}^{2 m} l_{i}(u) \bar{l}_{i}(v)=\widetilde{s}^{t} \widetilde{K} \widetilde{r}
$$

de donde resulta

$$
\int_{a}^{b}(L u) v d x=\widetilde{s}^{t}(\widetilde{K} \tilde{r}-\widetilde{q})
$$

o equivalentemente, utilizando la expresión matricial particionada en términos directamente de $u$ y $v$

$$
\int_{a}^{b}(L u) v d x=\left(\widetilde{v}\left(a^{+}\right)^{t}, \widetilde{v}\left(b^{-}\right)^{t}\right)\left(\left[\begin{array}{ll}
K_{11}^{(a, b)} & K_{12}^{(a, b)} \\
K_{21}^{(a, b)} & K_{22}^{(a, b)}
\end{array}\right]\left[\begin{array}{c}
\tilde{u}\left(a^{+}\right) \\
\widetilde{u}\left(b^{-}\right)
\end{array}\right]-\left[\begin{array}{c}
\widetilde{q}\left(a^{+}\right) \\
\widetilde{q}\left(b^{-}\right)
\end{array}\right]\right)
$$

Finalmente, el resultado de reciprocidad indicado en (2.12) se puede expresar, con la notación de este apartado, en la forma

$$
\tilde{u}^{t} \widetilde{q}_{v}=\widetilde{v}^{t} \widetilde{q}_{u}
$$

donde $\widetilde{q}_{u}$ y $\widetilde{q}_{v}$ son las acciones nodales que respectivamente corresponden a dos funciones $u, v$ definidas en $[a, b]$ y pertenecientes al núcleo del operador $L$. Este resultado se interpreta después en el apartado relativo a la ecuación de equilibrio global.

\subsection{Ejemplos de construcción de la ecuación de equilibrio local}

Exponemos a continuación, con propósitos ilustrativos, tres ejemplos de construcción de la ecuación de equilibrio local. En primer lugar abordamos el caso de los splines de quinto grado, en segundo lugar el de los splines en tensión para la situación particular de coeficientes constantes en cada subintervalo y finalmente,como tercer caso, el análogo al de la viga sobre fundación elástica. Dicho caso, en términos de splines, podria denominarse como el de splines tipo Winkler.

El operador diferencial para el primer caso es

$$
L(u)=(-1)^{3} D^{3}\left(a_{3}(x) D^{3} u\right)=-A u^{(6)}
$$

donde la función $a_{3}(x)=A>0$ es constante en cada subintervalo $(a, b)$. Dicha constante puede variar de unos intervalos a otros, teniéndose, en dicho caso, los correspondientes splines de quinto grado con peso. Los operadores $\bar{l}_{i}, i=1, . ., 6$ se obtienen aplicando $(2.7)$ y $(2.11)$

$$
\begin{array}{ll}
l_{1}(u)=-P_{0}(u)_{x=a}=-A u{ }^{(5)}\left(a^{+}\right), & \bar{l}_{2}(u)=-P_{1}(u)_{x=a}=A u^{(4)}\left(a^{+}\right), \\
i_{3}(u)=-P_{2}(u)_{x=a}=-A u{ }^{(3)}\left(a^{+}\right), & \bar{l}_{4}(u)=P_{0}(u)_{x=b}=A u{ }^{(5)}\left(b^{-}\right), \\
i_{5}(u)=P_{1}(u)_{x=b}=-A u{ }^{(4)}\left(b^{-}\right), & i_{6}(u)=P_{2}(u)_{x=b}=A u(3)\left(b^{-}\right)
\end{array}
$$

de esta manera queda definido el vector $\widetilde{q}$ o equivalentemente los vectores $\widetilde{q}\left(a^{+}\right)$y $\widetilde{q}\left(b^{-}\right)$. La matriz de rigidez del elemento spline se calcula mediante la primera expresión de (4.2). Teniendo en cuenta que la base de funciones de forma locales $\left\{N_{i}, i=1, . ., 6\right\}$ está formada por los siguientes polinomios

$$
\begin{array}{ll}
N_{1}=\left(l^{5}-10 l^{2} z^{3}+15 l z^{4}-6 z^{5}\right) / l^{5}, & N_{2}=z\left(l^{4}-6 l^{2} z^{2}+8 l z^{3}-3 z^{4}\right) / l^{4}- \\
N_{3}=z^{2}\left(l^{3}-3 l^{2} z+3 l z^{2}-z^{3}\right) /\left(2 l^{3}\right), & N_{4}=z^{3}\left(10 l^{2}-15 l z+6 z^{2}\right) / l^{5} \\
N_{5}=z^{3}\left(-4 l^{2}+7 l z-3 z^{2}\right) / l^{4}, & N_{6}=z^{3}\left(l^{2}-2 l z+z^{2}\right) /\left(2 l^{3}\right)
\end{array}
$$


donde $z=x-a$ y $l=b-a$ es la longitud del subintervalo, aplicando $k_{i j}=k_{j i}=\bar{l}_{i}\left(N_{j}\right)$ resulta

$$
\widetilde{K}=A\left[\begin{array}{cccccc}
720 / l^{5} & 360 / l^{4} & 60 / l^{3} & -720 / l^{5} & 360 / l^{4} & -60 / l^{3} \\
360 / l^{4} & 192 / l^{3} & 36 / l^{2} & -360 / l^{4} & 168 / l^{3} & -24 / l^{2} \\
60 / l^{3} & 36 / l^{2} & 9 / l & -60 / l^{3} & 24 / l^{2} & -3 / l \\
-720 / l^{5} & -360 / l^{4} & -60 / l^{3} & 720 / l^{5} & -360 / l^{4} & 60 / l^{3} \\
360 / l^{4} & 168 / l^{3} & 24 / l^{2} & -360 / l^{4} & 192 / l^{3} & -36 / l^{2} \\
-60 / l^{3} & -24 / l^{2} & -3 / l & 60 / l^{3} & -36 / l^{2} & 9 / l
\end{array}\right]
$$

El operador diferencial para el caso de los splines en tensión es

$$
L(u)=(-1)^{2} D^{2}\left(a_{2}(x) D^{2} u\right)+(-1) D\left(a_{1}(x) D u\right)=A u^{(4)}-B u^{(2)}
$$

donde las funciones $a_{3}(x), a_{1}(x)$ en el subintervalo genérico $(a, b)$ son constantes dadas respectivamente por $A>0$ y $B>0$. Estas constantes de igual manera que en el caso anterior pueden variar de unos subintervalos a otros, teniéndose los splines en tensión con peso. Precisamente esta posibilidad de modificar los valores de las constantes citadas, especialmente el de la $B$ que define la tensión, o mejor la tracción, es lo que ha dado gran popularidad a esta clase de splines en el campo de los gráficos. Los operadores que nos definen las cargas nodales de equilibrio

$$
\widetilde{q}\left(a^{+}\right)=\left(\bar{l}_{1}(u), \bar{l}_{2}(u)\right)^{t}, \widetilde{q}\left(b^{-}\right)=\left(\bar{l}_{3}(u), \bar{l}_{4}(u)\right)^{t}
$$

son

$$
\begin{array}{ll}
\bar{l}_{1}(u)=-P_{0}(u)_{x=a}=A u^{(3)}\left(a^{+}\right)-B u^{\prime}\left(a^{+}\right), & \bar{l}_{2}(u)=-P_{1}(u)_{x=a}=-A u^{(2)}\left(a^{+}\right), \\
\bar{l}_{3}(u)=-P_{0}(u)_{x=b}=-A u^{(3)}\left(b^{-}\right)+B u^{\prime}\left(b^{-}\right), & \bar{l}_{4}(u)=P_{1}(u)_{x=b}=A u^{(2)}\left(b^{-}\right),
\end{array}
$$

Teniendo en cuenta que las raíces de la ecuación característica correspondiente a la ecuación homogénea $L(u)=0$ son, 0 , doble y, $\pm r$, con $r=\sqrt{B / A}$, la base de funciones de forma es, tal y como puede calcularse de manera inmediata $\tilde{N}=\left(N_{1}, N_{2}, N_{3}, N_{4}\right)^{t}=C \tilde{x}$ donde

$$
\tilde{x}^{t}=(1, z, \operatorname{senh}(r z), \cosh (r z)), z=x-a
$$

con

$$
\begin{gathered}
C=\frac{1}{c_{4}}\left[\begin{array}{cccc}
c_{3} c_{1}-c_{2}+1 & -r c_{1} & c_{1} & 1-c_{2} \\
\left(c_{3} c_{2}-c_{1}\right) / r & 1-c_{2} & \left(c_{3} c_{2}-c_{2}+1\right) / r & c_{1}-c_{3} c_{2} \\
1-c_{2} & r c_{1} & -c_{1} & c_{2}-1 \\
\left(c_{1}-c_{3}\right) / r & 1-c_{2} & \left(c_{2}-1\right) / r & \left(c_{3}-c_{1}\right) / r
\end{array}\right] \\
l=b-a, c_{1}=\operatorname{senh}(r l), c_{2}=\cosh (r l), c_{3}=r l, c_{4}=2+c_{3} c_{1}-2 c_{2}
\end{gathered}
$$

Aplicando ahora $k_{i j}=k_{j i}=\bar{l}_{i}\left(N_{j}\right)$ resulta la matriz de rigidez local de los splines en tensión

$$
\tilde{K}=\frac{A}{c_{4}}\left[\begin{array}{cccc}
r^{3} c_{1} & r^{2}\left(c_{2}-1\right) & -r^{3} c_{1} & r^{2}\left(c_{2}-1\right) \\
r^{2}\left(c_{2}-1\right) & r\left(c_{3} c_{2}-c_{1}\right) & r^{2}\left(1-c_{2}\right) & r\left(c_{1}-c_{3}\right) \\
-r^{3} c_{1} & r^{2}\left(1-c_{2}\right) & r^{3} c_{1} & r^{2}\left(1-c_{2}\right) \\
r^{2}\left(c_{2}-1\right) & r\left(c_{1}-c_{3}\right) & r^{2}\left(1-c_{2}\right) & r\left(c_{3} c_{2}-c_{1}\right)
\end{array}\right]
$$

Finalmente el operador diferencial para el último caso es

$$
L(u)=(-1)^{2} D^{2}\left(a_{2}(x) D^{2} u\right)+a_{0}(x) u=A u^{(4)}+k u
$$

donde las funciones $a_{3}(x), a_{0}(x)$ en el subintervalo genérico $(a, b)$ son constantes dadas respectivamente por $A>0$ y $k>0$. Estas constantes como se ha indicado en los ejemples anteriores pueden variar de unos intervalos a otros, teniéndose los correspondientes casos con peso. Los operadores que definen en este ejemplo las cargas nodales de equilibrio 
son

$$
\widetilde{q}\left(a^{+}\right)=\left(\bar{l}_{1}(u), \bar{l}_{2}(u)\right)^{t}, \widetilde{q}\left(b^{-}\right)=\left(\bar{l}_{3}(u), \bar{l}_{4}(u)\right)^{t}
$$

$$
\begin{aligned}
& \bar{l}_{1}(u)=-P_{0}(u)_{x=a}=A u^{(3)}\left(a^{+}\right), \quad \bar{l}_{2}(u)=-P_{1}(u)_{x=a}=-A u^{(2)}\left(a^{+}\right), \\
& \bar{l}_{3}(u)=-P_{0}(u)_{x=b}=-A u^{(3)}\left(b^{-}\right), \quad \bar{l}_{4}(u)=P_{1}(u)_{x=b}=A u^{(2)}\left(b^{-}\right),
\end{aligned}
$$

Puede observarse que las formas lineales $\bar{l}_{i}, i=1, . ., 4$ son idénticas a las de los splines cúbicos con peso, aunque las funciones de forma en lugar de ser polinomios cúbicos, son, como en el caso anterior, funciones trascendentes. Las raices de la ecuación característica son los cuatro números complejos siguientes $( \pm r \pm i r)$, con $r=\sqrt[4]{C /(4 A)}$. Los elementos de la base los expresamos del mismo modo que en el caso anterior como

$$
\tilde{N}=\left(N_{1}, N_{2}, N_{3}, N_{4}\right)^{t}=C \tilde{x}
$$

donde

$$
\begin{aligned}
& \tilde{x}^{t}=(\cos (r z) \cosh (r z), \cos (r z) \operatorname{senh}(r z), \operatorname{sen}(r z) \cosh (r z), \operatorname{sen}(r z) \operatorname{senh}(r z)) \\
& z=x-a
\end{aligned}
$$

con

$$
\begin{aligned}
& C=\frac{1}{r c_{5}}\left[\begin{array}{cccc}
1 & -r\left(c_{1} c_{2}+c_{3} c_{4}\right) & r\left(c_{1} c_{2}+c_{3} c_{4}\right) & r\left(c_{4}^{2}-c_{2}^{2}\right) \\
0 & c_{4}^{2}-1 & c_{2}^{2}-1 & c_{3} c_{4}-c_{1} c_{2} \\
0 & r\left(c_{1} c_{4}+c_{2} c_{3}\right) & -r\left(c_{1} c_{4}+c_{2} c_{3}\right) & 2 r c_{1} c_{3} \\
0 & -c_{1} c_{3} & c_{1} c_{3} & c_{1} c_{4}-c_{2} c_{3}
\end{array}\right] \\
& c_{1}=\operatorname{senh}(r l), c_{2}=\cosh (r l), c_{3}=\operatorname{sen}(r l), c_{4}=\cos (r l), c_{5}=c_{2}^{2}+c_{4}^{2}-2
\end{aligned}
$$

Finalmente la matriz de rigidez, que se calcula en la forma ya indicada, es

$$
\widetilde{K}=\frac{A}{c_{5}}\left[\begin{array}{cccc}
4 r^{3}\left(c_{1} c_{2}+c_{3} c_{4}\right) & 2 r^{2}\left(c_{2}^{2}-c_{4}^{2}\right) & -4 r^{3}\left(c_{1} c_{4}+c_{2} c_{3}\right) & 4 r^{2} c_{1} c_{3} \\
2 r^{2}\left(c_{2}^{2}-c_{4}^{2}\right) & 2 r\left(c_{1} c_{2}-c_{3} c_{4}\right) & -4 r^{2} c_{1} c_{3} & 2 r\left(c_{2} c_{3}-c_{1} c_{4}\right) \\
-4 r^{3}\left(c_{1} c_{4}+c_{2} c_{3}\right) & -4 r^{2} c_{1} c_{3} & 4 r^{3}\left(c_{1} c_{2}+c_{3} c_{4}\right) & 2 r^{2}\left(c_{4}^{2}-c_{2}^{2}\right) \\
4 r^{2} c_{1} c_{3} \cdot & 2 r\left(c_{2} c_{3}-c_{1} c_{4}\right) & 2 r^{2}\left(c_{4}^{2}-c_{2}^{2}\right) & 2 r\left(c_{1} c_{2}-c_{3} c_{4}\right)
\end{array}\right]
$$

NOTA: Los dos últimos ejemplos expuestos son casos particulares del correspondiente al operador definido al final de (3.3). Cuando los coeficientes son constantes en cada subintervalo $(a, b)$, es decir, $A(x)=A, B(x)=B$ y $C(x)=k$ la ecuación característica $A r^{4}-B r^{2}+k=0$ es bicuadrada y por tanto se podría dar también en forma simbólica como en los casos anteriores $y$, de una manera más o menos directa, en función de los coeficientes, las expresiones de las funciones de forma y de la matriz de rigidez. No obstante dado el elevado número de casos posibles y, por otra parte, las facilidades actuales de utilización de herramientas de cálculo simbólico, puede ser más interesante, a efectos de cálculo, seguir para cada caso no trivial $(A>0, B \neq 0, k>0)$ el proceso indicado en la teoria, es decir, calcular la matriz de rigidez $\widetilde{K}$ del elemento spline mediante $k_{i j}=k_{j i}=\bar{l}_{i}\left(N_{j}\right)$ donde las formas lineales $\bar{l}_{i}$ son aqui las mismas que se indican en (4.7) con la particularidad de que el valor de $B$ (tracción o compresión) puede ser positivo o negativo. Asimismo, las funciones de interpolación $N_{i}, i=1, . ., 4$ se determinan en la forma usual, esto es, mediante $\widetilde{N}=C \widetilde{x}$ donde $\tilde{x}^{t}=\left(\varphi_{1}, \varphi_{2}, \varphi_{3}, \varphi_{4}\right)$ está constituido por un conjunto de funciones que forman un sistema fundamental para

$$
L(u)=A u^{(4)}-B u^{(2)}+k u=0
$$

y la matriz $C$ de cambio de base es la inversa y traspuesta de la matriz $M$, es decir $C=M^{-t}$ donde los elementos de $M$ son $m_{i j}=l_{i}\left(\varphi_{j}\right), i, j=1, . ., 4$, tal y como se deduce al imponer $l_{i}\left(N_{j}\right) \equiv \delta_{i j}$ : 


\subsection{Ecuación de equilibrio global del spline generalizado}

A continuación se obtiene la expresión matricial de la ecuación de equilibrio global del spline generalizado. Basta emplear las expresiones (4.4) y (4.5) para determinar la correspondiente expresión en $\left[x_{1}, x_{n}\right]$, teniendo en cuenta para ello las condiciones (3.1) y la suma de las contribuciones de cada subintervalo $\left(x_{i}, x_{i+1}\right), i=1, . ., n-1$, es decir

$$
\int_{x_{1}}^{x_{n}}(L u) v d x=\sum_{i=1}^{n-1}\left(\tilde{v}\left(x_{i}^{+}\right)^{t}, \tilde{v}\left(x_{i+1}^{-}\right)^{t}\right)\left(\left[\begin{array}{cc}
K_{11}^{i} & K_{12}^{i} \\
K_{21}^{i} & K_{22}^{i}
\end{array}\right]\left[\begin{array}{c}
\tilde{u}\left(x_{i}^{+}\right) \\
\tilde{u}\left(x_{i+1}^{-}\right)
\end{array}\right]-\left[\begin{array}{c}
\tilde{q}\left(x_{i}^{+}\right) \\
\widetilde{q}\left(x_{i+1}^{-}\right)
\end{array}\right]\right)
$$

Como $L u=0$ en cada subintervalo y considerando que las funciones $u$ y $v$ son en el intervalo total de la clase $C^{m-1}$, es decir $\tilde{u}\left(x_{i}^{+}\right)=\tilde{u}\left(x_{i}^{-}\right)$en los nodos internos y lo mismo para $v$, se puede poner

$$
\begin{aligned}
& \mathbf{v}^{t}=\left(\tilde{v}\left(x_{1}\right)^{t}, . ., \tilde{v}\left(x_{n}\right)^{t}\right) \\
& \mathbf{u}^{t}=\left(\tilde{u}\left(x_{1}\right)^{t}, . ., \tilde{u}\left(x_{n}\right)^{t}\right)
\end{aligned}
$$

resulta, empleando las expresiones expandidas usuales de los elementos finitos para la matriz de rigidez y vector de acciones nodales de equilibrio de cada elemento, la expresión

$$
\int_{x_{1}}^{x_{n}}(L u) v d x=\mathbf{v}^{\mathbf{t}}(\mathbf{K} \mathbf{u}-\mathbf{Q})=\mathbf{0}
$$

donde $\mathbf{K}$ y $\mathbf{Q}$ equivalen al ensamblado habitual de las correspondientes matrices locales y que aquí se obtienen comó suma de matrices expandidas que resultan, al ampliar con ceros, las matrices locales en la posición correspondiente hasta llegar a la dimensión $m \times n$, es decir

$$
\mathbf{K}=\sum_{i=1}^{n-1}\left[\begin{array}{cccc}
\ddots & \vdots & \vdots & \cdot \\
\cdots & K_{11}^{i} & K_{21}^{i} & \cdots \\
\cdots & K_{21}^{i} & K_{22}^{i} & \cdots \\
\vdots & \vdots & \vdots & \ddots
\end{array}\right]_{(m \times n) \times(m \times n)} \quad \mathbf{Q}=\sum_{i=1}^{n-1}\left[\begin{array}{c}
\vdots \\
\widetilde{q}\left(x_{i}^{+}\right) \\
\widetilde{q}\left(x_{i+1}^{-}\right) \\
\vdots
\end{array}\right]_{(m \times n) \times 1}
$$

Por otra parte, como la relación (4.8) se verifica para cualquier $\mathbf{v}$, se tiene la siguiente expresión que, por la analogia ya citada, podemos denominar ecuación de equilibrio global del spline generalizado

$$
\mathbf{K u}=\mathbf{Q}
$$

donde $\mathbf{K}$ es la matriz de rigidez global del spline generalizado, que es, simétrica y tridiagonal por bloques y $\mathbf{Q}$ es el vector de acciones nodales exteriores. Las columnas de la matriz de rigidez pueden interpretarse como las acciones nodales que resultan para los desplazamientos dados por las funciones de forma globales. Dichas funciones de forma globales se obtienen a partir de las locales en la forma usual y son splines generalizados cuyas componentes del vector de desplazamientos, son todas nulas excepto una, que toma el valor unidad.

La expresión matricial de la forma bilineal $a(u, v)$ para las funciones $u$ y $v$ pertenecientes al espacio de los splines generalizados es

$$
a(u, v)=\sum_{i=0}^{n-1} \int_{x_{i}}^{x_{i+1}}\left(\sum_{j=0}^{m} a_{j} D^{j} u D^{j} v\right) d x=\mathbf{v}^{\mathbf{t}} \mathbf{K u}
$$

Formalmente se podria definir la energía de deformación del spline generalizado como

$$
\frac{1}{2} a(u, u)=\frac{1}{2} \mathbf{u}^{\mathbf{t}} \mathbf{K u}=\frac{1}{2} \mathbf{u}^{\mathbf{t}} \mathbf{Q}
$$

para aquellos casos donde el operador $L$ verifique las condiciones de no negatividad

$$
a_{j}(x) \geq 0, j=1, . ., m-1, a_{m} \geq \delta>0, x \in\left[x_{1}, x_{n}\right]
$$

Se debe destacar que la expresión (4.9) es una identidad resultante de la fórmula de Green (2.2) aplicada al caso del operador autoadjunto definido en (2.5) y, por tanto, es satisfecha por cualquier spline generalizado definido en la forma (3.1) con las condiciones de regularidad dadas en (3.2). Es importante 
señalar que dicha expresión (4.9) únicamente pone de manifiesto que, fijados los desplazamientos de los nodos mediante el vector u se tienen, de manera univoca, las acciones en los nodos definidas por el vector $\mathbf{Q}$.

Si distinguimos ahora con un subindice el origen de las acciones y si $u$ y $v$ representan dos splines generalizados cualesquiera, relativos a los mismos nodos, de la simetría de la matriz de rigidez global y de las expresiones $\mathbf{K u}=\mathbf{Q}_{u}, \mathbf{K v}=\mathbf{Q}_{v}$ se tiene el siguiente resultado

$$
\mathbf{v}^{t} \mathbf{Q}_{u}=\mathbf{u}^{\mathrm{t}} \mathbf{Q}_{v}
$$

que es análogo al teorema de reciprocidad en mecánica de estructuras y que aqui se interpreta de la forma siguiente:

( V) Para dos splines generalizados cualesquiera relativos a los mismos nodos, la suma de los productos de los desplazamientos nodales del primero por las acciones nodales del segundo es igual a la suma de los productos de los desplazamientos nodales del segundo por las acciones nodales del primero.

También se tiene trivialmente $\mathbf{Q}_{u+v}=\mathbf{Q}_{u}+\mathbf{Q}_{v}$, que representa el principio de superposición de acciones nodales para un spline generalizado que es suma de otros dos.

\subsection{Problemas de interpolación con splines generalizados. Propiedades de mínimo}

Cabe ahora definir diferentes problemas de interpolación para este planteamiento. El enfoque usual en la teoria de splines generalizados ([1, pp. 195, 196]) consiste en imponer en cada uno de los nodos condiciones de interpolación de tipo Hermite, esto es, fijar los valores de las ordenadas y cierto número de derivadas sucesivas en cada uno de los nodos, en la forma

$$
u^{(j)}\left(x_{i}\right)=y_{i k}, i=1, . ., n ; j=0,1, . ., z-1
$$

donde el valor de $z$ es la deficiencia, tal que $1 \leq z \leq m$ (obsérvese que en el apartado tres de este trabajo permitimos que la deficiencia pueda ser diferente en los distintos nodos internos). Con estas condiciones el objetivo es calcular una función del espacio de splines generalizados que sea de la mayor regularidad posible,es decir, de la clase $C^{2 m-1-z}$ en $\left[x_{1}, x_{n}\right]$.

Nuestro planteamiento, sin embargo, permite resolver problemas de interpolación más generales, donde podemos imponer para cada nodo $x_{i}, i=1, . ., n$ y para cada orden de derivación, condiciones de interpolación del tipo Hermite-Birkhoff, definidas a partir de la matriz de incidencia ([52, p. 248], [5 p. 75]) $E=\left(E_{i j}\right), E_{i j} \in\{0,1\}, i=1, . ., n, j=1, . ., m$ en la forma siguiente: si $E_{i j}=1$ esto quiere decir que fijamos el dato $u^{(j-1)}\left(x_{i}\right)$, no fijándose en el caso de que $E_{i j}=0$. Es decir se dan algunas de las componentes del vector de desplazamientos

$$
\mathbf{u}^{t}=\left(u\left(x_{1}\right), . ., u^{(m-1)}\left(x_{1}\right), . ., u\left(x_{n}\right), . ., u^{(m-1)}\left(x_{n}\right)\right)
$$

de modo que en cada nodo las derivadas datos no requieren ser consecutivas y en diferentes nodos las derivadas fijadas pueden ser distintas, es decir, los datos que se fijan constituyen una sucesión, que para cada nodo (en los que se den datos únicamente), no es obligatoriamente completa ni correlativa. Por otro lado el planteamiento propuesto permite fijar además y simultáneamente, las componentes del vector de acciones nodales exteriores que son duales de las componentes no prefijadas del vector de desplazamientos. Dichas componentes son

$$
\begin{aligned}
& \mathbf{Q}^{\prime}=\left(-P_{0}(u)_{x=x_{1}^{+}}, . .,-P_{m-1}(u)_{x=x_{1}^{+}}, P_{0}(u)_{x=x_{2}^{-}}-P_{0}(u)_{x=x_{2}^{+}}, . ., P_{m-1}(u)_{x=x_{2}^{-}}-P_{m-1}(u)_{x=x_{2}^{+}}, . .\right. \\
& \left.. ., P_{0}(u)_{x=x_{n-1}^{-}}-P_{0}(u)_{x=x_{n-1}^{+}}, . ., P_{m-1}(u)_{x=x_{n-1}^{-}}-P_{m-1}(u)_{x=x_{n-1}^{+}}, P_{0}(u)_{x=x_{n}^{-}}, . ., P_{m-1}(u)_{x=x_{n}^{-}}\right)
\end{aligned}
$$

En resumen puede plantearse el siguiente problema de interpolación. Determinar el spline generalizado definido por las condiciones (3.1) y (3.2) tales que se conocen para el mismo $p$ componentes de las

$n \times m$ del vector $\mathbf{u}$ de desplazamientos nodales y $s=n \times m-p$ componentes del vec̄or $\mathbf{Q}$ de acciones nodales, ocupando, estas últimas, posiciones complementarias respecto a las componentes 
reordenar filas y columnas, el sistema de ecuaciones siguiente, donde $\mathbf{u}_{\mathbf{p}}^{*}, \mathbf{Q}_{\mathbf{s}}^{*}$ son los datos y $\mathbf{u}_{\mathbf{s}}, \mathbf{Q}_{\mathbf{p}}$ son las incógnitas a determinar

$$
\left[\begin{array}{ll}
K_{p p} & K_{p s} \\
K_{s p} & K_{s s}
\end{array}\right]\left[\begin{array}{l}
u_{p}^{*} \\
u_{s}
\end{array}\right]=\left[\begin{array}{l}
Q_{p} \\
Q_{s}^{*}
\end{array}\right]
$$

El problema tiene solución única si la submatriz $\mathbf{K}_{\mathbf{s s}}$ es regular, resultando formalmente en dicho caso

$$
\mathbf{u}_{\mathrm{s}}=\mathbf{K}_{\mathrm{ss}}^{-1}\left(\mathbf{Q}_{\mathrm{s}}^{*}-\mathbf{K}_{\mathrm{sp}} \mathbf{u}_{\mathrm{p}}^{*}\right), \mathbf{Q}_{\mathrm{p}}=\mathbf{K}_{\mathrm{pp}} \mathbf{u}_{\mathrm{p}}^{*}+K_{\mathrm{ps}} \mathbf{u}_{\mathrm{s}}
$$

Obsérvese que cuando el problema de interpolación está bien planteado, es decir, cuando tiene solución única, las acciones quedan unívocamente determinadas en aquellos puntos donde se han fijado los desplazamientos y, reciprocamente, los desplazamientos quedan unívocamente determinados en donde se han fijado las acciones. Véase que entre los problemas de interpolación posibles también se encuentran aquellos casos extremos donde o bien se fijan todos los desplazamientos o bien se fijan todas las acciones nodales. El primero es un problema de interpolación de Hermite a trozos en el espacio de splines generalizados donde, independientemente de la regularidad o no de la matriz de coeficientes de (4.9) el vector de acciones nodales queda univocamente determinado. Por otra parte, la otra situación extrema requiere, precisamente, que la matriz citada sea regular, para que queden univocamente determinados todos los desplazamientos.

Esta forma tan general de imponer las condiciones de interpolación, fijando algunas componentes del vector que hemos denominado de desplazamientos, coincide, en parte, con las que se emplean para tipos particulares de L-splines como son los splines heterogéneos ([1, p. 194,195]), Lg-splines ([26, p.106]), $\gamma$ splines ([53, p.12]). Sin embargo, en ninguno de estos tipos se trata la asignación de valores a las correspondientes componentes del vector $\mathbf{Q}$ ya que por la formulación alli efectuada, diferente a nuestra formulación matricial, el vector citado de acciones nodales exteriores, no queda determinado explicitamente. EI valor que consecuentemente por defecto queda asignado para las correspondientes componentes del vector $\mathbf{Q}$ es, precisamente, el valor nulo.

Respecto a la unicidad de solución del problema de interpolación con splines generalizados planteado, se tiene el siguiente resultado:

( VI ) Suponiendo que el operador diferencial $L$ verifica además de las condiciones (3.2) las condiciones de no negatividad $a_{j}(x) \geq 0, j=0, . ., m-1$ en $\left[x_{1}, x_{n}\right]$, y las longitudes de cada subintervalo son tales que se verifica ( III ), entonces el problema de interpolación de splines generalizados planteado tiene solución única $y$ equivalentemente $\mathbf{K}_{\mathrm{ss}}$ es regular, si la única solución del problema de Hermite-Birkhoff de obtener un polinomio $p_{k-1}(x)$ (de grado $k-1$ ) que verifica las mismas condiciones de interpolación que el spline pero con datos nulos, $u_{p}^{* *}=0$, es el polinomio nulo, siendo $k$ el mínimo de $\{0,1, . ., m\}$ tal que $a_{k}(x)>0$ en algún subconjunto de medida positiva del intervalo $\left[x_{1}, x_{n}\right]$.

En efecto, poniendo $\mathbf{u}_{\mathrm{p}}=\mathbf{u}_{\mathrm{p}}^{* *}=\mathbf{0}$ se deduce que $\mathbf{K}_{\mathrm{ss}}$ es semidefinida positiva ya que

$$
\begin{aligned}
& F\left(\mathbf{u}_{\mathbf{s}}\right)=\mathbf{u}_{\mathbf{s}}^{\mathbf{t}} \mathbf{K}_{\mathbf{s s}} \mathbf{u}_{\mathbf{s}}=\left[\begin{array}{ll}
\mathbf{0} & \mathbf{u}_{\mathbf{s}}^{\mathbf{t}}
\end{array}\right]\left[\begin{array}{ll}
\mathbf{K}_{\mathbf{p p}} & \mathbf{K}_{\mathbf{p s}} \\
\mathbf{K}_{\mathbf{s p}} & \mathbf{K}_{\mathbf{s s}}
\end{array}\right]\left[\begin{array}{c}
\mathbf{0} \\
\mathbf{u}_{\mathbf{s}}
\end{array}\right]= \\
& =\sum_{i=0}^{n-1} \int_{x_{i}}^{\alpha_{i+1}}\left(\sum_{j=0}^{m} a_{j}\left(D^{j} u\right)^{2}\right) d x=\int_{x_{1}}^{x_{n}}\left(\sum_{j=0}^{m} a_{j}\left(D^{j} u\right)^{2}\right) d x \geq 0
\end{aligned}
$$

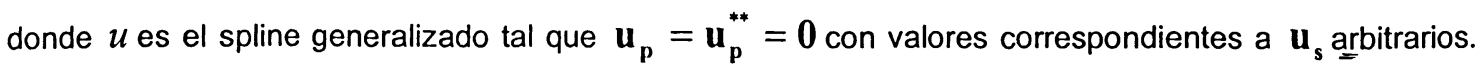
Para ver cuándo es definida positiva, ponemos $F\left(\mathbf{u}_{\mathbf{s}}\right)=0$ y determinamos el -valor de $k=\min \left\{0,1, . ., m\right.$ tal que $a_{k}(x)>0$ en algún subconjunto $S \subset\left[x_{1}, x_{n}\right]$ de medida de Lebesgue 
positiva (esta condición no es vacía ya que por hipótesis $a_{m} \geq \delta>0$ en todo $\left[x_{1}, x_{n}\right]$ ). En estas condiciones $u^{(m)}(x) \equiv 0$ en $\left[x_{1}, x_{n}\right]$, luego $u$ en cada subintervalo debe ser un polinomio de grado menor o igual que $m-1$, por otra parte si $k$ es estrictamente menor que $m$, de la condición $u^{(k)}(x) \equiv 0$ en $S$, se deduce que $u=p_{k-1}(x)$ es un polinomio de grado menor o igual que $k-1$ en $S$. Como por otra parte $u$ es de la clase $C^{m-1}$ en $\left[x_{1}, x_{n}\right]$ y de la clase $C^{2 m}$ en cada subintervalo $\left(x_{i}, x_{i+1}\right)$, el polinomio $u=p_{k-1}(x)$ queda prolongado a todo $\left[x_{1}, x_{n}\right]$. Si ahora el único polinomio de grado menor o igual a $k-1$ que verifica las condiciones dadas por $\mathbf{u}_{\mathbf{p}}=\mathbf{u}_{\mathbf{p}}^{* *}=0$ es el polinomio nulo, entonces $\mathbf{u}_{\mathbf{s}}=0 \mathrm{y}$ consecuentemente $\mathbf{K}_{\mathrm{ss}}$ es definida positiva y por tanto regular y el problema (4.12) tiene solución única.

La aplicación del resultado anterior, por ejemplo, al caso de interpolación con splines cúbicos con peso, indica que basta fijar al menos dos ordenadas o una ordenada y una pendiente en el conjunto de nodos $\left\{x_{1}, x_{2}, \ldots, x_{n}\right\}$, para que el problema tenga solución única. Esto es lo que, en el lenguaje de las estructuras, se expresa diciendo que las condiciones de vínculo de la viga deben ser tales que las condiciones de desplazamiento de valor nulo derivadas de ellas hagan que el único polinomio de primer grado que las verifique sea el polinomio nulo. Esto, como es bien conocido, se consigue físicamente con al menos dos apoyos o un apoyo y un empotramiento (no necesariamente en el mismo nodo).

Por otra parte, la aplicación al caso de los splines de quinto grado con peso, donde las condiciones en desplazamiento que se pueden fijar en cada nodo son ordenada, pendiente y derivada segunda, nos indica que basta con fijar, en el conjunto de nodos, al menos tres ordenadas, o una ordenada y dos pendientes, o dos ordenadas y una pendiente en un nodo que no sea punto medio de los dos donde se ha fijado la ordenada, etc., para que el spline de quinto grado quede univocamente determinado. Como puede verse estas condiciones son, de tal modo, que el único polinomio de segundo grado que las verifica con datos nulos es el polinomio nulo.

A continuación, en relación con aspectos energéticos, indicamos que, de modo análogo a los problemas lineales de elasticidad, se verifica, para los splines generalizados, el principio de energía potencial total minima. Supongamos que las condiciones de interpolación dadas por las componentes $\mathbf{u}_{\mathbf{p}}^{*}$ del vector de desplazamientos son tales que el correspondiente problema de interpolación de splines generalizados tiene solución única. Donde la solución se calcula para las acciones dadas por los datos simultáneamente impuestos definidos por el vector $\mathbf{Q}_{\mathbf{s}}^{*}$. Sea $g(x), x \in\left[x_{1}, x_{n}\right]$ una función arbitraria que verifica los datos dados por $\mathbf{u}_{\mathbf{p}}^{*}$, se define la energía potencial total para dicha función como el valor del funcional $F(g)$ donde

$$
F(g)=\frac{1}{2} \int_{x_{1}}^{x_{n}}\left(\sum_{j=0}^{m} a_{j}\left(D^{j} g\right)^{2}\right) d x-\mathbf{g}_{\mathbf{s}}^{t} \mathbf{Q}_{\mathbf{s}}^{*}
$$

donde $\mathbf{g}_{\mathbf{s}}$ son los desplazamientos (conocidos) de la función $g(x)$ correspondientes a las posiciones complementarias de las componentes de $\mathbf{u}_{\mathbf{p}}^{*}$. El primer sumando de (4.14) puede denominarse, por analogía con la mecánica de estructuras, energía de deformación. En el campo de los splines es habitual emplear únicamente esta energía de deformación pues el segundo sumando no aparece en la expresión por las razones ya comentadas, donde, al no considerarse en otras formulaciónes el vector de acciones nodales, el valor que por defecto queda asignado es $\mathbf{Q}_{\mathbf{s}}^{*}=\mathbf{0}$. Asimismo dicha energía de deformación es expresada en los textos y trabajos más conocidos, en términos del operador diferencial en forma factorizada donde la interpretación estructural, como se verá,es diferente.

NOTA: La expresión que define la energía tiene sentido cuando $g(x)$ pertenece al espacio de Sobolev ([54, p. 45]) $W_{2}^{m}\left[x_{1}, x_{n}\right] \circ H^{m}\left[x_{1}, x_{n}\right]$ formado por el conjunto de funciones reales $h$ tales que $D^{m-1} h$ es absolutamente continua en el intervalo dado y $D^{m} h$ es de cuadrado integrable en sentido de Lebesgue, es decir $D^{m} h \in L_{2}$, teniéndose que $H^{m}\left[x_{1}, x_{n}\right] \subset C^{m-1}\left[x_{1}, x_{n}\right]$. Dicho espacio de 
Sobolev puede verse como el resultado de completar el espacio de funciones $C^{\infty}\left[x_{1}, x_{n}\right]$ con respecto a la norma

Se tiene el siguiente resultado para la energia

$$
\|h\|_{H^{m}}=\left\{\int_{x_{1}}^{x_{2}}\left(\sum_{j=0}^{m}\left(D^{j} h\right)^{2}\right) d x\right\}^{1 / 2}
$$

\section{( VII ) El funcional de energía definido por (4.14) toma el valor mínimo cuando $g$ es el spline generalizado $u$ (único por hipótesis) que satisface (4.12).}

En efecto, llamando $v=g-u$ se tiene que $v \in H^{m}\left[x_{1}, x_{n}\right] \mathbf{v}_{\mathbf{p}}=\mathbf{v}_{\mathbf{p}}^{* *}=\mathbf{0}$, es decir, verifica las mismas condiciones de interpolación que $g$ y $u$ pero con valores nulos para las componentes fijadas del vector de desplazamientos.

$$
F(g)=F(u+v)=F(u)+\frac{1}{2} \int_{\alpha_{1}}^{x_{n}}\left(\sum_{j=0}^{m} a_{j}\left(D^{j} v\right)^{2}\right) d x+\left(\int_{x_{1}}^{x_{n}}\left(\sum_{j=0}^{m} a_{j} D^{j} u D^{j} v\right) d x-\mathbf{v}_{\mathbf{s}}^{t} \mathbf{Q}_{\mathbf{s}}^{*}\right)
$$

El tercer sumando del segundo miembro es nulo como se deduce de aplicar (2.9) a cada subintervalo $\left(x_{i}, x_{i+1}\right)$, y observar que $L(u)=0$ en dichos subintervalos. Por otra parte, el segundo sumando, en virtud del resultado ( $V I$ ) y por la hipótesis de unicidad realizada, es nulo únicamente cuando $v(x) \equiv 0$ en $\left[x_{1}, x_{n}\right]$.

A partir del resultado anterior se deriva de manera inmediata, para el operador en la forma usual dada por (2.5), lo que en el campo de los splines y para el operador diferencial en la forma factorizada (2.14) y con $\mathbf{Q}_{\mathrm{s}}^{*}=\mathbf{0}$ se conoce como primera relación integral. Ésta, en nuestro planteamiento, se expresaria como

$$
\frac{1}{2} \int_{\alpha_{1}}^{x_{n}}\left(\sum_{j=0}^{m} a_{j}\left(D^{j} g\right)^{2}\right) d x=\frac{1}{2} \int_{\alpha_{1}}^{x_{n}}\left(\sum_{j=0}^{m} a_{j}\left(D^{j}(g-u)\right)^{2}\right) d x+\frac{1}{2} \int_{x_{1}}^{x_{n}}\left(\sum_{j=0}^{m} a_{j}\left(D^{j} u\right)^{2}\right) d x
$$

de donde se deduce la siguiente propiedad de minimo

$$
\int_{\alpha_{1}}^{\alpha_{n}}\left(\sum_{j=0}^{m} a_{j}\left(D^{j} g\right)^{2}\right) d x \geq \int_{\alpha_{1}}^{\alpha_{n}}\left(\sum_{j=0}^{m} a_{j}\left(D^{j} u\right)^{2}\right) d x
$$

que puede interpretarse como que el spline generalizado que interpola los datos dados por $\mathbf{u}_{\mathbf{p}}^{*}$, es la función para la que la energia de deformación toma el valor mínimo.

Asimismo, cuando $g \in H^{2 m}\left[x_{1}, x_{n}\right]$ verifica las mismas condiciones de interpolación que el spline generalizado, dadas por los vectores de datos $\mathbf{u}_{p}^{*} y \mathbf{Q}_{s}^{*}$, entonces se tiene la relación que para el. operador en la forma factorizada se conoce como segunda relación integral

$$
\int_{x_{1}}^{x_{n}}(g-u) L(g)=\int_{\alpha_{1}}^{x_{n}}\left(\sum_{j=0}^{m} a_{j}\left(D^{j}(g-u)\right)^{2}\right) d x
$$

En efecto, basta aplicar para cada subintervalo la expresión (2.6) tomando las dos funciones iguales a $g-u$ y tener en cuenta que $L(g-u)=L(g)$ y que las componentes conocidas y calculadas por las expresiones (4.12) y (4.13) son (por hipótesis) las mismas para $g$ y $u$. De esta forma la expresión siguiente es nula

$$
\sum_{i=1}^{n-1}(-1) \sum_{k=0}^{m-1}\left((g-u)^{(k)} P_{k}(g-u)\right) \mid \begin{aligned}
& x_{i+1}^{-} \\
& x_{i}^{+}
\end{aligned}
$$




\subsection{Resultados para el caso donde el operador está en forma factorizada}

Indicamos a continuación y de manera muy esquemática la construcción de las ecuaciones de equilibrio local y global para los splines generalizados cuando el operador está en la forma factorizada dada por (2.14). Dando los mismos pasos que en el caso correspondiente al operador en la forma autoadjunta usual y considerando las expresiones (2.17), (2.18) y (2.4) y las mismas hipótesis respecto a la existencia de las funciones de forma que constituyen la base de Lagrange para la interpolación de Hermite generalizada relativa al intervalo $[a, b]$, se tiene el siguiente resultado análogo a (IV)

( VIII ) Sea $L(u)=L_{1}^{*}\left(L_{1}(u)\right)$ el operador diferencial autoadjunto en forma factorizada, $l_{k}, k=1, . ., 2 m$ y $\bar{l}_{k}, k=1, . ., 2 m$ las formas lineales definidas en (2.4) y la forma bilineal simétrica $a_{1}(u, v)$ correspondiente. La solución de $L u=0$ en $[a, b]$ tal que

$$
l_{k}(u)=u^{(k-1)}(a)=r_{k}, l_{k+m}(u)=u^{(k-1)}(b)=r_{k+m}, k=1, . ., m
$$

donde $r_{k}, k=1, . ., 2 m$ son números reales arbitrarios dados, verifica la siguiente relación matricial que denominamos ecuación de equilibrio local o ecuación de equilibrio del elemento de spline generalizado en el intervalo $[a, b]$

$$
\widetilde{K}_{1} \tilde{u}=\widetilde{q}_{1}
$$

donde las matrices $\widetilde{K}_{1}=\left(k_{i j}\right)_{i, j=1, . ., 2 m}, \tilde{u}$ y $\widetilde{q}_{1}$ son en este caso

$$
\begin{aligned}
k_{i j} & =k_{j i}=\bar{l}_{i}\left(L_{1}\left(N_{j}\right)\right)=a_{1}(u, v)=\sum_{p=0}^{m} \int_{a}^{b} L_{1}\left(N_{i}\right) L_{1}\left(N_{j}\right) d x, i, j=1, . ., 2 m \\
\tilde{u}^{t} & =\tilde{r}^{t}=\left(r_{1}, . ., r_{m}, r_{m+1}, . ., r_{2 m}\right) \\
\tilde{q}_{1}^{t} & =\left(\bar{l}_{1}(u), . ., \bar{l}_{m}(u), \bar{l}_{m+1}(u), . ., \bar{l}_{2 m}(u)\right)= \\
& =\left(-p_{0}\left(L_{1}(u)\right)_{x=a}, . .,-p_{m-1}\left(L_{1}(u)\right)_{x=a}, p_{0}\left(L_{1}(u)\right)_{x=b}, . ., p_{m-1}\left(L_{1}(u)\right)_{x=b}\right)
\end{aligned}
$$

y las denominamos respectivamente como matriz de rigidez local, vector de desplazamientos nodales y vector de cargas nodales de equilibrio para el elemento de spline generalizado.

Asimismo, mediante el proceso usual de ensamblado se llega a la ecuación de equilibrio global $\mathbf{K}_{1} \mathbf{u}=\mathbf{Q}_{1}$ análoga a la (4.9) donde el vector de desplazamientos $\mathbf{u}$ es el mismo que el allí definido, sin embargo el vector de acciones nodales exteriores $\mathbf{Q}_{1}$ es diferente al definido en (4.11) al venir dadas la componentes de dicho vector en términos de la composición de los operadores $p_{i}, i=0, . ., m-1$ y $L_{1}$, distintos en su naturaleza a los $P_{i}, i=0, . ., m-1$ definidos en (2.7). Por la misma razón también es diferente la matriz $\mathbf{K}_{1}$. Asimismo la expresión de la forma bilineal análoga a la (4.10) es,en este caso,

$$
a_{1}(u, v)=\sum_{i=1}^{n-1} \int_{x_{i}}^{x_{i+1}} L_{1}(u) L_{1}(v) d x=v^{i} K_{1} \mathbf{u}
$$

Por otra parte, los resultados sobre la unicidad de solución del problema de interpolación análogo al planteado en (4.12) y asimismo las propiedades de minima energia potencial total y la primera y segunda relación integral se derivan de la misma forma, aunque las expresiones que se obtienen son diferentes. En la referencia [1] se realiza un estudio muy detallado de los splines generalizados utilizando la forma factorizada del operador, obteniendo la primera y segunda relación integral, entre otras propiedades; no obstante no aparece el concepto de acción nodal, ni en ningún punto se realizan desarrollos que puedan considerarse próximos al esquema matricial aqui propuesto.

Exponemos, con un ejemplo sencillo, el distinto significado de la matriz de rigidez y del vector de cargas nodales, dependiendo de que se emplee para el operador la forma autoadjunta usual o la factorizada.

Sea el operador correspondiente a la barra sometida a esfuerzos axiles y embebida en un mediō elástico

$$
L(u)=-\left(A(x) u^{\prime}\right)^{\prime}+k(x) u, x \in(a, b) .
$$


Consideremos el caso particular $A(x)=A($ cte $), k(x)=k($ cte $), x \in(a, b)$ donde un sistema fundamental de soluciones de $L(u)=0$ es $\left\{\varphi_{1}(x)=\exp \left(r_{1} x\right), \varphi_{2}(x)=\exp \left(r_{2} x\right)\right\}$ donde $r_{1}, r_{2}= \pm \sqrt{k / A}$. A partir de dicho sistema se obtiene, en la forma usual, la correspondiente base de Lagrange de funciones de forma $\left\{N_{1}, N_{2}\right.$. Para este caso $l_{1}(u)=u(a), l_{2}(u)=u(b)$ y las acciones nodales de equilibrio son $\bar{l}_{1}(u)=-P_{0}(u)_{x=a}=-A u^{\prime}(a), \bar{l}_{2}(u)=P_{0}(u)_{x=b}=A u^{\prime}(b)$. Los elementos de la matriz de rigidez local $\widetilde{K}$ se obtienen de $k_{i j}=k_{j i}=\bar{l}_{i}\left(N_{j}\right)$ resultando la ecuación de equilibrio local

$$
\left[\begin{array}{cc}
-A N_{1}^{\prime}(a) & -A N_{2}^{\prime}(a) \\
A N_{1}^{\prime}(b) & A N_{2}^{\prime}(b)
\end{array}\right]\left[\begin{array}{l}
u(a) \\
u(b)
\end{array}\right]=\left[\begin{array}{c}
-A u^{\prime}(a) \\
A u^{\prime}(b)
\end{array}\right]
$$

Si se emplea, por otra parte, la forma factorizada para el operador $L$, resulta $L(u)=L_{1}^{*}\left(L_{1}(u)\right)$ con $L_{1}(u)=\sqrt{A} u^{\prime}+\sqrt{k} u$ y $L_{1}^{*}(u)=-\sqrt{A} u^{\prime}+\sqrt{k} u$, siendo, para este caso, las acciones nodales

$$
\begin{aligned}
& \bar{l}_{1}\left(L_{1}(u)\right)=-p_{0}\left(L_{1}(u)\right)_{x=a}=-\sqrt{A}\left(\sqrt{A} u^{\prime}+\sqrt{k} u\right)_{x=a}=-A u^{\prime}(a)-\sqrt{A k} u(a) \\
& \bar{l}_{2}\left(L_{1}(u)\right)=p_{0}\left(L_{1}(u)\right)_{x=b}=\sqrt{A}\left(\sqrt{A} u^{\prime}+\sqrt{k} u\right)_{x=b}=A u^{\prime}(b)+\sqrt{A k} u(b)
\end{aligned}
$$

donde $p_{0}(\underline{u})=\sqrt{A} u$ tal y como se indica en (2.3). Los elementos de $\widetilde{K}_{1}$ se obtienen de la manera indicada en $V I I$, resultando la ecuación de equilibrio

$$
\left[\begin{array}{cc}
-A N_{1}^{\prime}(a)-\sqrt{A k} N_{1}(a) & -A N_{2}^{\prime}(a)-\sqrt{A k} N_{2}(a) \\
A N_{1}^{\prime}(b)+\sqrt{A k} N_{1}(b) & A N_{2}^{\prime}(b)+\sqrt{A k} N_{2}(b)
\end{array}\right]\left[\begin{array}{c}
u(a) \\
u(b)
\end{array}\right]=\left[\begin{array}{c}
-A u^{\prime}(a)-\sqrt{A k} u(a) \\
A u^{\prime}(b)+\sqrt{A k} u(b)
\end{array}\right]
$$

que teniendo en cuenta que $N_{1}(a)=1, N_{1}(b)=0, N_{2}(a)=0, N_{2}(b)=1$ queda reducida a

$$
\left[\begin{array}{cc}
-A N_{1}^{\prime}(a)-\sqrt{A k} & -A N_{2}^{\prime}(a) \\
A N_{1}^{\prime}(b) & A N_{2}^{\prime}(b)+\sqrt{A k}
\end{array}\right]\left[\begin{array}{l}
u(a) \\
u(b)
\end{array}\right]=\left[\begin{array}{c}
-A u^{\prime}(a)-\sqrt{A k} u(a) \\
A u^{\prime}(b)+\sqrt{A k} u(b)
\end{array}\right]
$$

Como puede observarse la información contenida en ambas ecuaciones de equilibrio es la misma, pero la naturaleza de la matriz de rigidez y de lasacciones nodalesde equilibrio es diferente. Por ejemplo, la matriz de rigidez en el primer caso es regular pues $\widetilde{u}^{t} \widetilde{K} \tilde{u}=\int_{a}^{b}\left(A u^{\prime 2}+k u^{2}\right) d x>0, u \in N(L), \widetilde{u}^{t} \neq(0,0)$ $y$, en el segundo caso, es singular, pues $\tilde{u}^{t} \tilde{K} \tilde{u}=\int_{a}^{b}\left(L_{1}(u)\right)^{2} d x=0, u \in N(L)$, para algunos $\tilde{u}^{t} \neq(0,0)$, por ejemplo para $u(a), u(b)$ correspondientes a $u=\exp (-\sqrt{k / A} x)$.

Asimismo las acciones nodales de equilibrio en el primer caso son los axiles del cálculo de estructuras. Para el segundo caso, sin embargo, el significado de las acciones nodales es distinto al usual. La ecuación de equilibrio global en el caso factorizado contiene exactamente la misma información que la correspondiente al operador autoadjunto en la forma usual y se podria, con las modificaciones convenientes de la matriz de rigidez global y del vector de acciones nodales, transformar una cualquiera de las ecuaciones en la otra.

Esta cuestión del estudio comparativo de ambos planteamientos ha sido objeto de estudio en algunos trabajos sobre splines. Por ejemplo, en la referencia [39] se analiza la construcción de splines en tensión utilizando directamente la minimización en el espacio de Sobolev $H^{2}[a, b]$ de los funcionales cuadráticos derivados de las formas bilineales

$$
a(f, g)=\int_{a}^{b}\left(f^{\prime \prime} g^{\prime \prime}+c^{2} f^{\prime} g^{\prime}\right) d t, b(f, g)=\int_{a}^{b}\left(f^{\prime \prime}+c f^{\prime}\right)\left(g^{\prime \prime}+c g^{\prime}\right) d t^{-}
$$


4.6 Comentarios sobre la interpretación estructural en los splines usuales y aplicación de una extensión de la idea de spline generalizado

La idea de spline desde sus origenes ha ido, generalmente, asociada al hecho de que dichas funciones minimizan un cierto funcional de energía que puede ser interpretado desde un punto de vista fisico o geométrico. Los ejemplos más notables en este sentido son los splines cúbicos y los splines en tensión y variantes de ambos tipos. Hay gran número de referencias que tratan sobre la construcción de tipos particulares de splines a partir de la minimización directa de ciertos funcionales de energía, de tal manera que pequeñas modificaciones en el funcional han servido para introducir nuevos tipos de splines, los cuales pueden considerarse como simples variantes de los splines cúbicos y de los splines en tensión. Una referencia que consideramos de gran interés, precisamente por analizar desde el punto de vista variacional esta evolución de los diferentes tipos de splines, es el trabajo de Hagen y Schulze ([46]). A continuación y con objeto de ilustrar la evolución que hemos indicado, comentamos la interpretación estructural de algunos tipos particulares de splines e indicamos la posible aplicación del enfoque matricial propuesto en este trabajo.

NOTA: No comentamos aqui los clásicos trabajos de Larkin ([55]), Glass ([56]), Woodford ([57]) y Malcolm ([58]), pues, aunque todos ellos tratan el interesante problema de la interpolación de datos mediante la teoría de la elástica, el planteamiento que siguen es no lineal y, por tanto, no interpretable en la forma matricial usual correspondiente al enfoque lineal del método de los desplazamientos.

En primer lugar y obviando los detalles, por bien conocidos, para los splines cúbicos y los splines en tensión usuales $([38,40,43,44,45])$, centramos nuestra discusión en los $v$-splines que son introducidos por Nielson ([39]) con el propósito de conseguir resultados análogos a los splines en tensión, en lo referente a la eliminación de puntos de inflexión extraños, pero empleando funciones polinómicas cúbicas. La idea de Nielson es sustituir el segundo sumando del funcional de energía de deformación de los splines en tensión

$$
\int_{a}^{b}\left(f^{\prime \prime}(t)\right)^{2} d t+c^{2} \int_{a}^{b}\left(f^{\prime}(t)\right)^{2} d t
$$

por la expresión $\sum_{i=1}^{n-1} v_{i}\left(f^{\prime}\left(t_{i}\right)\right)^{2}, v_{i} \geq 0$ donde $a=t_{0}<t_{1}<. .<t_{n}=b$. Posteriormente Foley ([45]) introduce otra modificación en el funcional de energía de los splines en tensión sustituyendo el primer sumando por $\sum_{i=0}^{n-1} \omega_{i} \int_{i}^{i+1}\left(f^{\prime \prime}(t)\right)^{2} d t, \omega_{i}>0$ de esta forma el funcional que emplea tiene un número mayor de pesos que pueden modificarse a voluntad. El funcional que queda finalmente es

$$
\sum_{i=0}^{n-1} \omega_{i} \int_{i}^{t+1}\left(f^{n}(t)\right)^{2} d t+\sum_{i=1}^{n-1} v_{i}\left(f^{\prime}\left(t_{i}\right)\right)^{2}
$$

Por otra parte, el problema a resolver es determinar en el conjunto de funciones

$$
F=\left\{f \in H^{2}, f\left(t_{i}\right)=y_{i}, i=0, . ., n, f^{\prime}\left(t_{0}\right)=y_{0}^{\prime}, f^{\prime}\left(t_{n}\right)=y_{n}^{\prime}\right.
$$

aquéllas que mimimizan los funcionales indicados, llegándose a que dichas funciones son, en ambos casos, splines cúbicos en el sentido generalizado, es decir, splines cúbicos de la clase $C^{\mathrm{l}}$.

Ni en la publicación de Nielson ni en la de Foley hay interpretaciones de tipo estructural que indiquen que por ejemplo, en el primer caso, la función extremal representa el modelo de una viga continua de rigidez constante apoyada en cada uno de los nodos intermedios con empotramientos en los extremos y con empotramientos elásticos en todos los nodos intermedios. Por otra parte, en el segundo caso, la rigidez es además constante en cada subintervalo. La resolución, desde nuestro planteamiento matricial, es inmediata. En primer lugar, la función que se busca no es más que un spline cúbico en sentido generalizado, donde, con nuestra notación $L(u)=D^{2}\left(A(x) D^{2} u\right)$, donde la rigidez es constante en cada subintervalo, es decir $A(x)=\omega_{i}, x \in\left(x_{i}, x_{i+1}\right), i=1, . ., n-1$. El funcional de energía potencial total es, en este caso,

$$
F(g)=\frac{1}{2} \sum_{i=1}^{n-1} \omega_{i} \int_{x_{1}}^{x_{n}}\left(g^{\prime \prime}(x)\right)^{2} d x-\sum_{i=2}^{n-1} g^{\prime}\left(x_{i}\right) M_{i}
$$


que los vectores de desplazamientos y de fuerzas nodales son aquí

$$
\mathbf{u}=\left(u_{1}^{*}, u_{1}^{\prime *}, u_{2}^{*}, u_{2}^{\prime}, . ., u_{n-1}^{*}, u_{n-1}^{\prime} u_{n}^{*}, u_{n}^{\prime *}\right), \mathbf{Q}=\left(F_{1}, M_{1}, F_{2}, M_{2}^{*}, . ., F_{n-1}, M_{n-1}^{*}, F_{n}, M_{n}\right)
$$

donde se ha indicado con un (*) los valores conocidos. Se han distinguido en el vector de acciones nodales los dos tipos de acciones que actúan en cada nodo, esto es, las duales de los desplazamientos verticales y las duales de las pendientes. Como puede verse las pendientes en los nodos intermedios son desconocidas; pues bien, basta expresar, en este caso, sus acciones duales como conocidas. Estas acciones, en términos estructurales, son los momentos en los nodos intermedios $y$, como en dichos nodos hay realmente empotramientos elásticos, se tiene que $M_{i}=-k_{i} u_{i}^{\prime}, i=2, . ., n-1$. Obsérvese que el funcional de energia quedaria en la forma

$$
F(g)=\frac{1}{2}\left(\sum_{i=1}^{n-1} \omega_{i} \int_{x_{1}}^{x_{n}}\left(g^{\prime \prime}(x)\right)^{2} d x+\sum_{i=2}^{n-1} k_{i}\left(g^{\prime}\left(x_{i}\right)\right)^{2}\right)
$$

El método matricial propuesto en este trabajo, en lugar de minimizar el funcional anterior para el conjunto dado de condiciones de interpolación, como es usual en las publicaciones ya citadas, consiste simplemente en añadir a los elementos de la diagonal principal de la matriz de rigidez global del spline generalizado, los valores $k_{i}$ en las posiciones correspondientes a $u_{i}^{\prime}, i=2, . ., n-1$, tomando el nuevo vector de acciones nodales justamente el valor nulo para las componentes situadas en las posiciones citadas. Esta operación, como puede comprobarse, es equivalente a la minimización del funcional anterior y, además, tiene la ventaja de que podemos imponer diferentes condiciones de interpolación en cada nodo y modificarlas a voluntad en cada caso, sin necesidad de tener que construir el sistema de ecuaciones para cada conjunto particular de condiciones.

Hay que indicar que además de introducir estas modificaciones en el funcional de los splines en tensión para conseguir reducir el número de puntos de inflexión extraños que aparecen para determinados conjuntos de datos cuando se emplean los splines cúbicos usuales, se han realizado modificaciones más simples, como la que resulta de eliminar el segundo sumando de (4.16). En definitiva, la modificación consiste en trabajar únicamente con splines cúbicos con peso, eligiendo dichos pesos $\omega_{i}$ convenientemente. Un trabajo muy notable,por los resultados que se obtienen, es el de Salkauskas ([40]) donde se toma como peso, en cada subintervalo, una cierta función de la pendiente de cada tramo $\omega_{i}=\left\{1+\left(y_{i+1}-y_{i}\right)^{2} / h_{i}^{2}\right\}^{-3}, h_{i}=x_{i+1}-x_{i}$. Otra alternativa propuesta por de Boor ([6, p. 303]) para el mismo propósito es utilizar splines cúbicos ordinarios con nudos adicionales y los correspondientes datos de ordenadas en ellos, situados en las zonas donde se quiere que la gráfica de la función, modifique la curvatura o cambie de dirección. Pues bien, para estos planteamientos, a pesar de que el objetivo tiene una importante interpretación estructural, los métodos utilizados en ambas publicaciones han sido fundamentalmente de tipo algebraico.

Otro caso de interés donde puede explicitarse además la idea de acciones nodales generadas por uniones de tipo elástico, es el de los splines de suavizado al que de Boor en A Practical Guide to Splines ([6]) dedica un capitulo completo. El funcional que se desea minimizar en el espacio $H^{m}\left[x_{1}, x_{n}\right]$ es de la forma

$$
p \sum_{i=1}^{n} k_{i}\left(f\left(x_{i}\right)-y_{i}\right)^{2}+(1-p) \int_{x_{1}}^{x_{n}}\left(f^{(m)}(x)\right)^{2} d x, \quad 0<p<1
$$

Para este caso el procedimiento de construcción que proponemos se basa también en una simple modificación de la ecuación de equilibrio global correspondiente a los splines polinómicos generalizados de grado $2 m-1$ correspondientes al operador diferencial $L(u)=D^{m}\left(A(x) D^{m} u\right)$ donde la función que hace las veces de la rigidez (en un sentido generalizado) puede tomarse constante en cada subintervalo, generalizando algo más el funcional anterior.

La interpretación estructural consiste en considerar que los puntos de la gráfica de la función que se busca, están unidos elásticamente en cada nodo a los puntos deordenada $y_{i}$, con constantes de valor

$p k_{i}$. Esto, como puede verse observando el funcional de energía después de desarrollar-el primer sumando, equivale a una unión elástica en cada nodo, de la gráfica, a un punto de ordenada nula (es 
decir, en el eje $\mathrm{x}$ ) y simultáneamente una acción nodal de valor $p k_{i} y_{i}$ que es dual del desplazamiento en el nodo. En resumen, la ecuación de equilibrio global de los splines de grado $2 m-1$ con peso, donde $A(x)=A_{i}($ cte $), x \in\left(x_{i}, x_{i+1}\right), i=1, . ., n-1$ se modifica en la forma siguiente: a los elementos situados en la diagonal principal de la matriz de rigidez global, en las posiciones correspondientes a los desplazamientos $u_{i}, i=1, . ., n$ se le suma el valor de la constante elástica $p k_{i}$, y el vector de acciones nodales tiene todas sus componentes nulas excepto aquéllas que tienen la misma posición que los desplazamientos, las cuales tienen por valor $p k_{i} y_{i}$.

Como puede deducirse a partir de la estructura del vector de acciones nodales, en este caso, la regularidad de la función spline que se obtiene es de la clase $C^{2 m-2}$ cuando se toman todos los pesos iguales, es decir si $A(x)=1-p, x \in\left[x_{1}, x_{n}\right]$, en caso contrario la regularidad se reduce a $C^{m-1}$ en todos aquellos nodos donde haya discontinuidad en el peso.

El caso particular de estos splines de suavizado para $m=2$, al corresponder de nuevo a un caso de splines cúbicos ha sido tambien muy estudiado. En la referencia [59, p. 57] se comenta, con diversas ilustraciones gráficas, la influencia del parámetro $p$ y se indican, a su vez, otras referencias que tratan sobre la determinación automática de dicho parámetro.

Otra aplicación del enfoque matricial propuesto es la construcción de splines paramétricos tanto en el plano como en el espacio, es decir para $(x(t), y(t))$ en $\mathbf{R}^{2} \circ(x(t), y(t), z(t))$ en $\mathbf{R}^{3}$. En este caso se trata de construir una curva con $n$ nodos que se corresponden con puntos $P_{i}, i=1, . ., n$ del plano o del espacio y el parámetro $t$ puede ser de tipo uniforme: en los sucesivos nodos va tomando los valores $t_{i}=0,1, . ., n-1$, o representar la longitud acumulada de la cuerda: el parámetro aproxima la longitud de arco de la curva, mediante la longitud de la poligonal que une los puntos, es decir $t_{1}=0, t_{i+1}=t_{i}+\left|\overline{P_{i} P_{i+1}}\right|, i=1, . ., n-1$. Para estos splines paramétricos se establece una ecuación de equilibrio tanto local, en cada $\left[t_{i}, t_{i+1}\right]$ como global, en $\left[t_{1}, t_{n}\right]$ para cada una de las componentes por separado, considerando cada componente como una función spline, donde la variable independiente es el parámetro $t$. Al ser las matrices de rigidez local y global las mismas para las distintas componentes, cabe la posibilidad de emplear una única ecuación de equilibrio tomando el vector de desplazamientos y de acciones nodales de modo que incluya las componentes de cada una de las funciones. Aqui, si cabe, la aplicación del método, es más interesante desde el punto de vista de las interpretaciones de tipo estructural, al no ser éstas tan directas como en los splines generalizados (no paramétricos).

A continuación realizamos unos breves comentarios sobre otras alternativas posibles al método matricial de cálculo de splines generalizados propuesto en este trabajo. El método desarrollado aquí es el método de los desplazamientos donde, en primer lugar, se determinan los desplazamientos desconocidos y después se pueden determinar tanto las acciones nodales exteriores desconocidas en los nodos correspondientes, asi como las acciones nodales de equilibrio en los extremos de cada elemento. Una alternativa al método propuesto, podría ser, manteniendo la analogía estructural, el método de compatibilidad o de las fuerzas, donde las incógnitas que se determinarian en primer lugar son las acciones nodales de equilibrio en los extremos de cada elemento y después los movimientos de los nodos.

Este método de cálculo de los splines generalizados basado, en la idea de compatibilidad de desplazamientos, es muy sencillo de construir a partir del paralelismo citado, de ahí que omitamos su desarrollo. Por otra parte hay esquemas de cálculo de splines basados fundamentalmente en enfoques de tipo algebraico, que, en el fondo y de una manera parcial, están aplicando la idea citada de compatibilidad. Esto sucede cuando se eligen como incógnitas derivadas altas (por ejemplo, desde la de orden $m$ hasta

la de orden $2 m-1$ ) que, como hemos visto, van asociadas, desde nuestra interpretación, a acciones nodales, y se imponen después condiciones de compatibilidad de desplazamientos en los nodos. Un ejemplo de esto se tiene cuando se determinan splines cúbicos eligiendo como incógnitas las derivadas segundas en los nodos, imponiendo después, como condiciones de compatibilidad, la igualdad de derivadas primeras por la izquierda y derecha en cada uno de los nodos intermedios, obteniendo. las conocidas ecuaciones de los tres momentos. 
Respecto a la ventaja de un procedimiento frente al otro, proponemos, sin profundizar en la discusión, como método más sistemático para la construcción de splines (formación de la ecuación de equilibrio global), el de desplazamientos, aunque para casos muy particulares donde, por ejemplo, las condiciones de interpolación no van a variar, la idea de compatibilidad puede ofrecer alguna ventaja. A este respecto puede ser ilustrativa, aunque no de traslación inmediata a los splines generalizados, la discusión realizada en $[60$, p. 226].

Finalizamos este apartado apuntando, de manera esquemática, la posibilidad de construir splines cuyo operador diferencial $L_{1}$ (autoadjunto o no) es de orden $m$, mediante una cierta extensión de la teoría de splines generalizados asociados al operador $L=L_{1}^{*} L_{1}$ que es autoadjunto y de orden $2 m$. La idea consiste en sumergir dicho spline dentro de los splines generalizados relativos al operador $L=L_{1}^{*} L_{1}$, imponiendo para este último solamente las condiciones de regularidad correspondientes al spline asociado al operador $L_{1}$. El procedimiento de construcción se basa en utilizar las ecuaciones de equilibrio local correspondientes a los splines asociados al operador $L$, ensambladas de manera que el nuevo spline generalizado no sea obligatoriamente de la clase $C^{m-1}$. Esto se consigue desacoplando convenientemente ciertas derivadas en el vector de desplazamientos e imponiendo, además, ciertas condiciones de nulidad para las componentes del vector de acciones nodales que son duales de las componentes incógnitas del vector de desplazamientos.

Para ilustrar el esquema anteriormente citado, nos vamos a centrar simplemente en la determinación de los splines de segundo grado mediante los splines de grado cinco, considerando éstos en un sentido extendido. Este caso, a pesar de su sencillez, permite,con una sencilla generalización, dar la construcción sistemática de los splines de grado par. Dichos splines van asociados a operadores diferenciales que no son autoadjuntos $y$, por dicho motivo, los métodos de construcción empleados para tales splines son fundamentalmente algebraicos ([1, p. 109]). Los splines de segundo grado van asociados al operador diferencial $L_{1}(u)=D^{3} u$ y los de quinto grado a $L(u)=L_{1}^{*}\left(L_{1}(u)\right)=-D^{6} u$. Sean los nodos $x_{1}<x_{2}<. .<x_{n}$, imponiendo que los splines de segundo grado sean funciones de la clase $C^{1}$, desacoplando en el ensamblado de las ecuaciones de equilibrio de los splines de quinto grado las derivadas segundas en los nodos intermedios, esto es, se considerarán para dichos nodos

$u_{i}^{\prime \prime}=u^{\prime \prime}\left(x_{i}^{-}\right), u_{i}^{\prime \prime}=u^{\prime \prime}\left(x_{i}^{+}\right), i=2, . ., n-1$ como valores distintos. De esta forma el vector de desplazamientos de la ecuación de equilibrio global para los splines de quinto grado desde este punto de vista extendido (funciones de la clase $C^{1}$ en lugar de ser $C^{2}$ ) es

$$
\mathbf{u}^{t}=\left(u_{1}, u_{1}^{\prime}, u_{1}^{\prime \prime}, u_{2}, u_{2}^{\prime}, u_{2}^{n-} u_{2}^{n+}, u_{3}, u_{3}^{\prime}, u_{3}^{n-}, u_{3}^{n+}, . ., u_{n}, u_{n}^{\prime}, u_{n}^{\prime \prime}\right)
$$

Asimismo el correspondiente vector 'de acciones nodales es

$$
\mathrm{Q}^{t}=\left(-u_{1}^{(5)}, u_{1}^{(4)},-u_{1}^{(3)}, u_{2}^{(5)-}-u_{2}^{(5)+},-u_{2}^{(4)-}+u_{2}^{(4)+}, u_{2}^{(3)-},-u_{2}^{(3)+}, . ., u_{n}^{(5)},-u_{n}^{(4)}, u_{n}^{(3)}\right)
$$

La matriz de rigidez global para este nuevo spline de quinto grado se obtiene de ensamblar las matrices de rigidez (4.6) en la forma usual, teniendo en cuenta que para los subintervalos que van desde el segundo hasta el final, las matrices de rigidez indicadas aumentan en uno la dimensión como resultado de intercalar una fila y una columna de ceros entre la segunda y tercera. Asimismo todas las componentes del vector de acciones nodales que se corresponden con desplazamientos no fijados se deben hacer nulas.

Como ejemplo numérico supongamos que los nodos son $x_{i}=i-1, i=1, . ., 4$, si los datos conocidos

son por ejemplo $\mathbf{u}^{\mathbf{t}}=(2,-,-, 1,-,-,-, 1.5,1,-,-, 2.5,-,-)$ que corresponden, en este caso, a fijar la ordenada en todos los nodos y la pendiente de valor unidad en el nodo tercero y fijamos, como hemos indicado, las acciones nodales con valor nulo para las componentes duales de los desplazamientos incógnitas, la resolución del correspondiente sistema proporciona los valores desconocidos. Estos valores son $\mathbf{u}^{\mathbf{t}}=\left(*,-2,2,{ }^{*}, 0,2,1, *, *, 1,0, *, 1,0\right)$.

El spline de segundo grado que resulta es

$$
x^{2}-2 x+2, x \in[0,1] \quad(x-1)^{2} / 2+1, x \in(1,2] \quad(x-2)+3 / 2, x \in(2,3]
$$




\section{Solución nodal exacta y acciones equivalentes en problemas de contorno unidimensionales}

Desarrollamos en este punto la aplicación de los splines generalizados a la resolución de problemas de contomo unidimensionales. La idea utilizada consiste en emplear en el método usual de elementos finitos de Galerkin ([32, p. 116] y [61, p. 35]) funciones de prueba y funciones test o de ponderación pertenecientes al espacio de dimensión finita engendrado por los splines generalizados correspondientes al operador $L$ de orden $2 m$ de la ecuación diferencial. Esta elección del espacio de funciones conduce a soluciones que son exactas en los nodos. Es más, dicho resultado se mantiene aunque se tome un espacio arbitrario para las funciones de prueba. Por otra parte, la idea de acción equivalente (ya considerada en [66]) sugiere una nueva aproximación, para la solución de los problemas de contorno, que además de interpolar en los nodos los valores exactos de los desplazamientos, interpola también los valores de las acciones de equilibro. Esta nueva solución puede interpretarse como un cierto spline generalizado de orden $4 m$ asociado al operador $L^{2}$.

\subsection{Solución nodal exacta en problemas de contorno unidimensionales}

El problema de contorno que se plantea en este apartado y cuya definición precisa se da después, consiste en resolver, en forma aproximada, una ecuación diferencial ordinaria autoadjunta no homogénea en el intervalo $\left[x_{1}, x_{n}\right]$, de modo que la solución en los nodos $x_{1}<x_{2}<. .<x_{i}<$.. $<x_{n}$ verifica ciertas condiciones de contomo, tanto de tipo esencial como de tipo natural, relativas al operador diferencial de la ecuación. Estas condiciones deben garantizar la unicidad de solución del correspondiente problema de contomo de $n$ puntos.

Sea la ecuación diferencial no homogénea

$$
L(u)=f
$$

definida en $(a, b)$, siendo el operador diferencial $L$ el definido por (2.5). Multiplicando por la función $\nu$ ambos miembros de (5.1) e integrando por partes (residuos ponderados) y aplicando (2.9) se obtiene la expresión

$$
\int_{a}^{b} \sum_{j=0}^{m} a_{j} D^{j} u D^{j} v d x=\int_{a}^{b} f v d x+\sum_{i=1}^{2 m} l_{i}(v) \bar{l}_{i}(u)
$$

la cual corresponde a la formulación débil de (5.1) o, lo que es lo mismo, al principio de los trabajos virtuales.

A continuación pasamos a construir la ecuación de equilibrio en $[a, b]$ para las soluciones de (5.1). Damos pasos análogos a los efectuados en la demostración del resultado (IV) para lo que suponemos que el operador diferencial $L$ y la longitud del intervalo genérico $[a, b]$ verifican las mismas hipótesis del apartado 4.1. Asimismo suponemos que $f$ es continua en $(a, b)$ y que existen y son finitos los limites siguientes: $\lim _{x \rightarrow c} f(x), c=a^{+}, b^{-}$.

La solución de (5.1) que verifica las condiciones de interpolación indicadas en el citado apartado es

$$
u=w+u_{H}=w+\sum_{i=1}^{2 m} r_{i} N_{i}
$$

donde $w$ es una solución de la ecuación completa que verifica las condiciones homogéneas siguientes $l_{i}(w)=0, i=1, . ., 2 m$. Estas condiciones de interpolación siempre pueden imponerse ya que si $\bar{w}$ es una solución particular arbitraria, entonces $w=\bar{w}-\sum_{i=1}^{2 m} l_{i}(\bar{w}) N_{i}$ verifica, tal y como puede comprobarse, las condiciones citadas. Obsérvese que se está suponiendo que el problema de contorno definido por (5.1) y las condiciones de contorno $l_{i}(u)=r_{i}, i=1, . ., 2 m$, siendo $r_{i}$ números reales arbitrarios dados, posee como solución única a la función dada por (5.3). 


$$
a(u, v)=\int_{i}^{b} \sum_{j=0}^{m} a_{j} D^{j} u D^{j} v d x=\sum_{i=1}^{2 m} l_{i}(u) \bar{l}_{i}(v)+\int_{a}^{b} L(v) \dot{u} d x
$$

y considerando ahora en el primer miembro de la expresión (5.2) la $u$ dada por (5.3) y $v=N_{j}$ se obtiene para cada $j=1, . ., 2 m$

$$
\sum_{i=1}^{2 m} l_{i}\left(w+\sum_{k=1}^{2 m} r_{k} N_{k}\right) \bar{l}_{i}\left(N_{j}\right)=\int_{i}^{b} f N_{j} d x+\sum_{i=1}^{2 m} l_{i}\left(N_{j}\right) \bar{l}_{i}(u)
$$

donde se ha tenido en cuenta que $L\left(N_{j}\right)=0$. Simplificando queda

$$
\sum_{i=1}^{2 m} k_{j i} r_{i}=\int_{d}^{b} f N_{j} d x+\bar{l}_{j}(u)
$$

Expresando lo anterior en forma matricial se tiene la ecuación

o equivalentemente mediante la expresión particionada

$$
\widetilde{K} \tilde{u}=\tilde{f}+\widetilde{q}^{c}
$$

$$
\left[\begin{array}{ll}
K_{11}^{(a, b)} & K_{12}^{(a, b)} \\
K_{21}^{(a, b)} & K_{22}^{(a, b)}
\end{array}\right]\left[\begin{array}{l}
\tilde{u}\left(a^{+}\right) \\
\tilde{u}\left(b^{-}\right)
\end{array}\right]=\left[\begin{array}{l}
\tilde{f}\left(a^{+}\right) \\
\tilde{f}\left(b^{-}\right)
\end{array}\right]+\left[\begin{array}{l}
\tilde{q}^{c}\left(a^{+}\right) \\
\tilde{q}^{c}\left(b^{-}\right)
\end{array}\right]
$$

que es verificada por cualquier solución de (5.1) en el intervalo $(a, b)$. Dicha ecuación la denominamos de equilibrio local en el intervalo $[a, b]$, donde la matriz de rigidez $\widetilde{K}$ es la misma matriz que en (4.1), el vector de desplazamientos nodales es análogamente a (4.2) $\tilde{u}=\widetilde{r}$, el vector de cargas nodales de equilibrio o de contorno $\widetilde{q}^{C}$ está definido como en (4.2), con la salvedad de que la función $u$ es alli solución de la ecuación diferencial homogénea mientras que aqui lo es de la ecuación completa (5.1) y finalmente el vector $\widetilde{f}$ que denominamos de cargas nodales equivalentes es

$$
\tilde{f}^{t}=\left(\int_{b}^{b} f N_{1} d x, . . . \int_{a}^{b} f N_{2 m} d x\right)
$$

Las componentes del vector de cargas nodales equivalentes verifican que

$$
\begin{aligned}
& \int_{d}^{b} f N_{j} d x=-\bar{l}_{j}(w) \\
& j=1, . .2 m
\end{aligned}
$$

Resultado que se deduce trivialmente de (5.5) o equivalentemente de (5.6) al considerar la solución particular $u=w$, es decir $r_{1}=. .=r_{2 m}=0$. Lo anterior permite poner

$$
\sum_{i=1}^{2 m} k_{j i} r_{i}=\bar{l}_{j}(u-w)=\bar{l}_{j}\left(u_{H}\right)
$$

que como puede verse corresponde a la ecuación de equilibrio (4.1) del elemento de spline generalizado que interpola los mismos datos que la solución de la ecuación completa (5.1), teniéndose $\widetilde{q}=\widetilde{f}+\widetilde{q}^{C}$.

De los resultados anteriores se deriva la siguiente interpretación de tipo estructural: las cargas nodales equivalentes, correspondientes a una acción repartida, son las opuestas de las cargas nodales de equilibrio relativas a la solución particular de la ecuación completa que verifica condiciones de interpolación homogéneas. Esta es la idea que, en cálculo de estructuras mediante el proceso de empotramiento en los nudos y aplicando el principio de superposición, permite pasar de cargas repartidas a cargas concentradas únicamente en los nudos. A partir de aquí se llega, de forma un tanto intuitiva, al hecho de que los movimientos de los nudos en la estructura con cargas repartidas y los de la que tiene únicamente cargas concentradas (las correspondientes) en dichos nudos, son los mismos.

Veamos ahora que se llega a la misma ecuación de equilibrio local (5.6) sustituyendo, en el primer miembro de (5.2), la solución exacta $u$ de (5.1) por cualquier aproximación $\hat{u}$ tal que

$$
\begin{aligned}
& l_{i}(u)=l_{i}(\hat{u})=r_{i} \\
& i=1, . ., 2 m
\end{aligned}
$$


En efecto, de (5.4) resulta $a(u, v)=a(\hat{u}, v)=\sum_{i=1}^{2 m} r_{i} \bar{l}_{i}(v)$ ya que la función de ponderación $v$ es tal que $L(v)=0$. De esta forma tomando $v=N_{j}$ se tiene para cada $j=1, . ., 2 m$

$$
a\left(u, N_{j}\right)=a\left(\hat{u}, N_{j}\right)=\sum_{i=1}^{2 m} k_{j i} r_{i}
$$

Obsérvese que la aproximación $\hat{u}$ dada por $\hat{u}=\sum_{i=1}^{2 m} r_{i} \hat{N}_{i}$ con $l_{i}\left(\hat{N}_{j}\right)=\delta_{i j}$ puede ser totalmente arbitraria. Naturalmente una aproximación de particular interés es aquélla donde la base de Lagrange de funciones de interpolación está formada por soluciones de la ecuación homogénea asociada a (5.1), es $\operatorname{decir} \hat{N}_{i}=N_{i}, i=1, . .2 m$.

La expresión (5.7) resulta también de forma inmediata poniendo $v=\sum_{i=1}^{2 m} s_{i} N_{i}$ con $s_{i}$ arbitrarios, asi (5.2) queda en la forma

$$
\widetilde{s}^{t} \widetilde{K} \tilde{r}=\widetilde{s}^{t}\left(\tilde{f}+\widetilde{q}^{c}\right)
$$

y teniendo en cuenta que $\widetilde{s}^{t}=\left(s_{1}, . ., s_{2 m}\right)$ es arbitrario se obtiene la expresión indicada. Empleando para (5.8) la expresión matricial particionada en términos de $u$ y $v$ ésta queda como

$$
\left(\widetilde{v}\left(a^{+}\right)^{t}, \tilde{v}\left(b^{-}\right)^{t}\right)\left[\begin{array}{ll}
K_{11}^{(a, b)} & K_{12}^{(a, b)} \\
K_{21}^{(a, b)} & K_{22}^{(a, b)}
\end{array}\right]\left[\begin{array}{l}
\tilde{u}\left(a^{+}\right) \\
\tilde{u}\left(b^{-}\right)
\end{array}\right]=\left(\widetilde{v}\left(a^{+}\right)^{t}, \tilde{v}\left(b^{-}\right)^{t}\right)\left(\left[\begin{array}{c}
\tilde{f}\left(a^{+}\right) \\
\tilde{f}\left(b^{-}\right)
\end{array}\right]+\left[\begin{array}{l}
\tilde{q}^{c}\left(a^{+}\right) \\
\widetilde{q}^{c}\left(b^{-}\right)
\end{array}\right]\right)
$$

Por otra parte el resultado de reciprocidad indicado en (2.13) para dos funciones $u$ y $v$ que verifican en el intervalo genérico $(a, b), L(u)=f, L(v)=g$, se puede expresar con la notación de este apartado en la forma

$$
\widetilde{v}^{t} \widetilde{q}_{u}^{c}+\int_{a}^{b} f v d x=\tilde{u}^{t} \widetilde{q}_{v}^{c}+\int_{a}^{b} g u d x
$$

La interpretación mecánica o estructural de la expresión anterior es inmediata: el trabajo de las acciones correspondientes a la solución $u$ realizado sobre los desplazamientos relativos a la solución $v$ es igual al trabajo de las acciones correspondientes a $v$ sobre los desplazamientos dados por $u$.

A continuación se obtiene, de manera análoga a los splines generalizados mediante el proceso usual de ensamblado (véase el apartado 4.3), la ecuación de equilibrio global en el intervalo $\left[x_{1}, x_{n}\right]$ que verifica cualquier función $u(x)$ solución de

$$
L(u)=f \text { en } \bigcup_{i=1}^{n-1}\left(x_{i}, x_{i+1}\right)
$$

que sea de la clase $C^{m-1}$ en dicho intervalo $\left[x_{1}, x_{n}\right]$.

Sumando las expresiones (5.10) correspondientes a cada intervalo $\left(x_{i}, x_{i+1}\right), i=1, . ., n-1$ y considerando por la regularidad citada que $\tilde{u}\left(x_{i}^{+}\right)=\widetilde{u}\left(x_{i}^{-}\right)$en los nodos internos y lo mismo para $v, y$ poniendo como en el caso de los splines generalizados

$$
\begin{gathered}
\mathbf{u}^{\mathrm{t}=}\left(\tilde{u}\left(x_{1}\right)^{t}, . ., \tilde{u}\left(x_{n}\right)^{t}\right)=\left(u\left(x_{1}\right), . ., u^{(m-1)}\left(x_{1}\right), . ., u\left(x_{n}\right), . ., u^{(m-1)}\left(x_{n}\right)\right)= \\
=\left(r_{11}, . ., r_{1 m}, . ., r_{n 1}, . ., r_{n m}\right)
\end{gathered}
$$

e idénticamente para $v$, se obtiene

$$
\mathbf{v}^{t} \mathbf{K u}=\mathbf{v}^{\mathbf{t}}\left(\mathbf{F}+\mathbf{Q}^{\mathrm{c}}\right)
$$

la cual,teniendo en cuenta que se verifica para cualquier $\mathbf{v}$, es equivalente a esta otra que denominamos ecuación de equilibrio global para las soluciones de (5.12) que verifican la condicion de regularidad indicada 
La matriz $\mathbf{K}$ que denominamos de rigidez global es la misma que en (4.9), sin embargo el vector columna $\mathbf{F}$ que denominamos de acciones nodales equivalentes es aqui el que resulta de ensamblar las acciones nodales equivalentes correspondientes a cada elemento, asimismo el vector $\mathbf{Q}^{\mathbf{C}}$ de acciones nodales exteriores es, como en los splines generalizados, el resultado de ensamblar las acciones nodales de equilibrio locales. Este último vector es como (4.11), con la salvedad de que aquí está definido para funciones que son solución de la ecuación completa (5.12) en lugar de venir dado para splines generalizados, de ahí que lo distingamos, como en el caso local, con el superíndice c. Los vectores $\mathbf{F}$ y $\mathbf{Q}^{\mathrm{C}}$ pueden expresarse como

$$
\begin{aligned}
\mathbf{F}^{\mathbf{t}} & =\left(f_{11}, . ., f_{1 m}, . ., f_{n 1}, . ., f_{n m}\right) \\
\left(\mathbf{Q}^{\mathrm{C}}\right)^{\mathbf{t}} & =\left(\bar{l}_{11}(u), . ., \bar{l}_{1 m}(u), . ., \bar{l}_{n 1}(u), . ., \bar{l}_{n m}(u)\right)
\end{aligned}
$$

donde las componentes del vector de cargas nodales equivaientes son

$$
f_{i j}=\int_{x_{1}}^{x_{n}} f N_{i j}(x) d x, i=1, . ., n, j=1, . ., m
$$

siendo $N_{i j}(x)$ las funciones de forma globales definidas en $\left[x_{1}, x_{n}\right]$ que constituyen la base de Lagrange de splines generalizados, es decir $l_{p q}\left(N_{i j}\right)=\delta_{i p} \delta_{j q}, p=1, . ., n, q=1, . ., m$ donde las formas lineales de interpolación $l_{p q}$ vienen definidas como $l_{p q}(u)=u^{(q-1)}\left(x_{p}\right)$ y $L\left(N_{i j}\right)=0$ en $\bigcup_{i=1}^{n-1}\left(x_{i}, x_{i+1}\right)$. Asimismo las formas lineales $\bar{l}_{i j}$ son las componentes del vector definido en (4.11) a partir de los operadores dados en (2.7).

Por otra parte, análogamente al caso local $\mathbf{Q}=\mathbf{F}+\mathbf{Q}^{\mathbf{C}}$ es el vector de acciones nodales exteriores correspondiente al spline generalizado que interpola en los nodos $x_{1}, . ., x_{n}$ los mismos valores que la solución de (5.12) considerada.

Señalamos que, como en el caso de los splines generalizados, la expresión (5.13) es una identidad la cual únicamente pone de manifiesto que fijados los desplazamientos mediante el vector $\mathbf{u}$ y fijadas las acciones exteriores $f$ en $\left[x_{1}, x_{n}\right]$, se tienen de manera univoca las acciones nodales exteriores $\mathbf{Q}^{\mathbf{C}}$.

La expresión análoga a la (5.2) correspondiente a la formulación débil para $\left[x_{1}, x_{n}\right]$ se obtiene sumando cada una de las que resultan en (5.2) al considerar los subintervalos $\left(x_{i}, x_{i+1}\right), i=1, . ., n-1$, resultando

$$
\int_{x_{1}}^{x_{n}} \sum_{j=0}^{m} a_{j} D^{j} u D^{j} v d x=\int_{x_{1}}^{x_{n}} f v d x+\mathbf{v}^{\mathbf{t}} \mathbf{Q}^{\mathbf{C}}
$$

De aqui se obtiene de manera directa la ecuación de equilibrio (5.13) expresando $v$ mediante las funciones de forma globales de splines generalizados $N_{i j}(x)$.

También se tiene, de manera análoga a (5.11) el resultado de reciprocidad para las soluciones $u, v$ de (5.12) correspondientes a las acciones $f, g$, y que interpolan los desplazamientos dados por los vectores $\mathbf{u}, \mathbf{v}$. En efecto, basta sumar las expresiones análogas a la (5.11) correspondientes a los subintervalos $\left(x_{i}, x_{i+1}\right), i=1, . ., n-1$ obteniéndose

$$
\mathbf{v}^{\mathbf{t}} \mathbf{Q}_{u}^{\mathrm{C}}+\int_{x_{1}}^{x_{n}} f v d x=\mathbf{u}^{\mathrm{t}} \mathbf{Q}_{v}^{\mathrm{C}}+\int_{x_{1}}^{x_{n}} g u d x
$$

De esta expresión resulta también, de forma inmediata, la ecuación de equilibrio global (5.13ł Basta para ello aplicar (5.15) entre la solución de (5.12) que interpola los datos dados por el vector de desplazamientos $\mathbf{u}$ y las funciones de forma globales ya indicadas. 
Podemos definir ahora en $\left[x_{1}, x_{n}\right]$ un problema de contorno de $n$ puntos donde la solución $u$, además de cumplir (5.12) verifica las condiciones esenciales de interpolación del tipo Hermite-Birkoff

$$
\begin{aligned}
l_{i j}(u)=u^{(j-1)}\left(x_{i}\right)=r_{i j},(i, j) \in I_{n} \times I_{m} & =\{1, . ., n\} \times\{1, . ., m\} \text { donde } \\
r_{i j} & =r_{i j}^{*},(i, j) \in J_{p}
\end{aligned}
$$

son datos, siendo $J_{p}$ un cierto subconjunto de $I_{n} \times I_{m}$ de cardinal $p$. Asimismo la solución verifica las condiciones naturales $\bar{l}_{i j}(u)=Q_{i j}^{C},(i, j) \in I_{n} \times I_{m}$ de modo que

$$
Q_{i j}^{C}=Q_{i j}^{C^{*}},(i, j) \in J_{s}
$$

son tambien datos, siendo $J_{s}=I_{n} \times I_{m}-J_{p}$ el conjunto de cardinal $s=n \times m-p$. En definitiva se está indicando, del mismo modo que en el apartado 4.4, que se conocen $p$ componentes de las $n \times m$ del vector $\mathbf{u}$ de desplazamientos nodales y $s=n \times m-p$ componentes del vector $\mathbf{Q}^{\mathrm{C}}$ del vector de acciones nodales exteriores.

El problema de contorno planteado se puede expresar de manera equivalente en la forma siguiente: Obtener la solución $u \in C^{m-1}\left[x_{1}, x_{n}\right]$ de la ecuación diferencial

$$
L(u)=\bar{f}=f+\sum_{(i, j) \in J_{s}} Q_{i j}^{C^{*}}(-1)^{j-1} \delta^{(j-1)}\left(x-x_{i}\right)
$$

que verifica las condiciones de interpolación dadas por (5.16), donde $\bar{f}$ es la acción generalizada que mediante el simbolismo de las distribuciones hemos expresado en función de derivadas de deltas de Dirac. Cuando la acción $f$ sea nula en todos los subintervalos, la solución exacta será un spline generalizado,pues, en dicho caso, $\bar{f}$ se compone únicamente de acciones nodales exteriores.

NOTA: Obsérvese que aunque $u$ pertenece al espacio de funciones indicado es en realidad un elemento de $H^{m}$ y aunque $f \in C\left(\bigcup_{i=1}^{n-1}\left(x_{i}, x_{i+1}\right)\right), \bar{f}$ pertenece a $H^{-m}\left[x_{1}, x_{n}\right]$, es decir, al dual de $H^{m}\left[x_{1}, x_{n}\right]$. De hecho, desde el punto de vista de las aplicaciones, los casos más interesantes son aquéllos donde la acción repartida $f$ es realmente de $H^{-m}$ en cada subintervalo $\left(x_{i}, x_{i+1}\right)$, siendo la solución $u$ en dicha situación una función de $H^{m} \subset C^{m-1}$ en cada subintervalo. Nótese que en este caso más general la solución dejaría de ser de la clase $C^{2 m}$ en los citados subintervalos y, por tanto, las derivadas que definen a los operadores diferenciales (2.7) deben ser consideradas en el sentido de las distribuciones.

Teniendo en cuenta que todas las componentes del vector $\mathbf{F}$ de acciones nodales equivalentes son conocidas y que $\mathbf{Q}=\mathbf{F}+\mathbf{Q}^{\mathbf{C}}$ representa las acciones nodales exteriores para el spline generalizado que interpola los mismos datos que la solución que se busca, el problema de unicidad de solución para el problema de contomo planteado queda reducido al problema de interpolación con splines generalizados abordado en el apartado citado, por lo que nos remitimos a lo alli expresado, incluyendo el resultado (VI).

Veamos ahora que la resolución de este problema por el método de Galerkin conduce a valores nodales exactos, obteniéndose en consecuencia los mismos resultados que $P$. Tong ([22]), con la particularidad de que aqui no exigimos que la forma bilineal $a(u, v)$ sea definida positiva. Asimismo se demuestra que la exactitud nodal se consigue, aunque el espacio de funciones de prueba no sea de splines generalizados.

Sea $V$ el espacio de dimensión infinita $V=\left\{h \in H^{m}\left[x_{1}, x_{n}\right] \forall(i, j) \in J_{p}, h^{(j-1)}\left(x_{i}\right)=0\right\}$ teniendo en cuenta la formulación equivalente para el problema de contorno:

Obtener $u \in g+V$ tal que $a(u, v)=l(v), \forall v \in V$ donde $a(u, v)$ es el primer miembro de (5.14) y

$$
l(v)=\int_{\alpha_{1}}^{x_{n}} \bar{f} v d x=\int_{x_{1}}^{x_{n}} f v d x+\sum_{(i, j) \in J_{s}} Q_{i j}^{C^{*}} v^{(j-1)}\left(x_{i}\right)
$$


Introduciendo ahora en dicha ecuación la aproximación $\hat{u}=g+\sum_{(i, j) \in J_{s}} r_{i j} \hat{N}_{i j} \in g+S_{h}, S_{h} \subset V$ donde $g=\sum_{(i, j) \in J_{p}} r_{i j}^{*} \hat{N}_{i j}$ es una función que interpola los desplazamientos datos, expresada mediante las funciones de forma globales $\hat{N}_{i j}$ (arbitrarias) donde $l_{p q}\left(\hat{N}_{i j}\right)=\delta_{i p} \delta_{j q}, p=1, . ., n, q=1, . ., m$. y ponderando con los elementos del subespacio $V_{h}$ de $V$ engendrado por los splines generalizados $N_{p q}, \forall(p, q) \in J_{s}$, se obtiene el sistema de ecuaciones

$$
\sum_{(i, j) \in J_{p}} r_{i j}^{*} a\left(\hat{N}_{i j}, N_{p q}\right)+\sum_{(i, j) \in J_{s}} r_{i j} a\left(\hat{N}_{i j}, N_{p q}\right)=\int_{x_{1}}^{x_{n}} f N_{p q} d x+Q_{p q}^{C^{*}}, \forall(p, q) \in J_{s}
$$

Esta expresión es,por los resultados ya vistos para las ecuaciones de equilibrio local y teniendo en cuenta el ensamblado, la misma que se obtiene para la solución exacta. La expresión que resulta después de reordenar convenientemente como en el apartado 4.4 y llamar $\mathbf{u}_{p}^{*}, \mathbf{u}_{\mathrm{s}}$ a los desplazamientos datos $\mathrm{e}$ incógnitas respectivamente y $\mathbf{Q}_{s}^{\mathbf{C}^{*}}$ a las acciones nodales datos, es justamente la segunda línea de (4.12), es decir $\mathbf{K}_{s s} \mathbf{u}_{s}=\mathbf{F}_{s}+\mathbf{Q}_{\mathbf{s}}^{\mathrm{C}^{*}}-\mathbf{K}_{\mathrm{sp}} \mathbf{u}_{\mathrm{p}}^{*}=\mathbf{Q}_{\mathbf{s}}^{*}-\mathbf{K}_{\mathrm{sp}} \mathbf{u}_{\mathrm{p}}^{*}$. Obsérvese que el único requerimiento para la resolución del problema de contorno es que la matriz $\mathbf{K}_{\mathrm{ss}}$ sea regular y no necesariamente definida positiva.

Se tiene el siguiente resultado:

(IX) En el supuesto de que la solución exacta del citado problema de contorno de $n$ puntos sea única ( $\mathrm{K}_{\mathrm{ss}}$ regular), la solución aproximada obtenida por el método de Galerkin en la forma indicada (aproximación arbitraria de la solución y ponderación con splines generalizados) toma en los nodos los mismos valores que la solución exacta. Además, una vez determinados los desplazamientos nodales desconocidos, se puede determinar en cada subintervalo los valores exactos de las acciones nodales de equilibrio a partir de la correspondiente ecuaciórı de equilibrio local.

En las aplicaciones lo usual es tomar las funciones $\hat{N}_{i j}=N_{i j}, i=1, . ., n, j=1, . ., m$ de esta manera la solución aproximada $\hat{u}$ del problema de contorno será también un spline generalizado, no obstante consideramos también la posibilidad de otras aproximaciones que están relacionadas con la idea de acción (repartida) equivalente.

Otro problema, diferente al de contorno de $n$ puntos citado, y que describimos esquemáticamente, consiste en determinar los valores de los desplazamientos en todos los nodos $x_{1}, x_{2}, . ., x_{n}$ y las acciones de equilibrio por la izquierda y derecha en dichos nodos, conociendo para el primer nodo $x_{1}$ (o cualquier otro nodo) los valores del vector de desplazamientos $\widetilde{u}\left(x_{1}^{+}\right) y$, simultáneamente, las acciones locales de equilibrio $\widetilde{q}\left(x_{1}^{+}\right)$y asimismo la acción $f$ en el intervalo $\left[x_{1}, x_{n}\right]$ y las acciones nodales exteriores en todos los nodos.

Este problema, análogo a los de valor inicial (obsérvese que los datos de las derivadas en el nodo $x_{1}$, desde la de orden $m$ hasta la de orden $2 m-1$, se dan también, aunque de forma un tanto implícita mediante las componentes de $\widetilde{q}\left(x_{1}^{+}\right)$), se resuelve de forma exacta y de manera recurrente a partir de las expresiónes deducidas de la ecuación (5.8), teniéndose el siguiente esquema de cálculo

$$
\begin{gathered}
\tilde{u}\left(x_{i+1}^{+}\right)=\tilde{u}\left(x_{i+1}^{-}\right)=\left(K_{12}^{i}\right)^{-1}\left(\tilde{f}\left(x_{i}^{+}\right)+\widetilde{q}^{C}\left(x_{i}^{+}\right)-K_{11}^{i} \tilde{u}\left(x_{i}^{+}\right)\right) \\
\widetilde{q}^{C}\left(x_{i+1}^{+}\right)=\left(\mathbf{Q}^{\mathrm{C}}\right)_{i+1}-\widetilde{q}^{C}\left(x_{i+1}^{-}\right)=\left(\mathbf{Q}^{\mathrm{C}}\right)_{i+1}+\widetilde{f}\left(x_{i+1}^{-}\right)-K_{21}^{i} \widetilde{u}\left(x_{i}^{+}\right)-K_{22}^{i} \widetilde{u}\left(x_{i+1}^{-}\right)
\end{gathered}
$$


donde $\left(\mathbf{Q}^{\mathbf{C}}\right)_{i+1}^{t}=\left(Q_{i+1,1}^{C}, . ., Q_{i+1, m}^{C}\right)$ representa la acción nodal exterior en el nodo $x_{i+1}$. Obsérvese que para su aplicación únicamente se requiere la regularidad de la submatriz $K_{12}^{i}$. En el apartado 6.3 se desarrolla una aplicación de este caso a un problema dinámico.

\subsection{Acciones equivalentes en problemas de contorno unidimensionales}

Considerando en la ecuación de equilibrio en $[a, b]$ correspondiente a (5.1) el vector de cargas nodales equivalentes, puede observarse que fijados los desplazamientos $\tilde{u}=\widetilde{r}$, hay infinitos estados de carga diferentes a $f$ que proporcionan las mismas acciones nodales de equilibrio $\widetilde{q}^{C}$. En efecto, basta considerar otra carga cualquiera $g$ que en relación con $f$ verifique $\int_{a}^{b}(f-g) N_{i} d x=0, i=1, . ., 2 m$, pues, de este modo, los valores que intervienen en la ecuación de equilibrio son únicamente $\int_{h}^{b} f N_{i} d x, i=1, . ., 2 m$ y, consecuentemente, las acciones de equilibrio correspondientes a $f$ y $g$ son las mismas. Diremos que en dicho caso las dos acciones o estados de carga son equivalentes.

La condición anterior la podemos expresar asi: dos estados de carga definidos por las funciones $f$ y $g$ son equivalentes en $(a, b)$ si su diferencia $f-g$ es ortogonal a $N(L)$, es decir, al espacio de funciones engendrado por las soluciones de la ecuación homogénea $L(u)=0$ en dicho intervalo.

Un caso de especial interés, por los resultados que se obtienen y las interpretaciones que permite realizar, es precisamente aquél donde la acción equivalente a una acción $f$ dada se toma en el espacio de funciones $N(L)$. Dicha acción (repartida) equivalente, al pertenecer a un espacio de dimensión finita podemos considerarla como un cierto estado simplificado de carga, además ésta tiene gran regularidad, pues en lugar de ser continua como la carga de partida (o incluso en la situación más general de $H^{-m}$ ), es $C^{2 m}(a, b)$. Dicha acción, que representamos por $F$, es la proyección ortogonal de la acción dada $f$ sobre el núcleo del operador diferencial y podemos considerarla como un representante cánonico de la clase de equivalencia a la que pertenece la acción de partida. La expresión de la carga $F$ en la base de funciones de forma locales se obtiene a partir del teorema de la proyección ortogonal como

$$
F=\left(N_{1}, . ., N_{2 m}\right) G^{-1} \widetilde{f}
$$

donde los elementos de la matriz de Gram $G$ son $\int_{i}^{b} N_{i} N_{j} d x, i, j=1, . ., 2 m$

Una consecuencia interesante derivada de la elección de $F$ como representante del estado de carga consiste en considerar la relación entre las soluciones de $L(u)=f$ y $L(U)=F$ donde las condiciones de contorno esenciales para ambos casos son las mismas: $l_{i}(u)=l_{i}(U)=r_{i}, i=1, . ., 2 m$. Resulta de lo anterior que las acciones nodales de equilibrio coinciden, es decir, $\bar{l}_{i}(u)=\bar{l}_{i}(U), i=1, . ., 2 m$, y como $F \in N(L)$ se tiene además que $U$ pertenece al núcleo del operador $L^{2}$, esto es, $L^{2}(U)=0$ (suponemos para esto que los coeficientes del operador $L$ tienen regularidad suficiente para que $U$ sea una función de la clase $\left.C^{4 m}(a, b)\right)$. Obsérvese que la función $U$ puede interpretarse como la solución de $L^{2}(U)=0$ que interpola los $4 m$ valores siguientes: $l_{i}(u), \bar{l}_{i}(u), i=1, . ., 2 m$. La solución $U$ puede calcularse sin determinar $F$ pues basta obtener el elemento del núcleo de $L^{2}$ que interpola los $4 m$ valores indicados. A posteriori puede determinarse, si se quiere, $F$, mediante $F=L(U)$ o por (5.18).

Todo lo anterior podemos resumirlo del siguiente modo: 
(X) Dada la acción o estado de carga $f$ en $(a, b)$ existe otro estado $F$ que pertenece al espacio de soluciones de la ecuación homogénea, y que denominamos estado de carga simplificado, que genera las mismas cargas nodales equivalentes que $f$, siendo $F$ la proyección ortogonal de $f$ en el citado espacio. Además, la solución de $L(U)=F$ que satisface $l_{i}(U)=l_{i}(u)$, $i=1, . ., 2 m$, verifica que $\bar{l}_{i}(U)=\bar{l}_{i}(u), i=1, . ., 2 m$ y puede interpretarse por tanto como la solución de $L^{2}(U)=0$ que interpola los valores $l_{i}(u), \bar{l}_{i}(u), i=1, . ., 2 m$.

Los resultados anteriores se extienden de manera inmediata a todo el dominio. En efecto, la equivalencia de acciones en todo el dominio puede definirse del siguiente modo: dos acciones son equivalentes en todo el dominio si restringidas a cada subintervalo resultan equivalentes. Por otra parte,si una acción se sustituye por otra equivalente en todo el dominio, es decir, si $f$ se sustituye en cada subintervalo por cualquier otra equivalente $g$, las ecuaciones de equilibrio local no se modifican y, consecuentemente, la ecuación de equilibrio global (5.13) es la misma. Se concluye que las soluciones de los problemas de contorno de $n$ puntos (u otros, como, por ejemplo, el ya mencionado de valor inicial) cuando existan y sean únicas, serán las mismas cuando las acciones sean equivalentes, es decir, los desplazamientos nodales desconocidos y las acciones de equilibrio en cada intervalo coincidirán.

Es interesante señalar por otra parte que las soluciones del problema de contorno de $n$ puntos mediante el spline generalizado y mediante la solución interpoladora $U$ correspondiente a la carga repartida equivalente $F$, representan, de alguna manera, situaciones extremas en relación con la distribución de la carga. En éfecto, en el primer caso lo que se hace es transformar el problema en otro donde únicamente hay acciones nodales exteriores (las iniciales más las nodales equivalentes) y ninguna acción repartida y en el segundo la acción inicial se transforma en una acción repartida con cierto grado de regularidad.

Por otro lado, el hecho de que para acciones equivalentes las acciones nodales de equilibrio coincidan en los extremos de cada subintervalo permite inferir una cierta relación en el interior de dichos subintervalos entre los valores de los operadores de (2.7), $P_{i}, i=0, . ., m-1$, para dos soluciones correspondientes a acciones equivalentes. Dicha relación la determinamos a continuación para las soluciones correspondientes a las acciones $f$ y $F$. Integrando por partes en el intervalo genérico, después de multiplicar por la función $v \in N(L)$ ambos miembros de $L(u)=f$ y $L(U)=F$ y teniendo en cuenta los operadores definidos en (2.7), se obtienen las expresiones siguientes

$$
\begin{aligned}
& -\sum_{i=0}^{k}\left[\left(P_{i}(u)-P_{i}(U)\right) v^{(i)}\right]_{a}^{b}+\int_{a}^{b}\left[\left(\sum_{i=0}^{k} a_{i} D^{i}(u-U) D^{i} v\right)+\left(P_{k}(u)-P_{k}(U)\right) v^{(k+1)}\right] d x=0 \\
& k=0, . ., m-1
\end{aligned}
$$

las cuales, considerando (2.11) y la propiedad de interpolación $\bar{l}_{i}(U)=\bar{l}_{i}(u), i=1, . ., 2 m$, queda reducida únicamente al segundo sumando, es decir

$$
\begin{aligned}
& \int_{a}^{b}\left[\left(\sum_{i=0}^{k} a_{i} D^{i}(u-U) D^{i} v\right)+\left(P_{k}(u)-P_{k}(U)\right) v^{(k+1)}\right] d x=0 \\
& k=0, . ., m-1
\end{aligned}
$$

La expresión anterior para el caso $k=m-1$ y considerando (2.7) y (2.8) equivale a $a(u-U, v)=0$ donde hay que tener en cuenta que $U \in N\left(L^{2}\right)$ y $v \in N(L)$.

En la aplicación que se realiza del concepto de acción equivalente en el apartado 6.3 se determina la aproximación $U$ mediante interpolación con funciones del núcleo del operador $L^{2}$. También en la aplicación que se realizó de dicho concepto, a la aproximación de desplazamientos y esfuerzos en la viga de Bernoulli-Euler ([66]), se empleó dicho procedimiento para el cálculo de la aproximación $U$. En dicho trabajo se puso de manifiesto la bondad de resultados no sólo de los desplazamientos sino tamtién de los esfuerzos. Precisamente en relación con la aproximación de los esfuerzos, se obtuvieron,en el citado trabajo, las correspondientes expresiones particulares de (5.19) que permitieron dar una justificación sobre la aproximación del momento flector y del esfuerzo cortante en el interior de cada elemento de viga. 


\subsection{Comentarios}

Todo el desarrollo realizado en este punto en relación con la exactitud nodal, de las soluciones aproximadas de problemas de contorno unidimensionales, se ha basado en la consideración de que el operador diferencial de $L$ de orden $2 m$ es autoadjunto. Cabe, sin embargo, la posibilidad de aplicar gran parte de las ideas aqui expuestas a otras situaciones donde el operador no sea autoadjunto. En dicho caso puede comprobarse que se consigue también exactitud nodal utilizando funciones de ponderación pertenecientes al núcleo del operador adjunto $L^{*}$. Otra posibilidad, en cierta manera equivalente, consiste en transformar la ecuación $L(u)=f$ en otra $L(U)=F$ donde $F$ se obtiene como resultado de proyectar $f$ sobre el núcleo de $L^{*}$ y si la regularidad del operador lo permite transformar la ecuación inicial en $L^{*} L(U)=0$ y considerar $U$ como un cierto spline generalizado relativo al operador $L^{*} L$ que es autoadjunto. Algunos de estos aspectos son comentados en un apéndice, que hace referencia al trabajo de P. Tong ([22]), en el texto de Zienkiewicz y Taylor ([62,p. 631]) aunque desde un enfoque ajeno al de los splines generalizados.

\section{Aplicaciones}

En este punto se exponen diversas aplicaciones del método de cálculo de splines generalizados a problemas de diseño gráfico, cálculo de vigas y cálculo dinámico.

\subsection{Un ejemplo de aplicación gráfica de los splines generalizados de orden seis}

A continuación se describe una aplicación gráfica de la teoría desarrollada empleando splines generalizados de orden seis, los cuales incluyen como caso particular a los splines de quinto grado.

El problema planteado para ilustrar las posibilidades del enfoque propuesto, consiste en determinar una función spline $u(x)$ de orden seis en el intervalo $[0,17]$ con nodos $x_{i}=0,5,9,13,17$, que verifica distintas condiciones de interpolación en cada nodo. Así, en el primer nodo, $x_{1}=0$, se dan como datos la ordenada, primera derivada y segunda derivada, siendo los valores $u=4, u^{\prime}=-2.7, u^{\prime \prime}=7.9563$. En el segundo nodo, $x_{2}=5$, sólo se fija el valor de la primera derivada, donde $u^{\prime}=0$. En el tercer nodo, $x_{3}=9$, se da el valor de la primera y segunda derivada, $u^{\prime}=0.5, u^{\prime \prime}=0.0931$. En el nodo cuarto, $x_{4}=13$, la ordenada y segunda derivada, con valores $u=6$ y $u^{\prime \prime}=0$. Finalmente, en el nodo quinto, $x_{5}=17$, se dan como datos, de manera análoga al primer nodo, los valores $u=8, u^{\prime}=-0.7 \mathrm{y}$ $u^{\prime \prime}=0.9093$.

Los datos y condiciones de interpolación anteriores vienen motivados por el propósito de diseñar un enlace en $S$ entre las circunferencias de radios 3 y 2 respectivamente, que aparecen dibujadas en la figura 1. Los puntos de tangencia del enlace con las circunferencias citadas se consideran datos y son el $(0,4)$ y $(17,8)$, con pendientes en dichos puntos de -2.7 y -0.7 . Asimismo, los valores de las derivadas segundas correspondientes a las curvaturas $1 / 3$ y $1 / 2$, para las pendientes dadas, son respectivamente $u^{\prime \prime}=7.9563$ y $u^{\prime \prime}=0.9093$. Por otra parte, el enlace debe cumplir los requerimientos adicionales de que la pendiente en el punto de abscisa 5 sea nula, la pendiente y radio de curvatura del enlace en el punto de abscisa 9 tomen respectivamente los valores de 0.5 y 15 y, finalmente, que el enlace pase por el punto de coordenadas $(13,6)$ y tenga curvatura nula en dicho punto.

Entre los diferentes tipos de splines generalizados de orden seis hemos elegido, para esta aplicación, los asociados al operador diferencial

$$
L(u)=(-1)^{3} D^{3}\left(a_{3}(x) D^{3} u\right)+(-1)^{2} D^{2}\left(a_{2}(x) D^{2} u\right)=-A u^{(6)}+B u^{(4)}
$$

donde las funciones $a_{3}(x)=A>0$ y $a_{2}(x)=B \geq 0$ son constantes en cada subintervalo $(a, b)$. El spline, para el caso $B>0$, está definido en cada subintervalo mediante una combinación lineal de las funciones que componen el vector $\tilde{x}^{t}=\left(1, z, z^{2}, z^{3}, \exp (z \sqrt{B / A}), \exp (-z \sqrt{B / A})\right), z=x-a$, y que se obtienen a partir de las raíces de la ecuación característica $r^{4}\left(-A r^{2}+B\right)=0$. Cuando 
$B=0$, resultan los splines de quinto grado con peso, siendo, en dicho caso, $\widetilde{x}^{t}=\left(1, z, z^{2}, z^{3}, z^{4}, z^{5}\right)$. La construcción de los splines se realiza mediante la formulación matricial desarrollada en el punto 4 . Así la ecuación de equilibrio local en cada subintervalo se obtiene de forma análoga a los ejemplos desarrollados en el apartado 4.2, teniendo en cuenta que los operadores $P_{i}, i=0,1,2$,definidos en (2.7) son, en este caso, $P_{0}(u)=A u^{(5)}-B u^{(3)}, P_{1}(u)=-A u^{(4)}+B u^{(2)}, P_{2}(u)=A u^{(3)}$. Por otra parte la ecuación de equilibrio global para el spline en el intervalo $[0,17]$ se calcula en la forma indicada en el apartado 4.3, teniendo en cuenta las contribuciones de las ecuaciones locales relativas a los subintervalos $\left[x_{i}, x_{i+1}\right] i=1, . ., 4$. Finalmente consideramos en la ecuación global los desplazamientos que son datos y las acciones nodales datos, es decir, las duales de los desplazamientos desconocidos. En esta aplicación el vector de desplazamientos $\mathbf{u}$ de 15 componentes $\left(u, u^{\prime}, u^{\prime \prime}\right.$ en cada nodo), es el mismo para todos los casos (a) a (e), viniendo indicadas en negrita en las tablas de la figura 1 aquéllas que son datos. De la misma manera se indican en negrita las componentes datos del vector $\mathbf{Q}$ de acciones nodales. Los restantes valores (de estilo normal) de $\mathbf{u}$ y $\mathbf{Q}$, se obtienen a partir de la resolución del sistema que se deriva de la ecuación global una vez considerados los datos.

Con los casos indicados en la figura 1 con las letras (a) a (e), se pretende poner también de manifiesto, la influencia que tiene en la gráfica del spline, la modificación del valor de los pesos $A$ y $B$ en los distintos subintervalos y la modificación de los valores de las acciones nodales. Así, en el primer grupo de splines, casos (a), (b) y (c), las acciones nodales correspondientes a las componentes no prefijadas del vector de desplazamientos (las indicadas en negrita en el vector $\mathbf{Q}$ ), se han fijado todas a cero. De esta manera la modificación de la gráfica es debida,únicamente, a la variación de los pesos en los distintos subintervalos.

El caso (a) dónde los pesos son $A=1, B=0$ en todos los subintervalos, corresponde al de un spline de quinto grado y como puede verse, la gráfica de la curva de enlace, tiene poca suavidad, debido a los cambios de signo en la curvatura que ocurren en diversos puntos, aparte del de abscisa $x=13$, donde la condición de curvatura nula ha sido impuesta. Dichas oscilaciones se evitan aumentando el valor de $B$ respecto al de $A$ (véanse los pesos en la tabla adjunta al gráfico superior de la figura 1), lo que provoca una disminución en promedio de la curvatura,tal y como se pone de manifiesto en las gráficas de (b) y (c). En estos casos, al ser las acciones nodales citadas nulas, el spline es, de entre todas las funciones que verifican los datos prefijados del vector de desplazamientos, aquélla que hace mínimo el funcional de energía de deformación

$$
F(g)=\frac{1}{2} \int_{0}^{7}\left[A\left(g^{(3)}\right)^{2}+B\left(g^{(2)}\right)^{2}\right] d x
$$

Ahora realizamos una comparación entre los casos (c), (d) y (e), considerando, para los dos últimos, la presencia de acciones nodales no nulas y manteniendo los mismos pesos que en (c) (véase el gráfico inferior y tablas adjuntas al mismo en la figura 1). El propósito es que, manteniendo la suavidad del enlace proporcionado por (c), conseguir que la curva no corte a la circunferencia de radio 2. Para ello y procediendo por tanteos, modificamos el valor de la acción nodal dual de la pendiente en $x=13$, pasando ésta a tener el valor de 5.0 (caso (d)). Como esta modificación desplaza la gráfica hacia abajo más de lo deseado, se cambia también el valor de la acción nodal dual del desplazamiento en $x=5$, asignando a ésta un nuevo valor de 0.5 ,obteniendo la gráfica que se indica en el caso (e). Esta última la consideramos, sin entrar en la justificación de detalles analíticos, como una solución aceptable para el problema del enlace. En los casos (d) y (e) los splines, en lugar de minimizar (6.2), minimizan la energia potencial total, la cual, para (d) es $F(g)-5.0 g^{\prime}(13)$ y para (e) $F(g)-0.5 g(5)-5.0 g^{\prime}(13)$.

Es evidente que se obtendrian los mismos resultados añadiendo como nuevos datos en el vector de desplazamientos, el valor modificado de la pendiente en $x=13$ y del desplazamiento en $x=5$. Sin embargo, esta forma de proceder llevaria aparejada la modificación de la matriz del sistema a resolver, resultando más cómodo cambiar los valores de las acciones nodales, los cuales únicamente requieren modificar el término independiente del sistema.

Por otra parte indicamos que splines como el propuesto en esta aplicación y otros análogos como, por ejemplo, los asociados al operador $L(u)=-A u^{(6)}+B u^{(4)}-C u^{(2)}$ pueden ser una buena alternativa, desde el punto de vista gráfico, a los bien estudiados splines cúbicos con peso y splines en tensión, pues, sobre éstos, tienen la gran ventaja de que el grado de regularidad es $C^{2}$, además de incluir mayor número de parámetros, lo que les da también una mayor flexibilidad. 

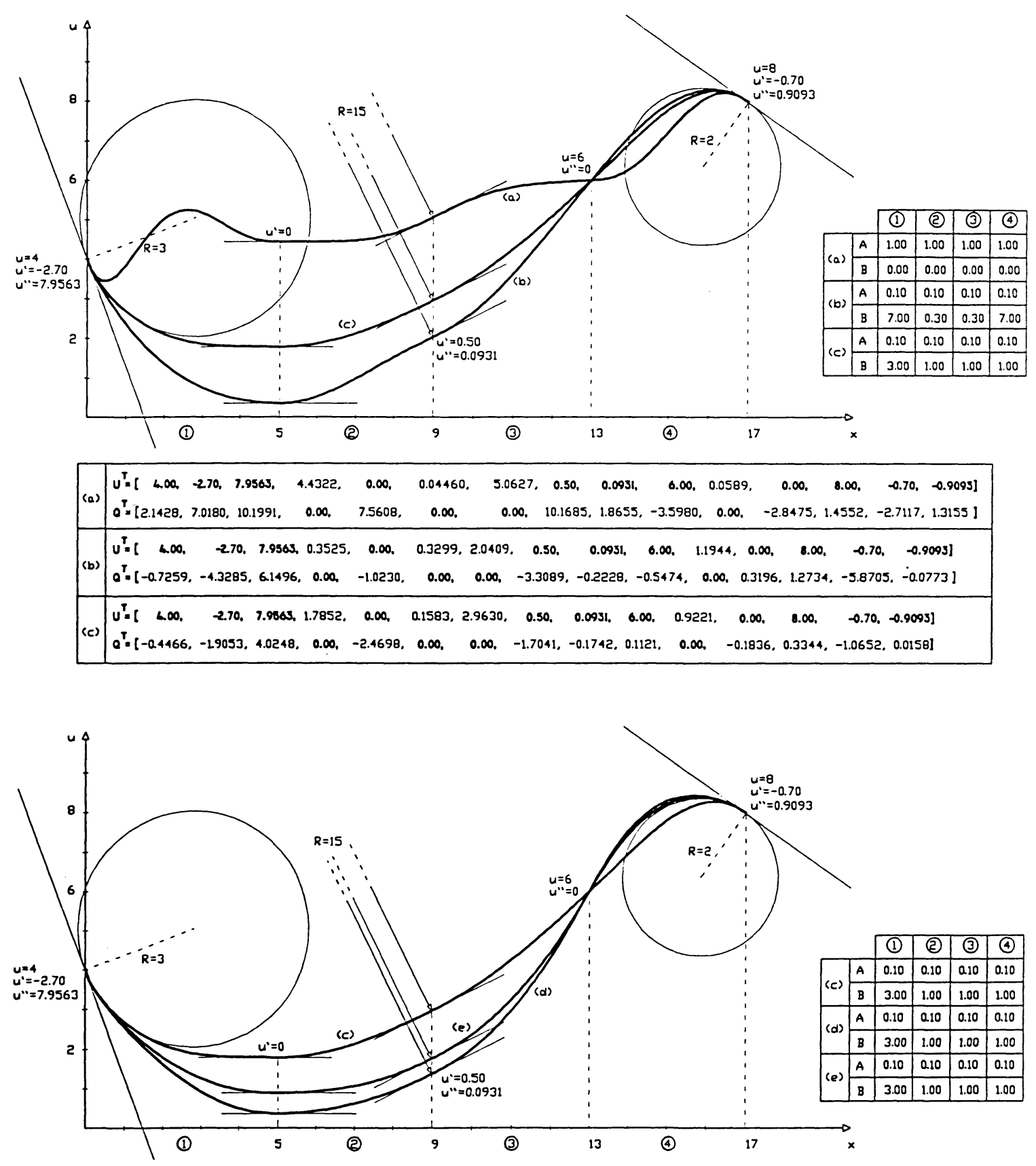

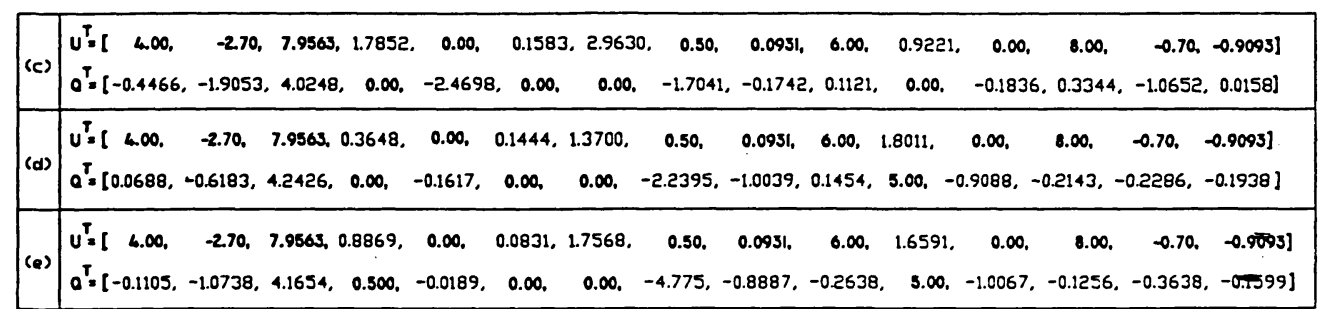


Finalmente se indica que si no se emplean herramientas de cálculo simbólico para el manejo de las funciones trascendentes puede haber una cierta limitación en la utilización de splines generalizados en aplicaciones como la anterior, cuando se pretende conseguir una regularidad alta en la función de interpolación. Una alternativa, desde el punto de vista práctico, que resuelve en parte esta dificultad consiste en emplear espacios de elementos finitos de polinomios a trozos, que aproximen al spline generalizado asociado al correspondiente operador diferencial. Este procedimiento, que es la base de los métodos energéticos de modelización geométrica, se describe, entre otros, en el interesante trabajo de Cardona y Samartín ([63]).

NOTA: Debe observarse que el problema geométrico del enlace entre las dos circunferencias no es, en si mismo, el objetivo de este apartado ya que con él sólo se pretende motivar una aplicación geométrica que ilustre las diferentes posibilidades de la metodología desarrollada para el cálculo de splines. En este sentido, precisamos que si el problema del enlace correspondiese, por ejemplo, al trazado en planta de una via de comunicación, las soluciones dadas por los diferentes splines calculados, deberian ser tomadas como primeras aproximaciones, pues una solución ingenieril del mismo, requiere, entre otras cuestiones, que la ecuación de la curva no dependa del sistema de referencia. Esto, como es bien conocido, puede plantearse utilizando la concatenación de arcos de curvas definidas por sus ecuaciones intrinsecas, lo que conduce, por otro lado, a la resolución de problemas no lineales de cierta complejidad. Las referencias [64] y [65] constituyen ejemplos representativos de este enfoque no lineal del problema de trazado, sobre todo la primera, donde se aborda, con gran detalle, el interesante problema de la determinación de los splines clotoidales. La segunda referencia, por su parte, aborda los clásicos y más elementales problemas de determinación de curvas de enlace entre recta y circunferencia, en las distintas situaciones posibles, empleando, según los casos, una o dos clotoides como curvas de transición.

\subsection{Cálculo de una viga continua en un medio elástico de Winkler sometida simultáneamente a acciones axiles y flexión}

En este punto se expone, una aplicación de los resultados del apartado 5.1 al cálculo, por elementos finitos, de los desplazamientos y las acciones nodales de equilibrio en un problema de contorno no homogéneo de $n$ puntos correspondiente a una ecuación diferencial de cuarto orden que modeliza el comportamiento estructural de una viga continua sobre un medio elástico de Winkler sometida a flexión y simultáneamente a cargas axiles. El método de cálculo tiene la particularidad de que los valores determinados, en cada uno de los nodos, son exactos. Por otra parte, el objetivo de este apartado es análogo al de los trabajos de análisis de vigas sobre fundación elástica indicados en ias referencias siguientes [48,49,50,51], con la singularidad de que, aqui, se incluyen además cargas axiles y también, y de mayor interés desde nuestro punto de vista, que la metodologia empleada procede de una simple particularización del planteamiento matricial desarrollado para los splines generalizados, lo que permite un tratamiento más sistemático de estos problemas.

Para la aplicación se ha tomado una viga continua de tres tramos alineados, donde los dos primeros tramos de 8 y $6 m$ respectivamente, tienen una sección de $0.5 \times 0.5 m$ y el tercero de $10 m$, una sección de $0.5 \times 0.8 \mathrm{~m}$. El módulo de elasticidad del material se considera con un valor de $E=23.000 \mathrm{~N} / \mathrm{mm}^{2}$ y el coeficiente de balasto que caracteriza la respuesta del terreno, de $k=20.000 \mathrm{KN} / \mathrm{m}^{3}$. Asimismo, la viga se considera sujeta mediante una serie de coacciones que limitan determinados desplazamientos en los puntos de apoyo, de forma que el desplazamiento vertical se supone nulo mientras que los giros en dichos apoyos son libres, manteniendo la continuidad de la viga. Por su parte, para la adecuada transmisión del axil, el desplazamiento horizontal no se considera coaccionado, salvo en la unión de los tramos segundo y tercero, donde, para mantener el equilibrio horizontal,se impide la traslación del apoyo.

Cada tramo de viga está sometido a un conjunto de acciones o estados de carga de modo que la ecuación diferencial que rige cada uno de dichos tramos es diferente. Así, exceptuando la consideración común del peso propio y la respuesta del terreno, el primer tramo de viga está sometido a una acción repartida y constante de $100 \mathrm{KN} / \mathrm{m}$ y un axil de compresión de $700 \mathrm{KN}$, el segundo tramo mantiene el valor del axil y, en el tercer tramo, se tiene una carga concentrada de valor $2.000 K N$ y una carga repartida de $60 \mathrm{KN} / \mathrm{m}$, simultáneamente con un axil de tracción de valor $200 \mathrm{KN}$. Finalmente, para el cálculo de la carga repartida de peso propio, se ha considerado un peso especifico para el material de la viga de valor $25 \mathrm{KN} / \mathrm{m}^{3}$

Para el cálculo se ha dividido cada tramo en dos elementos de manera que haya un nodo intermedio entre apoyos. En los dos primeros tramos el nodo se ha situado en el centro y en el tercero en el punto 
de actuación de la carga concentrada. Todas estas condiciones sobre la geometría, estado de carga, condiciones de los apoyos y discretización en elementos, se esquematizan en la figura 2(a)-(b).

La ecuación de los elementos a compresión en el primer tramo de viga es:

$$
A u^{(4)}+N u^{(2)}+k u=f
$$

donde la rigidez es $A=E I=119600 \mathrm{KNm}^{2}$, la carga axil de compresión $B=-N=-700 \mathrm{KN}$, el coeficiente $k=10000 \mathrm{KN} / \mathrm{m}^{2}$. Asimismo la ecuación de los elementos a compresión en el segundo tramo es:

$$
A u^{(4)}+N u^{(2)}=f
$$

donde se ha considerado, por el estado de carga, la hipótesis de no tener en cuenta la respuesta del terreno. La rigidez y la carga de compresión son las mismas que en el primer tramo y la carga repartida es únicamente aqui la debida al peso propio.

En el tramo tercero la ecuación diferencial es:

$$
A u^{(4)}-T u^{(2)}+k u=f
$$

$\operatorname{con} A=E I=490590 \mathrm{KNm}^{2}, B=T=200 \mathrm{KN}, k=10000 \mathrm{KN} / \mathrm{m}^{2}$.

Siguiendo el procedimiento indicado en el apartado 4.2 y su nota y en 5.1 , se determinan para cada elemento, empleando funciones de forma que son soluciones de la correspondiente ecuación diferencial homogénea, las matrices de rigidez y las ecuaciones de equilibrio de tipo local. Una vez realizado el ensamblado de dichas ecuaciones se obtiene la ecuación de equilibrio global y, tras la consideración de las condiciones de contorno que imponen los apoyos y resolver después el correspondiente sistema, se obtienen los desplazamientos en los nodos (movimientos verticales y giros) que se indican en la tabla de la figura 2(d). Entrando asimismo con los valores calculados, en la ecuación de equilibrio local de cada elemento, se determinan las acciones nodales de equilibrio, las cuales se indican en la tabla de la figura 2(c). Dichas acciones representan, en el extremo derecho de cada elemento, el valor del momento flector y del cortante, este último, modificado en el valor dado por $B u^{\prime}$ y los valores opuestos de dichos esfuerzos en el extremo izquierdo. Finalmente indicamos que la idea de acción equivalente, que permite aproximar con ventaja en este tipo de problemas, los desplazamientos, giros, flectores y cortantes, no la hemos desarrollado en la aplicación, por haber sido ésta expuesta, tal y como se ha comentado, de manera monográfica en [66] en un problema análogo sobre flexión de vigas.

\subsection{Aplicación a un problema de cálculo dinámico}

En este apartado aplicamos los resultados del punto 5 a la resolución de un problema particular de valor inicial consistente en la determinación de la respuesta de un sistema que se asimila a un oscilador simple sin amortiguamiento, constituido por una masa $m$, un resorte con constante de proporcionalidad $k$ y una acción que varia, de forma arbitraria,con el tiempo $f(t)$. En definitiva, se trata de obtener formalmente, a partir de la teoria de splines generalizados, un resultado equivalente, en la respuesta del sistema, al dado por la integral de Duhamel ([67, p. 16], [68, p. 101])] y, asimismo, destacar algunas consecuencias de la aplicación del concepto de acción equivalente, a un caso de tipo dinámico. Por otra parte, este problema de valor inicial, a pesar de su sencillez, es de gran interés en ingenieria, pues, como es bien conocido, cualquier sistema continuo, tras la discretización en el correspondiente sistema de $n$ grados de libertad y el posterior desacoplamiento de éste, conduce, para cada modo de vibración por separado, a la resolución de problemas como el planteado. Asimismo, el esquema de cálculo a seguir, por tratarse de un problema de valor inicial, sólo requiere la utilización del concepto de ecuación de equilibrio local, realizándose los cálculos, paso a paso, en cada intervalo de tiempo.

La ecuación diferencial que rige el sistema es

$$
L(u)=m u^{\prime \prime}+k u=f(t)
$$

donde $u(t)$ representa el desplazamiento. Un sistema fundamental de soluciones para $L(u)=0$ es $\{\cos (\omega t), \operatorname{sen}(\omega t)\}$ donde $\omega=\sqrt{k / m}$. Para un intervalo de tiempo genérico $[a, b]$ la de base de Lagrange, para las condiciones de interpolación dadas por $l_{1}(u)=u(a), l_{2}(u)=u(b)$, es $=$

$$
\left\{N_{1}(t)=\operatorname{sen}[\omega(b-t)] / \operatorname{sen}[\omega(b-a)] N_{2}(t)=\operatorname{sen}[\omega(t-a)] / \operatorname{sen}[\omega(b-a)]\right.
$$


$100 \mathrm{KN} / \mathrm{m}$

$60 \mathrm{KN} / \mathrm{m}$
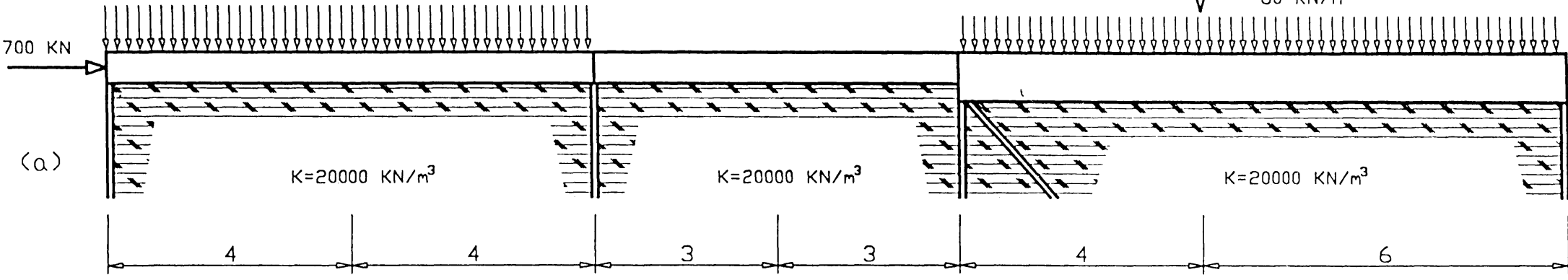

$\mathrm{K}=20000 \mathrm{KN} / \mathrm{m}^{3}$

$\mathrm{K}=20000 \mathrm{KN} / \mathrm{m}^{3}$
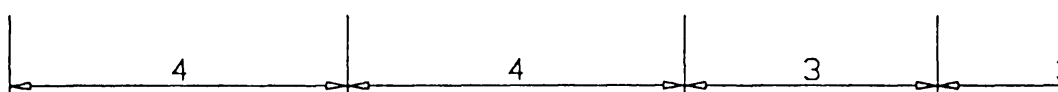

4

$0.5 \mathrm{~m}$

$0.5 m \square$

$0.8 \mathrm{~m} \underset{0.5 \mathrm{~m}}{\square}$

(b)

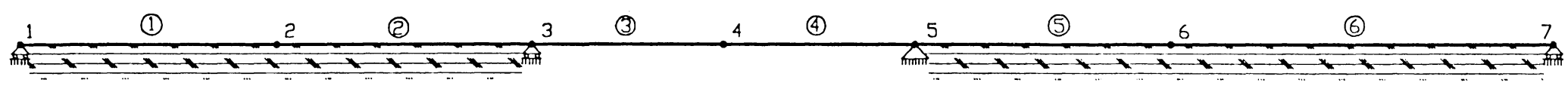

(c) ACCIONES NODALES DE EOUILIBRIO

\begin{tabular}{|c|c|c|c|c|}
\hline & \multicolumn{2}{|c|}{ FUERZA $(\mathrm{KN})$} & \multicolumn{2}{|c|}{ MOMENTO $\left(\mathrm{KN}^{*} \mathrm{M}\right)$} \\
\hline ELEMINODO & $1 z q$ & Der & Izq & Der \\
\hline 1 & 153.13 & 0.746 & 0.00 & 169.74 \\
\hline 2 & -0.746 & 156.30 & -169.74 & -9.05 \\
\hline 3 & -70.43 & 89.19 & 9.05 & -255.30 \\
\hline 4 & -89.19 & 107.95 & 255.30 & -544.20 \\
\hline 5 & 649.31 & -1054.47 & 544.20 & 2436.12 \\
\hline 6 & -945.53 & 195.12 & -2436.12 & 0.00 \\
\hline
\end{tabular}

Figura 2 (d) MOVIMIENTOS

\begin{tabular}{|c|c|c||}
\hline NODO & Y(M) & O(RAD). \\
\hline 1 & 0.00000 & -0.00442 \\
\hline 2 & -0.010380 & 0.00002 \\
\hline 3 & 0.00000 & 0.00433 \\
\hline 4 & 0.00973 & 0.00112 \\
\hline 5 & 0.00000 & -0.00882 \\
\hline 6 & -0.03003 & -0.00226 \\
\hline 7 & 0.00000 & 0.00772 \\
\hline
\end{tabular}


donde la longitud del intervalo debe ser tal que $\omega(b-a) \neq k \pi$ para $k$ entero. La ecuación de equilibrio local que verifican las soluciones de (6.3) es

$$
\frac{m \omega}{\operatorname{sen}[\omega(b-a)]}\left[\begin{array}{cc}
-\cos [\omega(b-a)] & 1 \\
1 & -\cos [\omega(b-a)]
\end{array}\right]\left[\begin{array}{l}
u(a) \\
u(b)
\end{array}\right]=\left[\begin{array}{l}
\int_{a}^{b} f N_{1} d t \\
\int_{a}^{b} f N_{2} d t
\end{array}\right]+\left[\begin{array}{c}
m u^{\prime}\left(a^{+}\right) \\
-m u^{\prime}\left(b^{-}\right)
\end{array}\right]
$$

tal y como se deduce de $\bar{l}_{1}(u)=m u^{\prime}\left(a^{+}\right), \bar{l}_{2}(u)=-m u^{\prime}\left(b^{-}\right)$, y de $k_{i j}=\bar{l}_{i}\left(N_{j}\right), i, j=1,2$.

En este caso, al tratarse de un problema de valor inicial, del conocimiento de $u(a), u^{\prime}\left(a^{+}\right)$pueden obtenerse los valores $u(b), u^{\prime}\left(b^{-}\right)$resultando las expresiones

$$
\begin{aligned}
& u(b)=\left(\cos [\omega(b-a)] \frac{\operatorname{sen}[\omega(b-a)]}{\omega}\right)\left[\begin{array}{c}
u(a) \\
u^{\prime}\left(a^{+}\right)
\end{array}\right]+\frac{1}{m \omega} \int_{a}^{b} f(t) \operatorname{sen}[\omega(b-t)] d t \\
& u^{\prime}\left(b^{-}\right)=\left(-\omega \operatorname{sen}[\omega(b-a)] \cos [\omega(b-a)]\left[\begin{array}{c}
u(a) \\
u^{\prime}\left(a^{+}\right)
\end{array}\right]+\frac{1}{m} \int_{a}^{b} f(t) \cos [\omega(b-t)] d t\right.
\end{aligned}
$$

Obsérvese cómo por esta via de los splines generalizados se obtiene para el intervalo $[a, b]=[a, T]$ la conocida integral de convolución o integral de Duhamel (segundo sumando de (6.4))

$$
\int_{t}^{T} f(t) \frac{\operatorname{sen}[\omega(T-t)]}{m \omega} d t
$$

la cual es obtenida usualmente a partir de la superposición de las respuestas de los sucesivos impulsos elementales en que se puede descomponer la acción $f(t)$, o también y de forma análoga, como la superposición de la respuestas a las funciones escalón.

Por otra parte, el concepto de acción equivalente dado en el apartado 5.2 permite aproximar la respuesta del sistema dentro del intervalo $[a, b]$ mediante la expresión interpoladora siguiente

donde

$$
U(t)=\hat{u} C \tilde{x}, \quad t \in[a, b]
$$

$$
\begin{aligned}
& \hat{u}=\left(u(a), u^{\prime}\left(a^{+}\right), u(b), u^{\prime}\left(b^{-}\right)\right) \\
& \tilde{x}^{t}=(\cos (\omega z), z \cos (\omega z), \operatorname{sen}(\omega z), z \operatorname{sen}(\omega z)), z=t-a
\end{aligned}
$$

y la matriz $C$ es

$$
C=\frac{1}{c_{3}^{2}-c_{1}^{2}}\left[\begin{array}{cccc}
c_{3}^{2}-c_{1}^{2} & -\omega\left(c_{1} c_{2}+c_{3}\right) & c_{1} c_{2}+c_{3} & -\omega c_{1}^{2} \\
0 & -c_{1}^{2} & l c_{3} & c_{1} c_{2}-c_{3} \\
0 & \omega\left(c_{1}+c_{2} c_{3}\right) & -\left(c_{2} c_{3}+c_{1}\right) & \omega c_{1} c_{3} \\
0 & -c_{1} c_{3} & l c_{1} & c_{2} c_{3}-c_{1}
\end{array}\right]
$$

con

$$
l=b-a, c_{1}=\operatorname{sen}(\omega l), c_{2}=\cos (\omega l), c_{3}=l \omega
$$

El producto de las matrices $C$ y $\tilde{x}$ dan como resultado un vector de funciones, las cuales constituyen una base de Lagrange para las condiciones de interpolación de Hermite definidas por $\hat{u}$. Asimismo, el conjunto de funciones dado en el vector $\tilde{x}$ son un sistema fundamental para el operador $L^{2}$. Obsérvese que la ecuación característica para el citado operador es $m^{2} r^{4}+2 \omega^{2} r^{2}+\omega^{4}=0$, la cual tiene por raíces $\omega i($ doble $),-\omega i($ doble $)$.

La respuesta aproximada $U(t)$ se torna en exacta cuando la acción está dada por una combinación lineal de las funciones $\cos (\omega t), \operatorname{sen}(\omega t)$, pues, en este caso, la proyección ortogonal en el subintervalo 
$[a, b]$, de $f(t)$ sobre el espacio engendrado por las funciones anteriores, coincide con la acción original.

La utilización de la expresión (6.6) permite reducir de manera notable el volumen de cálculos necesarios para la determinación de la respuesta del sistema. Este hecho se pone de manifiesto en el ejemplo numérico siguiente

Supongamos un sistema mecánico de masa $m=8 \times 10^{5} \mathrm{~kg}$ y constante de muelle del sistema de valor $k=10^{9} \mathrm{~N} / \mathrm{m}$. La frecuencia natural circular es $\omega=\sqrt{\mathrm{k} / \mathrm{m}}=35.35534 \mathrm{seg}^{-1}$ y el periodo natural $T=2 \pi / \omega=0.1777 \mathrm{seg}$. La acción $f(t)$ que hemos elegido es de tipo impulsivo y su valor, a lo largo del tiempo,se indica en el esquema de la figura $3(\mathrm{a})$. Se ha considerado para el cálculo un intervalo de tiempo de $0.40 \mathrm{seg}$ y condiciones iniciales nulas para el desplazamiento y la velocidad, determinándose mediante (6.4) y (6.5) la respuesta exacta en 91 instantes de tiempo que se corresponden con la división de los intervalos $[0.0,0.02][0.02,0.1][0.1,0.2][0.2,0.3]$ y $[0.3,0.4]$ en diez partes iguales para el primer intervalo y veinte partes iguales para los restantes. Se ha realizado, asimismo, una comparación entre dicha respuesta exacta $u(t)$ y dos respuestas aproximadas $U(t)_{I}, U(t)_{I I}$, que se han obtenido a partir del concepto de acción equivalente. En el primer caso, la respuesta aproximada $U(t)$, se ha obtenido interpolando con la expresión (6.6) los valores exactos de desplazamiento y velocidad en los extremos de los intervalos de tiempo citados. Como puede comprobarse en la gráfica de la figura 3 (b), a partir del instante $t=0.2 \mathrm{seg}$, donde el valor de la acción se hace nulo y las oscilaciones son libres, ambas respuestas coinciden. Consecuentemente, solamente se han tabulado los 51 valores que corresponden a instantes comprendidos entre el inicial y el de finalización de la acción exterior. El error cometido desde el instante inicial hasta dicho instante, $t=0.2 \mathrm{seg}$, se puede apreciar tanto en la gráfica como en la tabla. Por otra parte, considerando los subintervalos que tienen por extremos los instantes $t=0.0,0.02,0.06,0.1,0.15,0.2,0.4 \mathrm{seg}$, e interpolando nuevamente en los extremos de cada uno, obtenemos la respuesta que hemos denominado respuesta aproximada $U(t)_{I I}$, la cual,como puede apreciarse en la tabla, coincide prácticamente con la respuesta teórica (entre 0.2 y $0.4 \mathrm{seg}$ es igual a la respuesta exacta). De esta manera la respuesta aproximada $U(t)_{I I}$ obtenida por interpolación en seis intervalos, resulta, en este caso, equivalente a la exacta, calculada mediante las expresiones (6.4) y (6.5) en los 91 instantes citados.

\section{Comentarios finales y conclusiones}

Se ha concretado en este trabajo un método de análisis, construcción y cálculo de los splines generalizados que está basado en una interpretación matricial del planteamiento variacional de la teoría de splines. Dicho enfoque puede considerarse como alternativo a otras líneas de trabajo existentes que emplean esencialmente procedimientos algebraicos en la construcción y análisis de dichas funciones. La interpretación efectuada ha permitido, de manera natural, aplicar en el campo de los splines las técnicas matriciales utilizadas en el cálculo de estructuras y elementos finitos. El método que se ha propuesto para el cálculo de los splines es el de desplazamientos, aunque en el trabajo se comenta también, de forma esquemática, el método de compatibilidad. De acuerdo con los resultados obtenidos, tanto el enfoque seguido como la metodología desarrollada pueden considerarse originales en el área de los splines, pues en la extensa bibliografía consultada no se ha encontrado ningún desarrollo próximo al aqui elaborado.

Por otra parte, la linea de trabajo seguida ha permitido una cieria extensión del concepto de spline generalizado, modificando alguna de las condiciones en las que se viene basando la definición de dichas funciones: regularidad global en las funciones que definen el operador al que va asociado el spline, en lugar de la regularidad por subintervalos, como aqui se propone.

La formulación matricial se ha planteado a través de conceptos propios de la metodologia variacional aplicados a las funciones de forma locales que definen el spline, apareciendo, de manera directa, los elementos del cálculo matricial de estructuras: ecuación de equilibrio local, matriz de rigidez, vector de desplazamientos, vector de cargas nodales, etc., que encuentran sentido en el cálculo de splines con esta metodología. Asimismo, es posible trasladar al cálculo de estas funciones otros conceptos del cálculo matricial tales como expansión, ensamblado, condensación nodal, etc. Además, se ponerr de relieve ciertas ventajas o posibilidades que no se han explotado suficientemente en la práctica usual del cálculo de splines. 

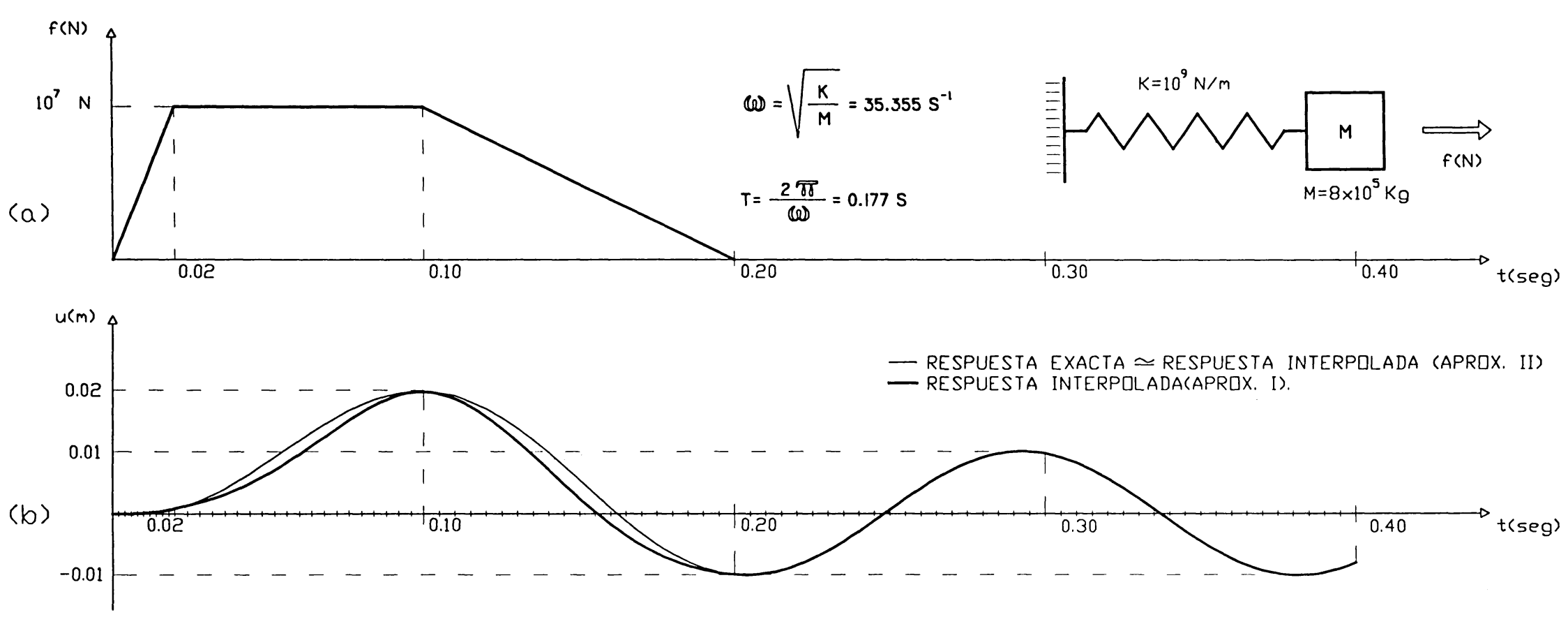

RESPUESTA

RESPUESTA

RESPUESTA

RESPUESTA

RESPUESTA

\begin{tabular}{|l|l|l|}
\hline EXACTA & APROX. 1 & APROX. I \\
\hline
\end{tabular}

\begin{tabular}{|c|c|c|c|}
\hline TSEG) & $U(T)($ OM $)$ & $U(T)_{1}\left(C_{M}\right)$ & $U(T)_{\|}\left(C_{H}\right)$ \\
\hline 0.000 & 0.0000 & 0.0000 & 0.0000 \\
\hline 0.002 & $0.833 E-4$ & $0.471 \mathrm{E}-3$ & $0.471 \mathrm{E}-3$ \\
\hline 0.004 & $0.666 E-3$ & $0.546 \mathrm{E}-3$ & $0.546 \mathrm{E}-3$ \\
\hline 0.006 & $0.224 \mathrm{E}-2$ & $0.203 E-2$ & $0.203 E-2$ \\
\hline 0.008 & $0.531 E-2$ & $0.502 E-2$ & $0.50 Z E-2$ \\
\hline 0.010 & 0.0104 & 0.0100 & 0.0100 \\
\hline 0.012 & 0.0178 & 0.0176 & 0.0176 \\
\hline 0.014 & 0.0282 & 0.0280 & 0.0280 \\
\hline 0.016 & 0.0420 & 0.0418 & 0.0418 \\
\hline 0.018 & 0.0595 & 0.0595 & 0.0595 \\
\hline 0.020 & 0.0813 & 0.0813 & 0.0813 \\
\hline
\end{tabular}

\begin{tabular}{|c|c|c|c|}
\hline & EXACTA & APROX. I & APROX. 11 \\
\hline$T(\operatorname{SEG})$ & $U_{T} X_{\left({ }_{(H H}\right)}$ & $\left(\mathrm{H}_{\mathrm{T}}\right)_{1}\left(\mathrm{O} H_{\mathrm{H}}\right)$ & $U(T)_{\|}(O M)$ \\
\hline 0.024 & 0.138 & 0.131 & 0.137 \\
\hline 0.028 & 0.212 & 0.185 & 0.208 \\
\hline 0.032 & 0.302 & 0.246 & 0.294 \\
\hline 0.036 & 0.406 & 0.316 & 0.396 \\
\hline 0.040 & 0.522 & 0.397 & 0.510 \\
\hline 0.044 & 0.647 & 0.488 & 0.636 \\
\hline 0.048 & 0.779 & 0.591 & 0.771 \\
\hline 0.052 & 0.916 & 0.706 & 0.911 \\
\hline 0.056 & 1.054 & 0.850 & 1.055 \\
\hline 0.060 & 1.192 & 0.962 & 1.192 \\
\hline 0.064 & 1.325 & 1.100 & 1.324 \\
\hline
\end{tabular}

\begin{tabular}{|l|l|l|}
\hline EXACTA & APROX. 1 & APROX. 11 \\
\hline
\end{tabular}

\begin{tabular}{|c|c|c|c|}
\hline$T(S E G)$ & UTTX(M) & $\left\langle(T)_{1}\left(O_{1}\right)\right.$ & $\left|U_{T}\right\rangle_{1}\left(C_{T}\right)$ \\
\hline 0.068 & 1.452 & 1.242 & 1.46 .7 \\
\hline 0.072 & 1.570 & 1.382 & 1.562 \\
\hline 0.076 & 1.677 & 1.518 & 1.666 \\
\hline 0.080 & 1.770 & 1.645 & 1.758 \\
\hline 0.084 & 1.847 & 1.756 & 1.837 \\
\hline 0.088 & 1.908 & 1.852 & 1.900 \\
\hline 0.092 & 1.951 & 1.923 & 1.946 \\
\hline 0.096 & 1.974 & 1.967 & 1.973 \\
\hline 0.100 & 1.978 & 1.970 & 1.976 \\
\hline 0.105 & 1.956 & 1.945 & 1.953 \\
\hline 0.110 & 1.902 & 1.863 & 1.893 \\
\hline
\end{tabular}

\begin{tabular}{|c|c|c|c|}
\hline & EXACTA & APROX. 1 & APROX. II \\
\hline$T(S E G)$ & $U_{T}(T(M)$ & $\left.u_{T}\right)_{1}\left(O_{1}\right)$ & $\left.\mid U_{T}\right)_{\|}\left(C_{M}\right)$ \\
\hline 0.115 & 1.817 & 1.737 & 1.801 \\
\hline 0.120 & 1.702 & 1.575 & 1.681 \\
\hline 0.125 & 1.559 & 1.384 & 1.537 \\
\hline 0.130 & 1.390 & 1.171 & 1.370 \\
\hline 0.135 & 1.200 & 0.943 & 1.185 \\
\hline 0.140 & 0.993 & 0.710 & 0.984 \\
\hline 0.145 & 0.773 & 0.476 & 0.771 \\
\hline 0.150 & 0.547 & 0.248 & 0.547 \\
\hline 0.155 & 0.319 & 0.0527 & 0.518 \\
\hline 0.160 & 0.0955 & -0.167 & 0.0922 \\
\hline 0.165 & -0.119 & -0.348 & -0.124 \\
\hline
\end{tabular}

\begin{tabular}{|l|l|l|}
\hline EXACTA & APROX. 1 & APROX. 11 \\
\hline
\end{tabular}

\begin{tabular}{|c|c|c|c|}
\hline TSEG) & U(TTXOM) & $\left(V_{T}\right)_{1}\left(O T_{1}\right)$ & $\left(U_{T}\right)_{\|}\left(C_{T}\right)$ \\
\hline 0.170 & -0.318 & -0.507 & -0.325 \\
\hline 0.175 & -0.499 & -0.643 & -0.506 \\
\hline 0.180 & -0.656 & -0.756 & -0.662 \\
\hline 0.185 & -0.786 & -0.847 & -0.791 \\
\hline 0.190 & -0.888 & -0.916 & -0.890 \\
\hline 0.195 & -0.958 & -0.965 & -0.959 \\
\hline 0.200 & -0.997 & -0.997 & -0.997 \\
\hline
\end{tabular}

Figura 3 
Asi, las ventajas del método propuesto se centran, fundamentalmente, en la posibilidad de manejar simultáneamente multitud de parámetros y diferentes condiciones de interpolación, es decir, modificar a voluntad, en cada subintervalo, el valor de las funciones que definen el operador diferencial al que va asociado el spline y asimismo utilizar condiciones de interpolación que pueden ser diferentes de unos nodos a otros, teniendo la opción de modificar éstas en cualquier momento, sin necesidad de cambiar la ecuación que hemos denominado de equilibrio global.

Por otro lado, también se ha incluido la posibilidad de incorporar al cálculo las acciones que hemos denominado de tipo puntual. Éstas, como se comenta en el trabajo, han sido empleadas por algunos autores de una manera indirecta al considerar la inclusión de nodos adicionales, fijando adecuadamente sus ordenadas para evitar, por ejemplo, la aparición de puntos de inflexión extraños. También se han empleado en los splines de suavizado donde la curva spline se une, de manera elástica, a ciertos puntos de ordenada fija. Para dichos casos la construcción se ha efectuado para tipos muy particulares de condiciones de interpolación y de una manera un tanto rigida, que contrasta con la versatilidad del enfoque aqui desarrollado.

Por lo que se refiere a otros aspectos, puede señalarse el resultado descrito para el caso de operadores autoadjuntos en la forma usual, que relaciona la unicidad de solución del problema de interpolación con splines generalizados, con la unicidad de solución de un problema de interpolación polinomial de HermiteBirkoff. También destacamos el resultado de reciprocidad en teoria de splines, lo que pone de manifiesto el alcance de la generalización realizada. Esta línea permitiria dar interpretación, en el campo de los splines, a otros teoremas distintos al de reciprocidad, como son, por ejemplo, los de Castigliano, trabajo minimo, etc.

La teoria de splines generalizados como técnica de interpolación encuentra un importante campo de aplicación en la aproximación de las soluciones de problemas de contorno unidimensionales cuando se emplean las técnicas de elementos finitos o elementos de contorno. En esta linea el enfoque seguido ha permitido demostrar e interpretar con facilidad en que casos la solución de estos problemas de contorno es nodalmente exacta. Aunque lo anterior es bien conocido por la teoría de elementos de contorno y de la función de Green y, especialmente, desde el trabajo de P. Tong ([22]), el planteamiento desarrollado, además de lo indicado anteriormente, nos ha conducido a la idea de acción equivalente, generalizando la idea de acción nodal equivalente. Hay que señalar también como resultado, derivado del planteamiento propuesto, que se obtienen soluciones nodalmente exactas, independientemente de que las funciones de prueba utilizadas sean,o no, splines generalizados; bastando con que éstas intervengan solamente como funciones de punderación. Además, la exactitud nodal de la solución se puede conseguir incluso para derivadas altas a partir de las ecuaciones de equilibrio de los elementos.

Se indica finalmente que las ideas expuestas en el trabajo, se han ilustrado con aplicaciones a casos sencillos, en diferentes contextos, como son,por ejemplo, el de las técnicas gráficas, el propio del cálculo de estructuras y el del cálculo dinámico. Esto viene a poner de manifiesto que la potencialidad y versatilidad de los planteamientos matriciales de la teoría de splines generalizados permite englobar, dentro de una misma metodologia,áreas que tradicionalmente son estudiadas de manera separada.

\section{REFERENCIAS}

[1] AHLBERG, J. H., NILSON, E. N. y WALSH, J. L.: The Theory of Splines and Their Applications, Academic Press, New York, 1967.

[2] SCHULTZ, M. H.: Spline Analysis, Prentice-Hall, Englewood Cliffs, New Jersey, 1973.

[3] SCHUMAKER, L. L.: Spline Functions: Basic Theory, John Wiley \& Sons, New York, 1981

[4] GREVILLE, T. N. E.: Theory and Applications of Spline Functions, Academic Press, New York, 1969.

[5] PRENTER, P. M.: Splines and Variational Methods, John Wiley \& Sons, New York, 1975.

[6] de BOOR, C.: A Practical Guide to Spline, Springer-Verlag, New York, 1978.

[7] SPÄTH, H.: One Dimensional Spline Interpolation Algorithms, A K Peters Wellesley, MA,1995.

[8] DIERCKX, P.: Curve and Surface Fitting with Splines, Clarendon Press, Oxford, 1993.

[9] QIULIN, D. y DAVIES, B. J.: Surface Engineering Geometry for Computer-Aided Design and Manufacture, Ellis Horwood Limited,Chichester, 1987. 
[10] YAMAGUCHI, F.: Curves and Surface in Computer Aided Geometric Design, Springer-Verlag, Berlin Heidelberg, 1988.

[11] FARIN, G.: Curves and Surface for Computer Aided Geometric Design, A Practical Guide, Academic Press, Inc., New York, 1988.

[12] ROGERS, D. F. y ADAMS, J. A.: Mathematical Elements for Computer Graphics, McGraw-Hill, New York, 1990.

[13] MORTENSON, M. E.: Geometrics Modeling, John Wiley and Sons, New York, 1985.

[14] BÖHN, W., FARIN, G. y KAHMANN, J.: "A survey of curve and surface methods in CAGD", Computer Aided Geometric Design, 1 pp. 1-60, North-Holland, 1984.

[15] BARTELS, R. H., BEATTY, R. H. y BARSKY, B. A.: An Introduction to Spline for use in Computer Graphics and Geometric Modeling, Morgan Kaufmann Publishers, Inc., U.S.A., 1987.

[16] CHEUNG, Y. K., FAN, S. C. y WU, C. Q.: "Spline finite strip in structural analysis", Proc. Int. Conf. on Finite Element Method, Shangai, pp. 704-709, 1982.

[17] THAM, L. G., LI, W. Y. y CHEUNG, Y. K.: "Bending of skew plates by spline-finite-strip method", Computers \& Structures, Vol. 22, No. 1, pp. 31-38, 1986.

[18] LEUNG, A. Y. T. y AU, F. T.: "Spline finite element for bean and plate", Computers \& Structures, Vol. 37, No. 5, pp. 717-729, 1990

[19] LUAH, M. H. y FAN, S. C.: "Analysis of complicated plates by a nine-node spline plate element", Computers \& Structures, Vol. 45, No. 2, pp. 243-252, 1992.

[20] AU, F. T. K. y CHEUNG, Y. K.: "Isoparametric spline finite strip for plane structures", Computers \& Structures, Vol. 48, No. 1, pp. 23-32, 1993.

[21] TIMOSHENKO, S. P.: History of Strength of Materials, Dover Publ. Inc., New York, 1983.

[22] TONG, P.: "Exact solution of certain problems by finite-element method", AlAA Journal, Vol. 7, No. 1, pp. 178-180, 1969.

[23] HERRERA, I.: Boundary Methods: An Algebraic Theory, New York, Pitman, 1984.

[24] JEROME, J. W.: "Linear self-adjoint multipoint boundary value problem", Numer. Math., 15, pp. 433$449,1970$.

[25] de BOOR, C. y LYNCH, R. E.: "On splines and their minimun properties", Journal of Mathematics and Mechanics, Vol. 15, No. 6, pp. 953-969, 1966.

[26] JEROME, J. W. y VARGA, R. S.: "Generalizations of spline functions and applications to nonlinear boundary value and eigenvalue problems", en [4].

[27] ROMERO, J. L., MORANT, J. L. y CORRALES, J. M.: "Operadores de relajación en espacios de Hermite $H^{(2)}(\Delta)$. Aplicación al cálculo de splines", en / Congreso de Métodos Numéricos en Ingenieria, SEMNI, pp. 228-232, Las Palmas, 1990.

[28] ROMERO, J. L., GARCIAA, F. y MORANT, J. L.: "Calculation of splines by iterative methods based on relaxation", en Proceeding of International Simposium of Numerical Analysis (ISNA '92), pp. 229-245, Prague, Czechoslovakia, 1992.

[29] ROMERO, J. L., CORRALES, J. M. y GARCÍA, F.: "Interpolación mediante splines generalizados del tipo $a(x) D^{m}$ - splines, en // Congreso de Métodos Numéricos en Ingenieria, SEMNI, pp.720-730, La Coruña, 1993.

[30] INCE, E. L.: Ordinary Differential Equations, Dover Publ. Inc., New York, 1956.

[31] STAKGOLD, I.: Green's Functions and Boundary Value Problems, John Wiley \& Sons, New 
[32] STRANG, G. y FIX, J.: An Analysis of the Finite Element Method, Prentice-Hall,Inc., Englewood Cliffs, N. J., 1973.

[33] KYTE, P. K.: An Introduction to Boundary Element Methods, CRC Press, Inc., U.S.A.,1995.

[34] PILKEY, W. D. y WUNDERLICH, W.: Mechanics of Structures, Variational and Computational Methods, CRC Press, Inc., U.S.A. 1994.

[35] SPÄTH, H.: "Interpolation by certain quintic splines", Computer J. 12, pp, 292-293, 1969.

[36 ] MUND, E. H., HALLET, P. y HENNART, J. P.: "An algorithm for interpolation of functions using quintic splines", J. Comput. Appl. Math, 1, pp. 279-288, 1975.

[37] GREVILLE, T. N. E.: " Interpolation by generalized spline functions", MRC, Technical Summary Report \# 476, Madison, Wisconsin, 1964.

[38] SCHWEIKERT, D. G.: "An interpolation curva using a spline in tension", J. Math. \& Physics, Vol. 45, pp. 312-317, 1966.

[39] NIELSON, G. M.: "Some piecewise polynomial alternatives to splines under tension". Computer Aided Geometric Design, pp. 209-235, 19:4.

[40] SALKAUSKAS, K.: "C 1 splines for interpolation of rapidly varying data", Journal of Mathematics, Vol. 14, Number 1, pp. 239-250, Winter 1974

[41] PILCHÉR, D.: " Smooth parametric surfaces", en Computer Aided Geometric Design, BarnhillRiesenfeld (eds.), Academic Press, New York, 1974.

[42] CLINE, A. K.: "Scalar and planar value curve fitting using splines under tension", Numerical Mathematic Comm. ACM, Vol. 17, Number 4, pp. 218-220, 1974.

[43] BARSKY, B. A.: "Exponential and polynomial methods for applying tension to interpolating spline curve". Computer Vision, Graphics and Image Processing, 27, pp. 1-18, 1984.

[44] FOLEY, A. T.: "Local control of interval tension using weighted splines", Computer Aided Geometric Design, 3, pp. 281-294, 1986.

[45] FOLEY, A. T.: "Interpolation with interval and point tension control using cubic weighted v-splines", ACM Transactions on Mathematical Software, Vol. 13, No. 1, pp. 68-96, 1987.

[46] HAGEN, H. y SCHULZE, G.: "Variational principles in curve and surface design", en Geometric Modeling, Springer-Verlag, pp. 161-184,1991.

[47] VLASOV, V. Z. y LEON`EV, U. N.: Beams, Plates and Shells on Elastic Foundations, Israel Program for Scientific Traslations, Jerusalen (traslated from Russian), 1966.

[48] MIRANDA, C. y NAIR, K.: "Finite beam on elastic foundation", Journal of the Structural Division Proceedings of the American Society of Civil Engineers, pp. 131-142,1966.

[49] TIN, B.: "Finite beam on elastic foundation with restraints", Journal of Structural Division ASCE, pp. 611-621, 1982.

[50] TIN, B. y MOCKRY, E. F.: "Beam on elastic foundation finite element", Journal of Structural Engineering, Vol. 110, No. 10, pp. 2324-2339, 1984.

[51] MARTÍNEZ, M.: "Análisis de la viga simple sobre lecho elástico a partir del método de los elementos de contorno", Hormigón y Acero, Madrid, 1989.

[52] SENDOV, B. y ANDREEV, A.: "Approximation and interpolation theory", en Handbook of Numerical Analysis, Vol. III, (CIARLET, P. G. y LIONS, J. L., Editors), North-Holland, 1994.

[53] VARGA, R. S.: Functional Analysis and Approximation Theory in Numerical Analysis, Society for Industrial and Applied Mathematics, Philadelphia, Pennsylvania, 1971.

[54] ADAMS, R. A.: Sobolev Spaces, Academic Press, New York, 1975. 
[55] LARKIN, F. M.: "An interpolation procedure based on fitting elasticas to given data points", Culham Operating System, Note 5/66, Theory Division, Culham Laboratory, 1966.

[56] GLASS, J. M.: "Smooth-curve interpolation: a generalized spline-fit procedure", BIT, 6, pp. 277-293, 1966.

[57] WOODFORD, C. H.: "Smooth curve interpolation", BIT, 9, pp. 69-77, 1969.

[58] MALCOLM, M. A.: "On the computation of nonlinear spline functions", SIAM J. Numer. Anal., Vol. 14, No. 2,1977.

[59] GARDAN, Y.: Numerical Methods for CAD, Vol. I, Hermes Publishing, London, 1985.

[60] PRZEMIENIECKI, J. S.: Theory of Matrix Structural Analysis, Dover, New York, 1968.

[61] ODEN, J. T. y CAREY, G.: Finite Elements, Mathematical Aspects, Vol. 4, Prentice-Hall, Inc. E. C., 1983.

[62] ZIENKIEWICZ, O. C. y TAYLOR, R. L.: El Método de los Elementos Finitos, Vol. 1 McGraw-Hill, 1994

[63] CARDONA, J. y SAMARTIN, A.: "Aplicación del método de los elementos finitos a problemas de interpolación", Revista Intemacional para Cálculo y Diseño en Ingeniería, Vol. 2, 2, pp.165-194, 1986.

[64] STOER, J.: "Curve fitting with clothoidal splines", J. Res. Nat. Bur. Standards, Vol. 87, No. 4, pp. 317346, 1982.

[65] MEEK, D. S. y WALTON, D. J.: "The use of Cornu spirals in drawing planar curves of controlled curvature", Joumal of Computational and Applied Mathematics, 25, pp. 69-78, 1989.

[66] ROMERO, J. L. y ORTEGA, M. A.: "Acciones equivalentes y solución en desplazamientos interpolada en la viga de Bernoulli-Euler", Informes de la Construcción, Vol. 49. $N^{0} 454$, pp. 5-27,1998.

[67] MEIROVITCH, L.: Analytical Methods in Vibrations, Macmillan Publishing Co., Inc., New York, 1967.

[68] CLOUGH, R. W. y PENZIEN, J.: Dinamic of Structures, Mc Graw-Hill, Inc., 1975. 